\title{
Intelligent Monitoring System With High Temperature Distributed Fiberoptic Sensor For Power Plant Combustion Processes
}

\author{
FINAL TECHNICAL REPORT
}

Submitted to

U. S. Department of Energy

Performance Period: 09/27/2002 to 09/26/2006

Authors: Kwang Y. Lee, Stuart S. Yin, and Andre Boehman

The Pennsylvania State University Department of Electrical Engineering University Park, PA 16802

Ph. (814) 865-2621, Fax (814) 865-7065

kwanglee a.psu.edu

DOE Award Number: DE-FG26-02NT41532 


\section{DISCLAIMER}

"This report was prepared as an account of work sponsored by an agency of the United States Government. Neither the United States Government nor any agency thereof, nor any of their employees, makes any warranty, express or implied, or assumes any legal liability or responsibility for the accuracy, completeness, or usefulness of any information, apparatus, product, or process disclosed, or presents that its use would not infringe privately owned rights. Reference herein to any specific commercial product, process, or service by trade name, trademark, manufacturer, or otherwise does not necessarily constitute or imply its endorsement, recommendation, or favoring by the United States Government or any agency thereof. The views and opinions of authors expressed herein do not necessarily state or reflect those of the united States Government or any agency thereof." 


\begin{abstract}
The objective of the proposed work is to develop an intelligent distributed fiber optical sensor system for real-time monitoring of high temperature in a boiler furnace in power plants. Of particular interest is the estimation of spatial and temporal distributions of high temperatures within a boiler furnace, which will be essential in assessing and controlling the mechanisms that form and remove pollutants at the source, such as NOx. The basic approach in developing the proposed sensor system is three fold: (1) development of high temperature distributed fiber optical sensor capable of measuring temperatures greater than $2000 \mathrm{C}$ degree with spatial resolution of less than $1 \mathrm{~cm}$; (2) development of distributed parameter system (DPS) models to map the three-dimensional (3D) temperature distribution for the furnace; and (3) development of an intelligent monitoring system for real-time monitoring of the $3 \mathrm{D}$ boiler temperature distribution.

Under Task 1, we have set up a dedicated high power, ultrafast laser system for fabricating infiber gratings in harsh environment optical fibers, successfully fabricated gratings in single crystal sapphire fibers by the high power laser system, and developed highly sensitive long period gratings $(\mathrm{lpg})$ by electric arc. Under Task 2, relevant mathematical modeling studies of NOx formation in practical combustors have been completed. Studies show that in boiler systems with no swirl, the distributed temperature sensor may provide information sufficient to predict trends of NOx at the boiler exit. Under Task 3, we have investigated a mathematical approach to extrapolation of the temperature distribution within a power plant boiler facility, using a combination of a modified neural network architecture and semigroup theory. Given a set of empirical data with no analytic expression, we first developed an analytic description and then extended that model along a single axis.
\end{abstract}


TABLE OF CONTENTS

FINAL TECHNICAL REPORT

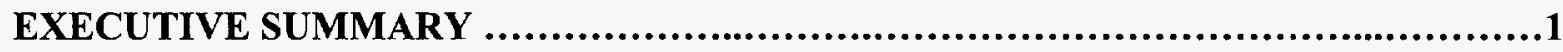

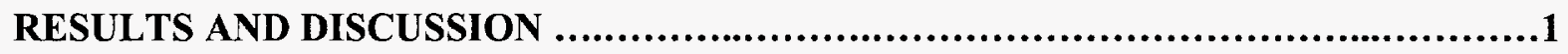

TASK 1. FIBEROPTIC SENSOR DEVELOPMENT ..................................

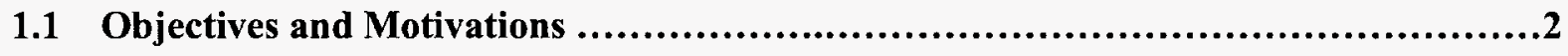

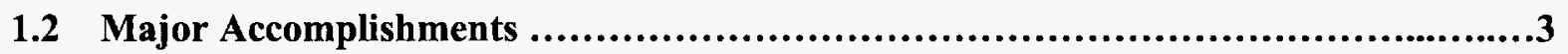

1.2.1 Demonstrate the effect of micro-machined gratings on single crystal sapphire

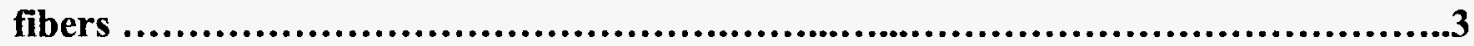

1.2.2 Demonstrate the possibility of using super continuum highly broad band

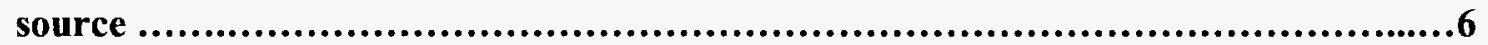

1.2.3 Investigate the more accurate patterning and micro-structuring method than mechanical dicing.....................................................................................8

1.2.4 Successfully fabricationg in-fiber gratings in harsh environment optical fibers by high

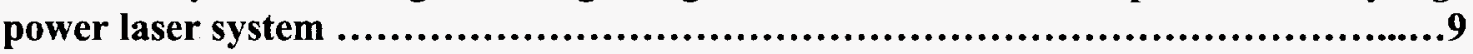

1.2.5 Perform temperature test with femostosecond laser inscribed FBG in high temperature furnace ....................................................................11

1.2.6 Demonstrate stable performance of our high temperature sensor in Mckenna Burner ..................................................................................13

1.2.7 Dmonstrate good long term stability of the femtosecond laser inscribed FBG sensor ..................................................................................16

1.2.8 Successfully demonstrate multi-parameter sensing capability ...................................18

1.2.9 Perform high temperature sensing using sapphire Bragg grating fabricated by femostosecond laser irradiation .............................................................20

1.2.10 Develop highly sensitive long period gratings (LPG) by electric arc ...................22

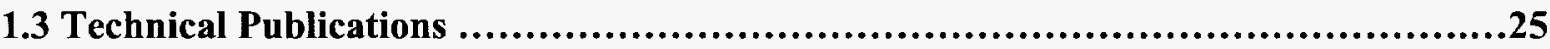

TASK 2: BOILER FURNACE MONITORING MODEL DEVELOPMENT ...............10

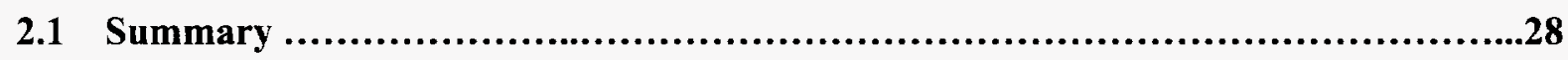

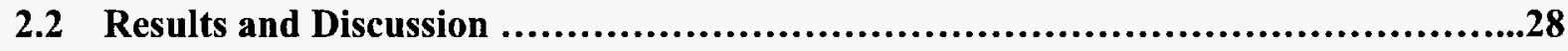

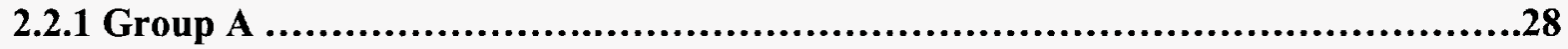

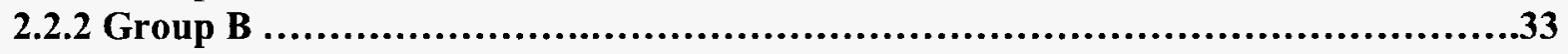

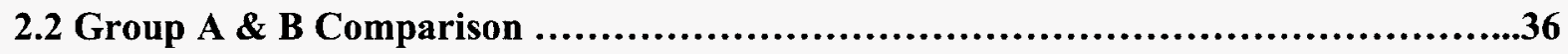


TABLE OF CONTENTS (Continued)

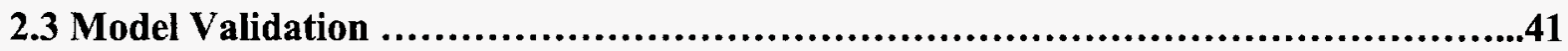

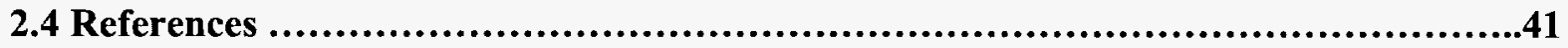

TASK 3. INTELLIGENT MONITORING SYSTEM DEVELOPMENT ....................42

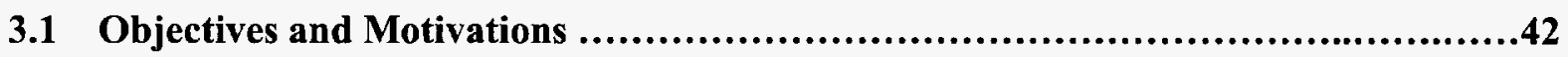

3.2 Development of Intelligent Monitoring System .....................................43

3.2.1 Monitoring of Temperature Distribution in Boiler Furnace .............................43

3.2.2 Extrapolation of Enthalpy in a Power Plant ...........................................44

3.2.3 Extrapolation of Transient Heat Transfer ..............................................44

3.2.4 Failures/shortcomings of Conventional ANN's ....................................50

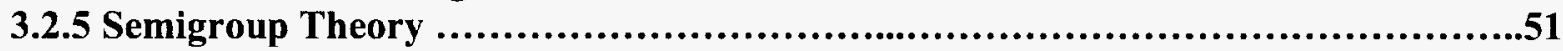

3.2.6 Relationship of Semigroup Theory to Neural Network Design........................52

3.2.7 Proposed Neural Netowork Archtecture ................................................53

3.2.8 Learning Algorithm of Proposed System-type NN .......................................54

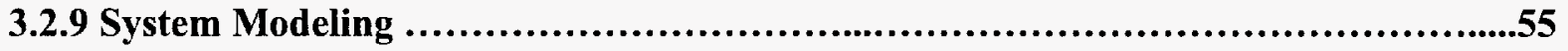

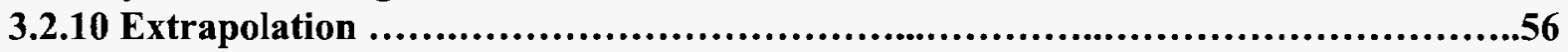

3.3 Simulation and Estimation Results ...................................................56

3.3.1 Monitoring of Temperature Distribution in Boiler Furnace .............................56

3.3.2 Demonstration of Extrapolation Capability for Enthalpy in a Power Plant ...........60

3.3.2 Demonstration of Extrapolation Capability for Transient Heat Transfer...............64

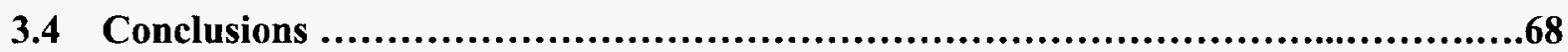

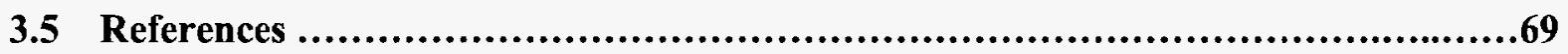

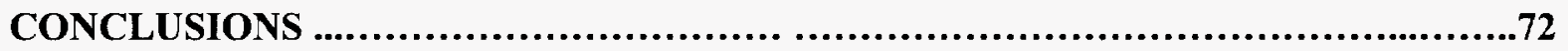

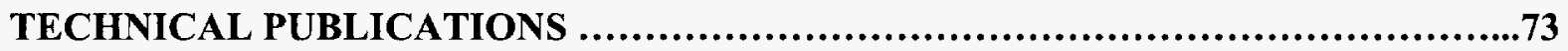

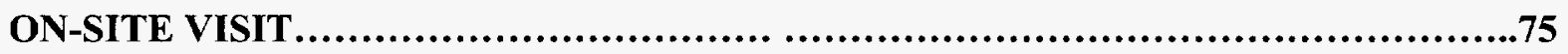

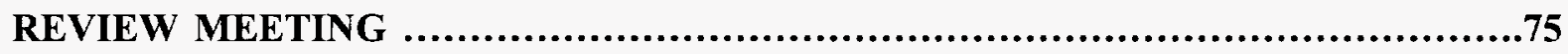




\title{
FINAL TECHNICAL REPORT
}

\author{
September 26, 2006
}

Title: Intelligent Monitoring System With High Temperature Distributed Fiberoptic Sensor For Power Plant Combustion Processes

Authors: $\quad$ Kwang Y. Lee, Stuart S. Yin, and Andre Bochman

Students: J.A. Chavez, S.H. Nam, C. Zhan, Melanie Fox, B.H. Kim, John

Valas

The Pennsylvania State University

Department of Electrical Engineering

University Park, PA 16802

Ph. (814) 865-2621, Fax (814) 865-7065

kwanglee@psu.edu

Grant Number: DE-FG26-02NT41532

Performance Period: 09/27/2002 to 09/26/2006

\section{EXECUTIVE SUMMARY}

The objective of the proposed work is to develop an intelligent distributed fiber optical sensor system for real-time monitoring of high temperature in a boiler furnace in power plants. Of particular interest is the estimation of spatial and temporal distributions of high temperatures within a boiler furnace, which will be essential in assessing and controlling the mechanisms that form and remove pollutants at the source, such as NOx.

The basic approach in developing the proposed sensor system is three fold: (1) development of high temperature distributed fiber optical sensor capable of measuring temperatures greater than $2000 \mathrm{C}$ degree with spatial resolution of less than $1 \mathrm{~cm}$; (2) development of distributed parameter system (DPS) models to map the three-dimensional (3D) temperature distribution for the furnace; and (3) development of an intelligent monitoring system for real-time monitoring of the $3 \mathrm{D}$ boiler temperature distribution.

Under Task 1, we have set up a dedicated high power, ultrafast laser system for fabricating in-fiber gratings in harsh environment optical fibers, successfully fabricated gratings in single crystal sapphire fibers by the high power laser system, and developed highly sensitive long period gratings $(\mathrm{lpg})$ by electric arc. Under Task 2 , relevant mathematical modeling studies of NOx formation in practical combustors have been completed. Studies show that in boiler systems with no swirl, the distributed temperature sensor may provide information sufficient to predict trends of NOx at the boiler exit. Under Task 3, we have investigated a mathematical approach to extrapolation of the temperature distribution within a power plant boiler facility, using a combination of a modified neural network architecture and semigroup theory. Given a set of empirical data with no analytic expression, we first developed an analytic description and then extended that model along a single axis. 


\section{RESULTS AND DISCUSSION}

\section{TASK 1. FIBEROPTIC SENSOR DEVELOPMENT}

\subsection{Objectives and Motivations}

The objective of this task is to develop an innovative high temperature distributed fiber optic sensor to precisely monitor the temperature distribution inside a boiler, which, in turn, could substantially increase the burning efficiency and reduce the pollution emission (e.g., $\mathrm{NO}_{\mathrm{x}}$ ). Figures 1.1(a) and 1.1(b) illustrate a power plant and a boiler with embedded fiber optic sensors.

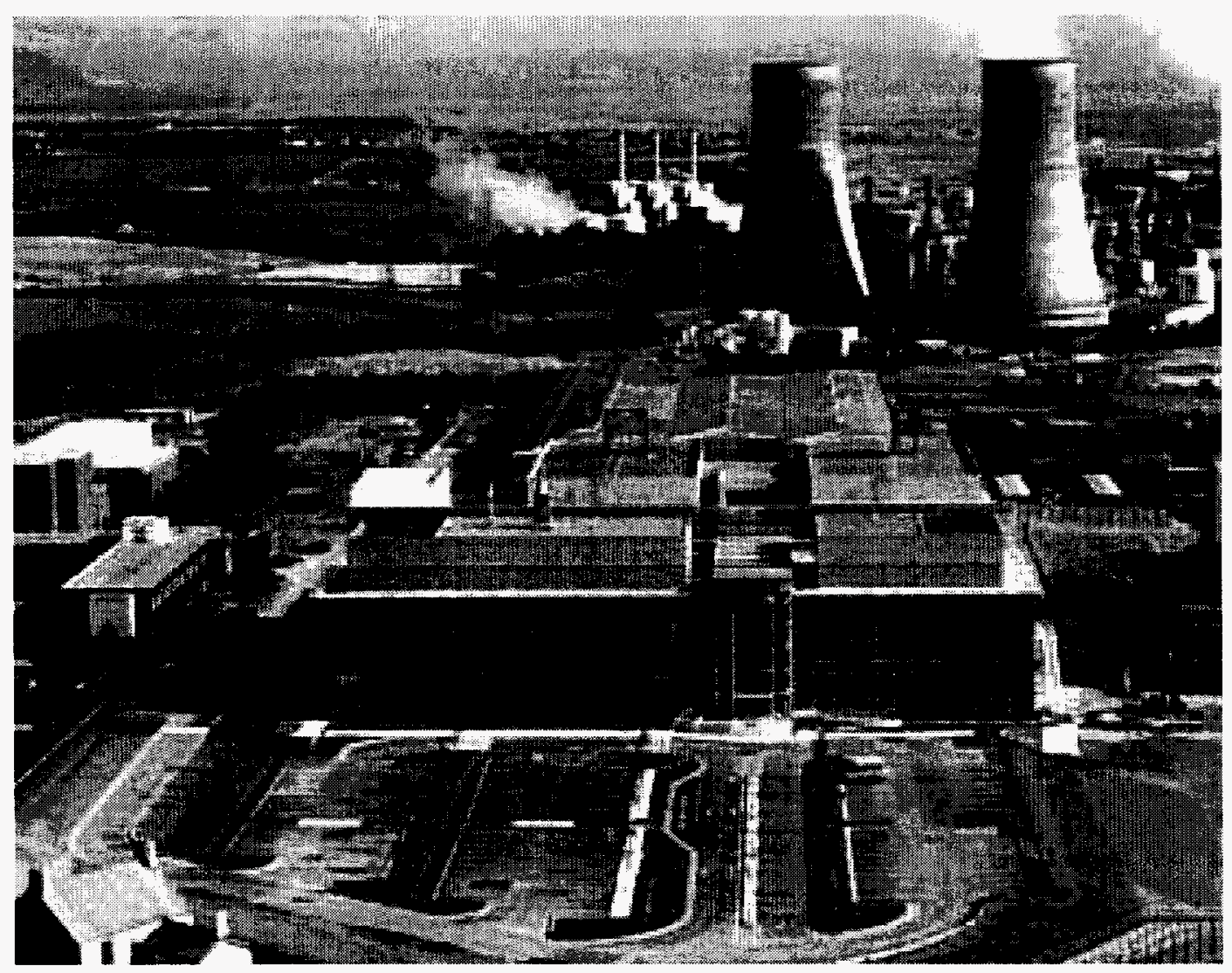

Fig. 1.1(a) A picture of a power plant 


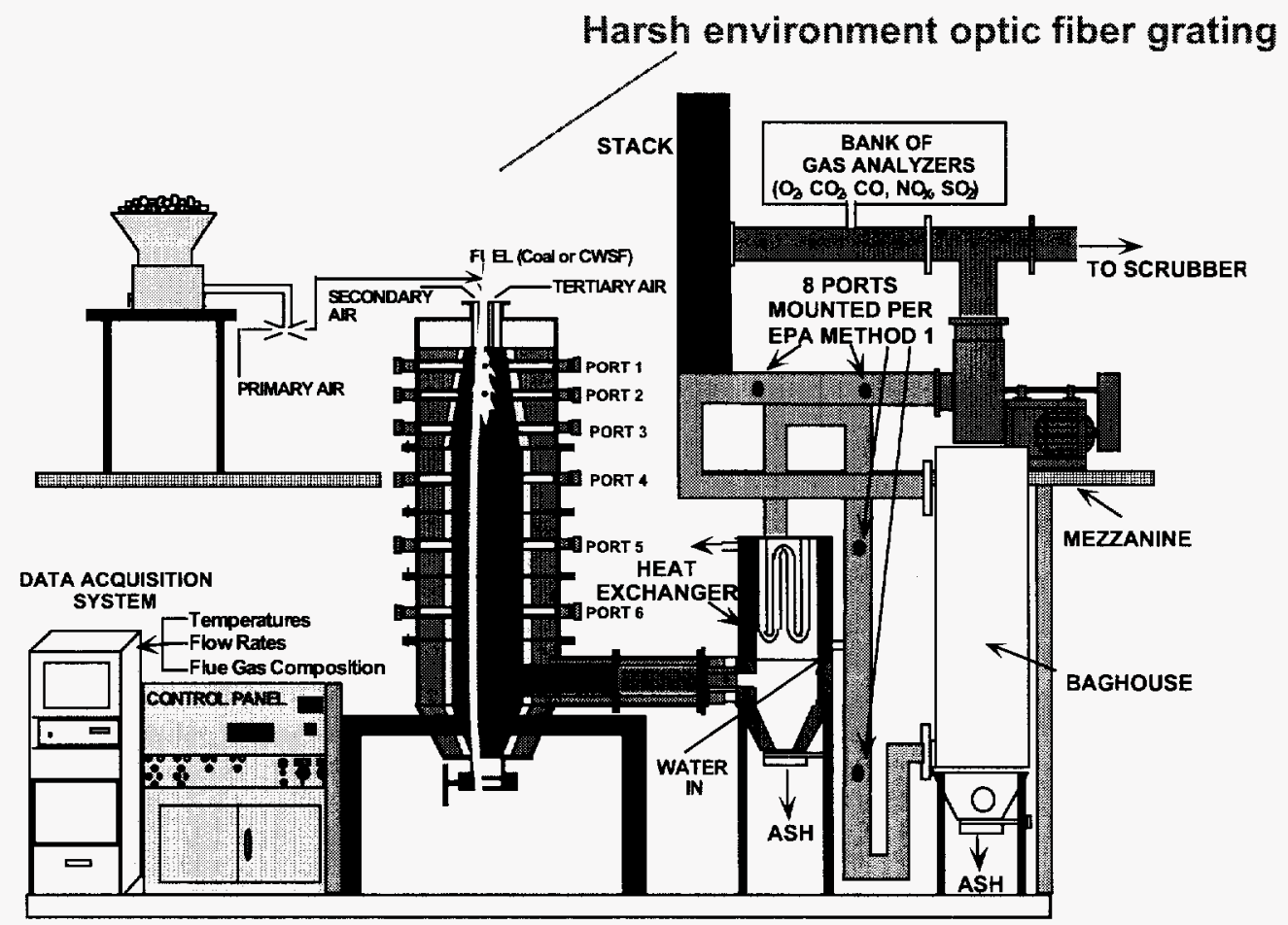

Fig. 1.1 (b) A boiler with embedded fiber optic sensor.

\subsection{Major Accomplishments:}

\subsubsection{Demonstrate the effect of micro-machined gratings on single crystal sapphire fibers.}

We showed that the micro-structuring is possible either by mechanical dicing or chemical etching after the patterning by lithography in the last year report. To demonstrate the effect of micro-machined gratings, we conducted following experiments.

- To fabricate in-fiber grating with large depth, we used mechanical dicing approach. In the experiment, a $5 \mathrm{~cm}$ long single crystal sapphire fiber, with 250 micron diameter, was used. In order to hold the fiber, the fiber sample was attached to a 2" x 2" glass substrate with the use of Crystalbond 509 adhesive and a hot plate. The glass substrate was then placed on a computer-controlled chuck underneath a diamond saw blade. The substrate was firmly held by the vacuum chuck once it was properly aligned. The blade width was 40 micron and the grating pitch was 
100 micron. The number of the notches was one hundred. The depth of the grating was 30 micron. After finishing the dicing, the glass substrate was heated on the hot plate and the sapphire fiber samples were carefully taken out of the substrate and cleaned with acetone.

- Figure 1.2 shows the fabricated grating using dicing approach. As we can see from the figure, overall structures were well formed but the edges of the notches were not smooth. This is the limitation of dicing approach. But it provides simple and fast means for micro-machining.

- To see the effect of the micro-machined fiber, we built the experimental setup as shown in Fig. 1.3. The light source was HP 8168E tunable laser. As the output of the tunable laser was connectorized with $\mathrm{FC} / \mathrm{PC}$, collimating optics was used to collimate the beam coming out of the connector end. NEW FOCUS model 9091 five-axis fiber aligner was used for this purpose. The collimated light beam was focused on the one end of the sapphire fiber sample by a microscope object lens (x20). The output beam of the sapphire fiber was directly coupled to a regular multimode fiber which was connected to HP 70951B optical spectrum analyzer (OSA). To get the optimum beam coupling between the fibers, Newport 462 series precise 3 axis aligner was used. A sample chamber was made to contain the index matching oil for the cladding of the sapphire fiber. The fiber sample penetrated this chamber and the index matching oil was provided through top open cover.

- For the micro-machined gratings to work as long period gratings (LPG), well defined uniform cladding layer has to be formed. Circumventing this problem, one of the ways for watching the effect of micro-machined gratings is to observe radiation coupling assisted by the gratings. A simple slab waveguide model can be used to explain this effect. If the periodic perturbation is to couple the light from the guided mode to a wave propagating into the surrounding cladding and making angle $\theta$ with the direction of propagation as shown in Fig 4(a), then we must have the following relation as shown in Fig. 1.4(b).

$$
\beta-K=k_{0} n_{s} \cos \theta
$$

where $\beta=2 \pi n_{e f f} / \lambda$ (propagation constant), $K=2 \pi / \Lambda$ (grating vector)

When this, so called, quasi-phase matching condition is satisfied, the radiation coupling from the guide mode to cladding occurs. Fig 1.4(c) shows the calculated wavelength dependence of radiation angle according to the quasi-phase matching condition. In the calculation, the effective refractive index of core is assumed to 1.78. The region below $0^{\circ}$ angle means that no radiation coupling is allowed. As we can see from the graph, when the refractive index of the cladding is 1.765 , the radiation coupling occurs for the longer wavelength than $1550 \mathrm{~nm}$. This model gives us good qualitative explanation for the case of sapphire fiber. For a certain refractive index of cladding, the radiation coupling will occur for longer wavelength than a specific value. 


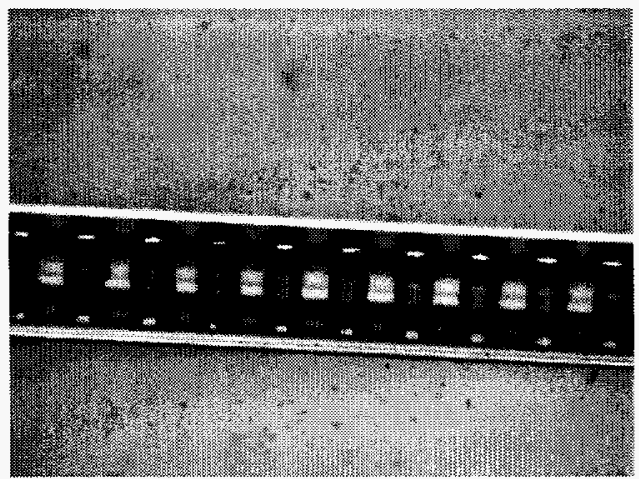

Fig. 1.2 Fabricate in-fiber long period grating in single crystal sapphire fiber by precise dicing.

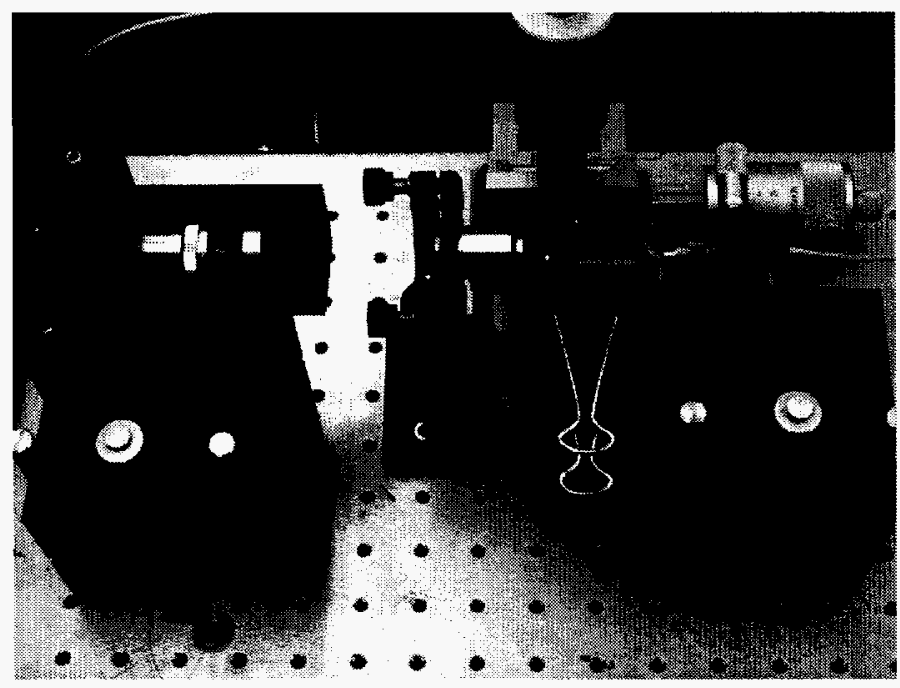

Fig 1.3. The experimental setup for measuring radiation-mode coupling effect by microstructured gratings in the sapphire fiber

- While changing the refractive index matching oil (Cargille Laboratories, refractive index liquids $\mathrm{M}$ series) for the cladding of the fiber in the chamber, output spectrums were observed. The measured spectrums are shown in Fig. 1.4. With air cladding and 1.79 index cladding, the spectrums didn't change much. When the refractive index was 1.795 , we can see the grating-assisted radiation coupling took place in the longer wavelength region $(>1540 \mathrm{~nm})$. With 1.80 index cladding, output light has almost disappeared. Note that the values of refractive index of the index matching oil are dependent on the wavelength. The provided values by the manufacturer were measured at visible wavelength. The measured coupling 
efficiency of the grating was very low, which is believed due to the inaccuracy of dicing saw (normally nanometer order accuracy is required) and surface roughness.

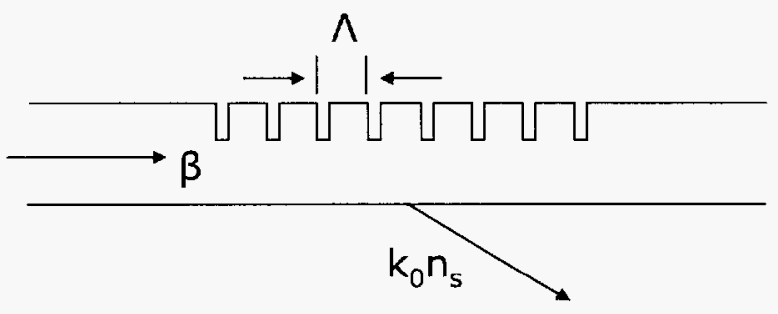

(a)

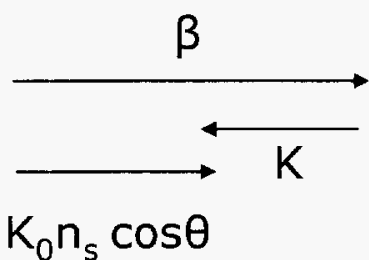

(b)

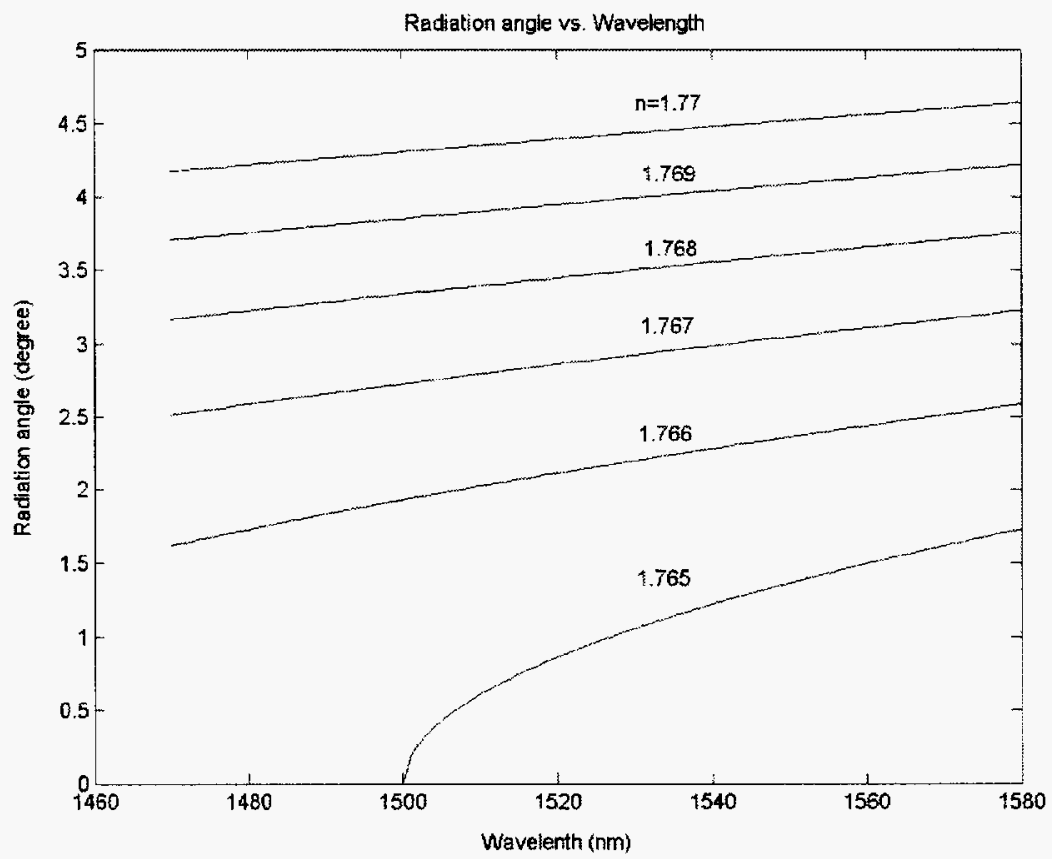

(c)

Fig. 1.4 Wavelength dependence of radiation mode coupling

\subsubsection{Demonstrate the possibility of using super continuum highly broad band source}

In general, the number of grating-based multiplexed fiber sensors is limited by the bandwidth of the source. The bandwidth of normal tunable source is around $100 \mathrm{~nm}$. To increase the number of multiplexed sensors, we need wider bandwidth for the light source. 
A supercontinuum light could be a solution for large scale sensor systems. Supercontinuum generation can be achieved in a photonic crystal fiber. Since the core radius of a certain photonic crystal fiber can be only $1 \sim 2$ micron order, nonlinear effects to cause the supercontinuum are greatly enhanced and the white light generation process easily takes place with much less input light intensity. Figure 1.6 shows the experimental setup for supercontinuum generation and generated white light source. The measured spectrum was across the entire bandwidth of OSA $(600 \sim 1700 \mathrm{~nm})$. As the spectral fluctuation of the generated white light frequently occurred by small disturbance of input coupling, it couldn't be used as an actual light source for the sensor. Once this problem is resolved, it will be a promising candidate for large scale sensor array system.

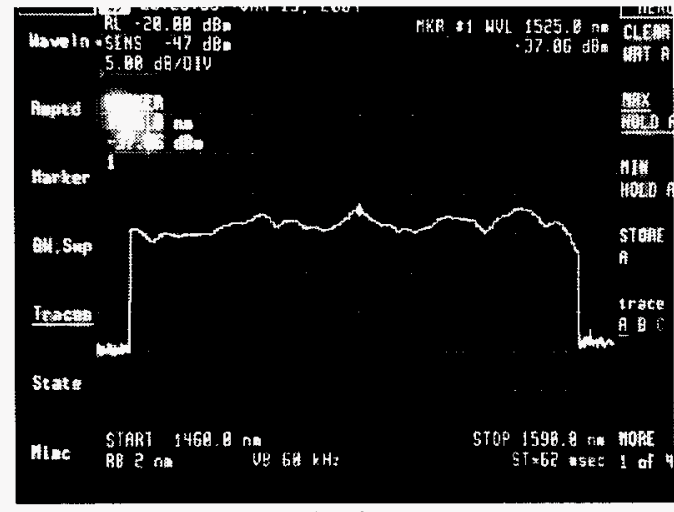

(a) air

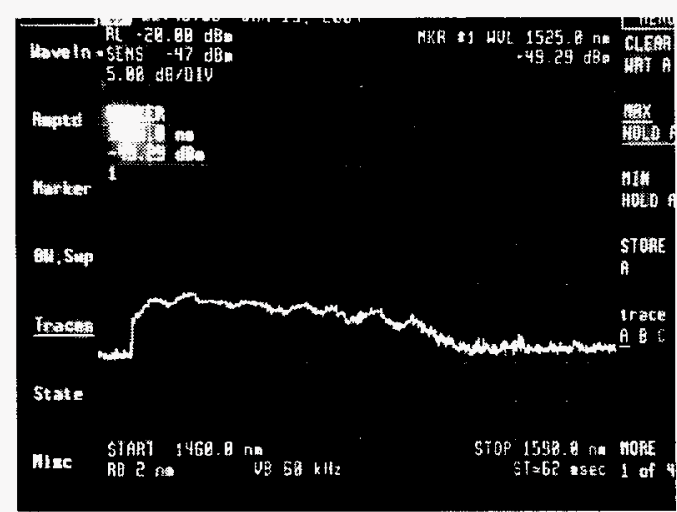

(c) 1.795

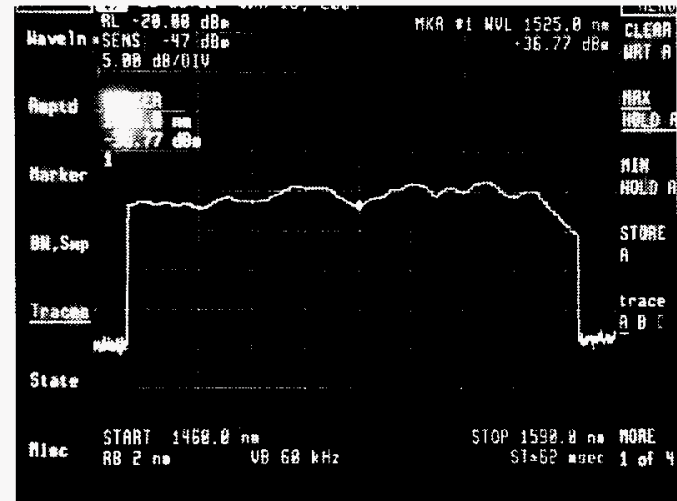

(b) 1.79

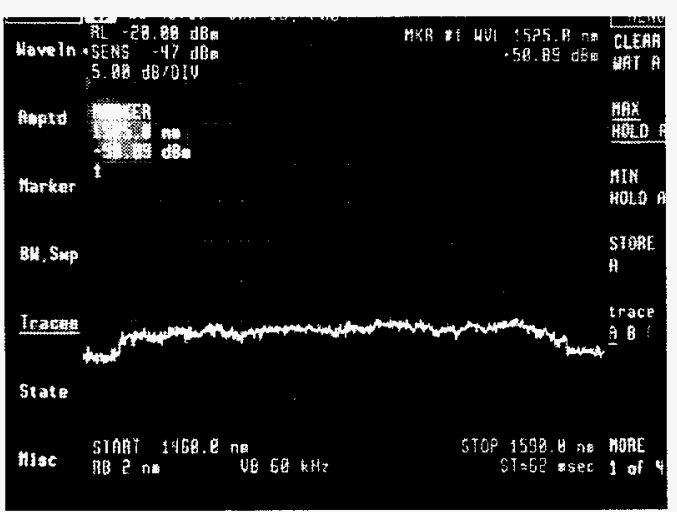

(d) 1.80

Fig. 1.5 The output spectrum of the sapphire fiber with surface gratings for different cladding refractive indices. 

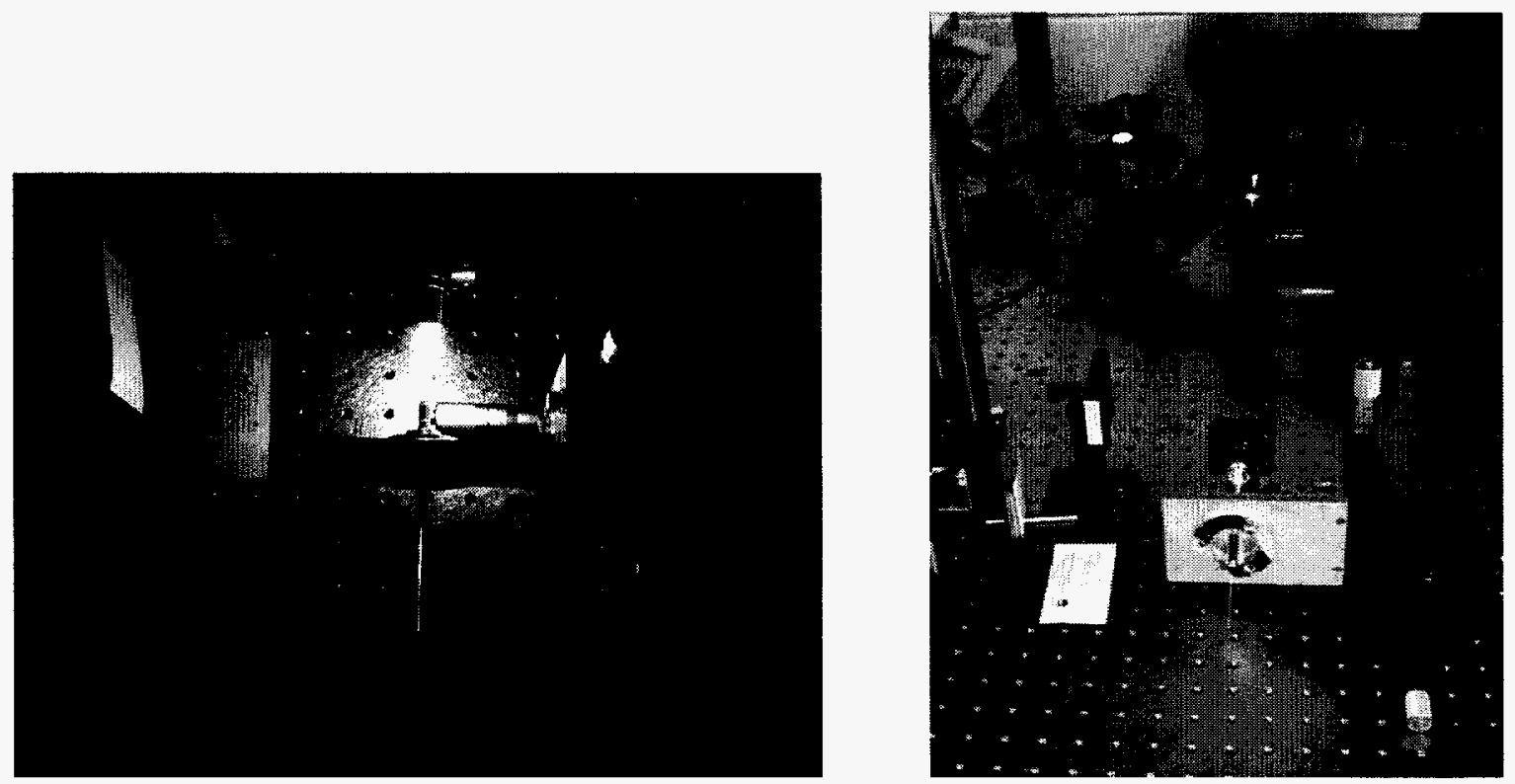

Fig. 1.6 Supercontinuum white light source generation for fiber sensor systems.

\subsubsection{Investigate the more accurate patterning and micro-structuring method than} mechanical dicing

So far, the problems we've encountered are

- Poor spatial resolution of mechanical dicing method

- Shallow grating depth of chemical etching method

- Surface roughness of structured fiber

Especially, if want to make fiber Bragg grating (FBG) in the sapphire fibers, we need submicron patterning $(\sim 200 \mathrm{~nm})$ capability. To resolve those problems, we investigated a new fabrication method using the following fabrication steps, which is illustrated in Fig. 1.7 .

- Fine polishing

- Lithography or e-beam lithography 
- Mask patterning

- High density plasma etching (ICP-RIE)

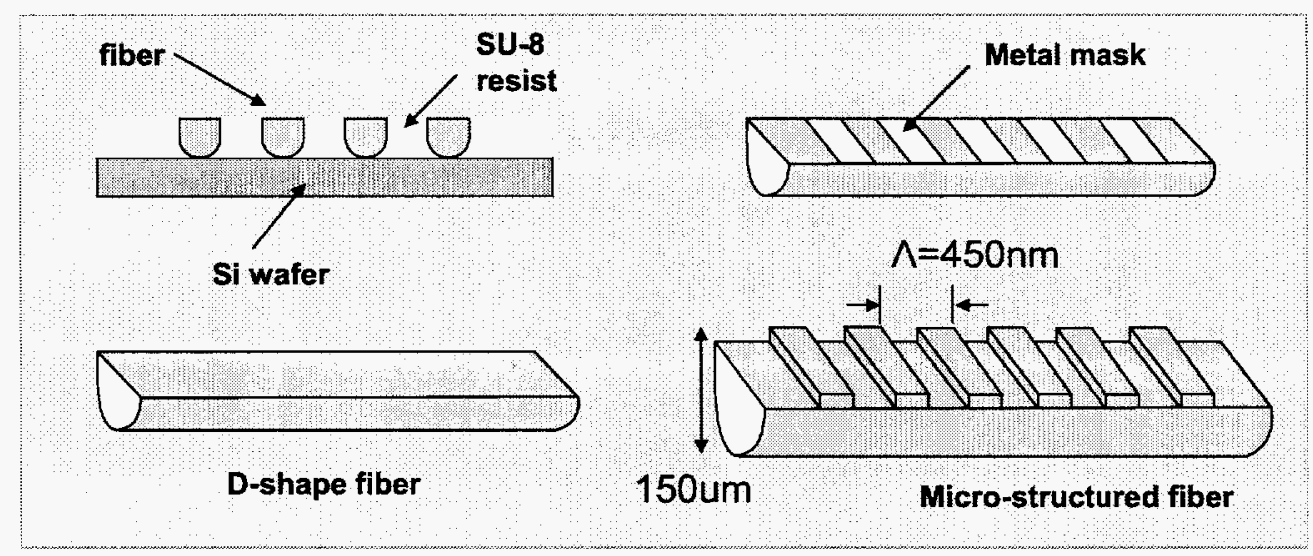

Fig. 1.7 An illustration of a fabrication process for sapphire fiber FBG grating by plasma etching.

For the accurate patterning, the precise lithography process is required, which means well polished flat surface is needed for spin coating uniform photoresist layer over the sample. It can be accomplished by putting the fibers on the silicon wafer, filling the gap with thick negative photoresist SU-8, post-baking the resist and then polishing the surface. The fibers will have D-shape as the result of polishing step. Once the surface is made flat, we can use the standard micro-fabrication methods. Using normal lithography or e-beam lithography method, the accurate pattern can be defined in the photoresist layer. For transferring the pattern into the sapphire fiber, high density plasma etching (ICP-RIE) process can be used. With $\mathrm{BCl}_{3}: \mathrm{Cl}_{2}$ chemistry and $800 \mathrm{~W}$ RF power, the etch rate of sapphire can reach $350 \mathrm{~nm} / \mathrm{min}$.

\subsubsection{Successfully fabricating in-fiber gratings in harsh environment optical fibers by high power laser system}

Typically, fiber gratings are generated by exposing the ultraviolet (UV)-photosensitive core of a germanium-doped silica core optical fiber to a spatially modulated UV laser beam in order to create permanent refractive index changes in the fiber core. A limitation of the UV induced fiber Bragg gratings, especially for high temperature sensor applications, is that operation of the sensor at elevated temperatures results in the erasure of the UV induced index modulation of the grating. Also, this grating-based method can not be directly applied to sapphire fibers due to lack of photosensitivity of sapphire fibers. Micro-machining method might be a logical solution for gratings in sapphire, but it has encountered the problems of poor spatial resolution, shallow grating depth and surface roughness. 
In recent years, the difficulty of long-term thermal stability of FBGs in a harsh environment has been largely alleviated by the development of new FBGs inscribed by infrared (IR) femtosecond lasers. Growing interest in femtosecond laser inscription is based on the fact that the physical mechanism of this process is essentially different to that of UV inscription. As a result, the physical properties of the femto-inscribed structures and devices are different from those of the UV-produced equivalents. The refractive index change in femtosecond-inscribed gratings is believed to be initiated by formation of localized plasma in the bulk of the material causing densification of the lattice. The process involves highly nonlinear photo-ionization, thus requiring high intensity of light and it only occurs in a tightly focused laser beam. Fmeto-inscription does not depend strongly on formation of defects. As a result, the decay of defects caused by thermal annealing is likely to be insignificant in the femto-inscribed structures. Hence, femtosecond inscription is able to produce structures with much improved thermal robustness compared to UV-written structures. And these mechanisms are universal and allow us to perform three-dimensional refractive- index patterning and to fabricate complicated photonic structures in practically all transparent materials including silica fiber and sapphire single crystal fiber.

We have optimized our grating written system; the fabrication system is robust and fast, less than five minutes for each grating. Our fiber grating writing scheme is based on a near field phase mask method. Laser pulses of $150-\mathrm{fs}$ duration that are generated by a Ti:sapphire amplifier at a wavelength of $800 \mathrm{~nm}$ with a pulse repetition rate of $1-\mathrm{kHz}$. The pulse energy is around $1 \mathrm{~mJ} /$ pulse, which is strong enough to write Type II IR gratings. 


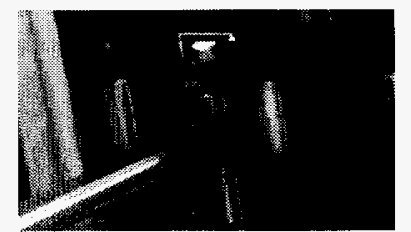

The Ti:sapphire femtosecond laser system includes the seed laser, femtosecond pulse regenerative and multipass Ti:Sapphire amplifier system, namely the Titan system

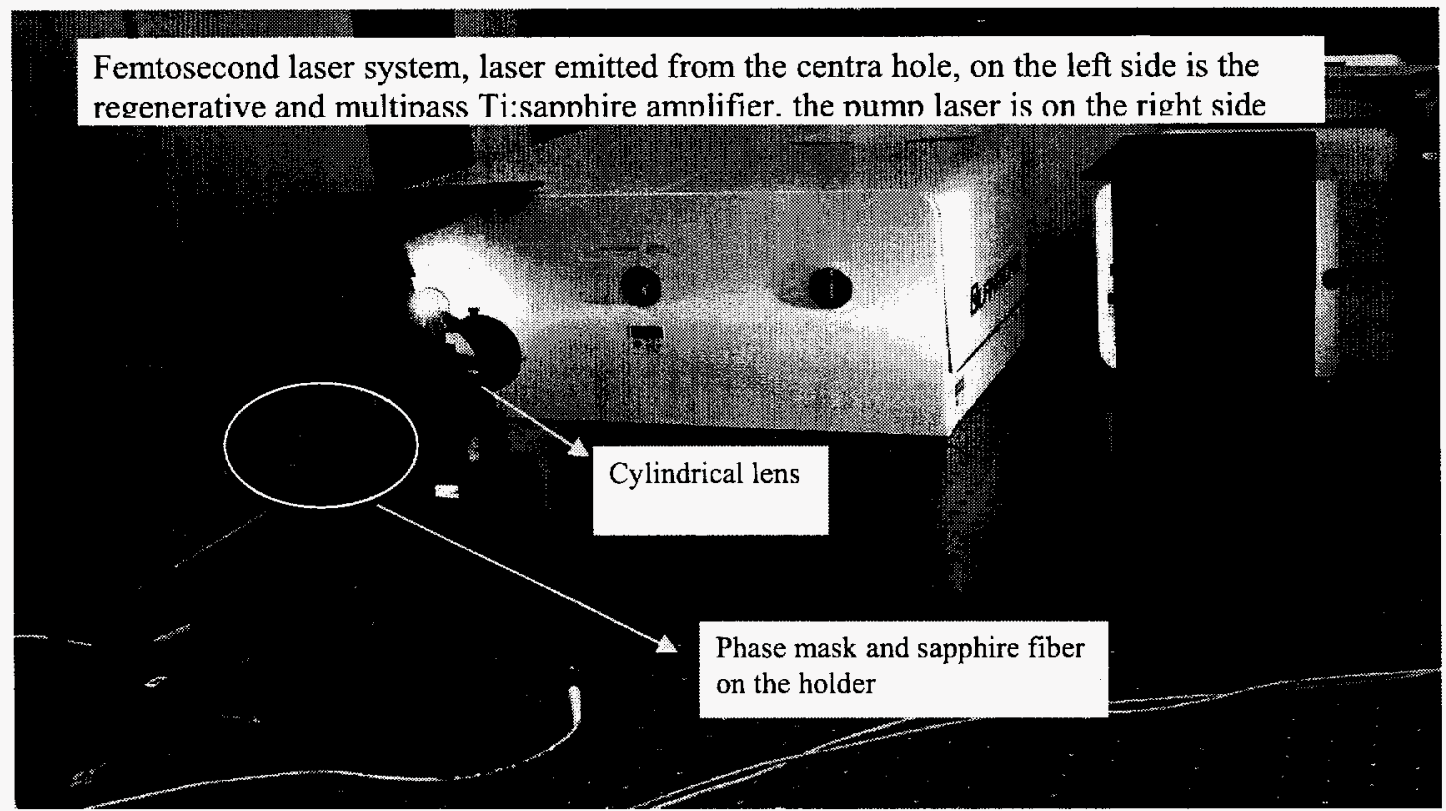

Fig. 1.8 (a). Ultrafast (femtosecond) experimental system for fabricating harsh environment fiber optic

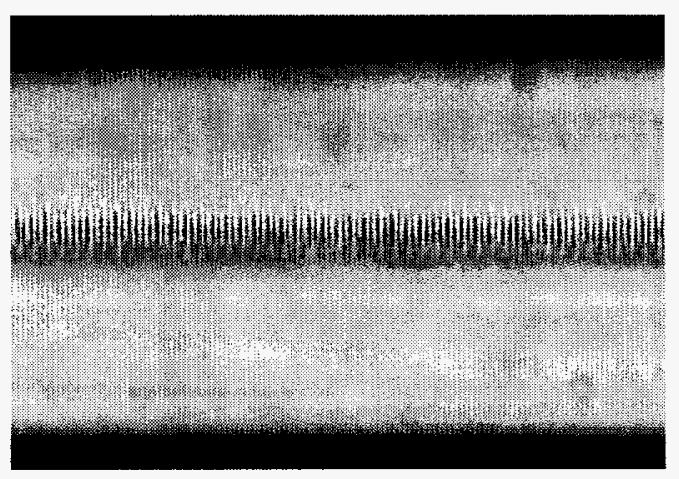

Fig. 1.8 (b). Microscopic image of Bragg grating fabricated in a single mode (SM) silica fiber.

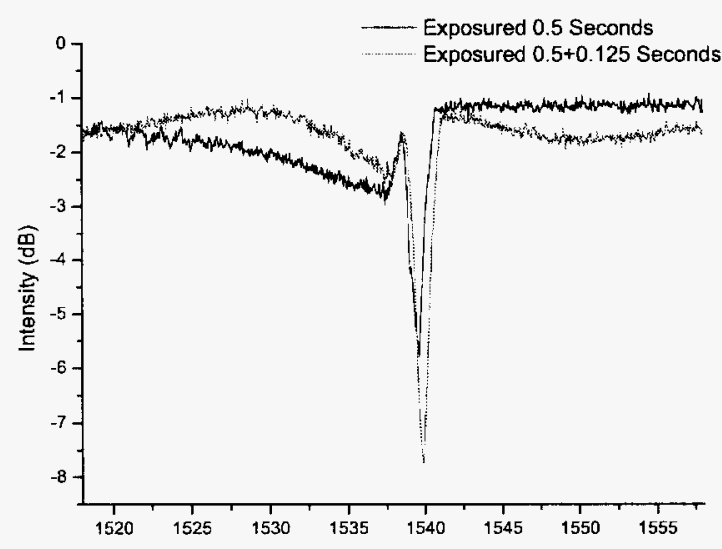

Fig. 1.8 (c). The spectrum of the grating fabricated by the ultra short laser pulses in SM fiber. 


\subsubsection{Perform temperature test with femtosecond laser inscribed FBG in high temperature furnace.}

The grating was attached to high temperature alumina ceramic rod with Hi-purity alumina ceramic paste.
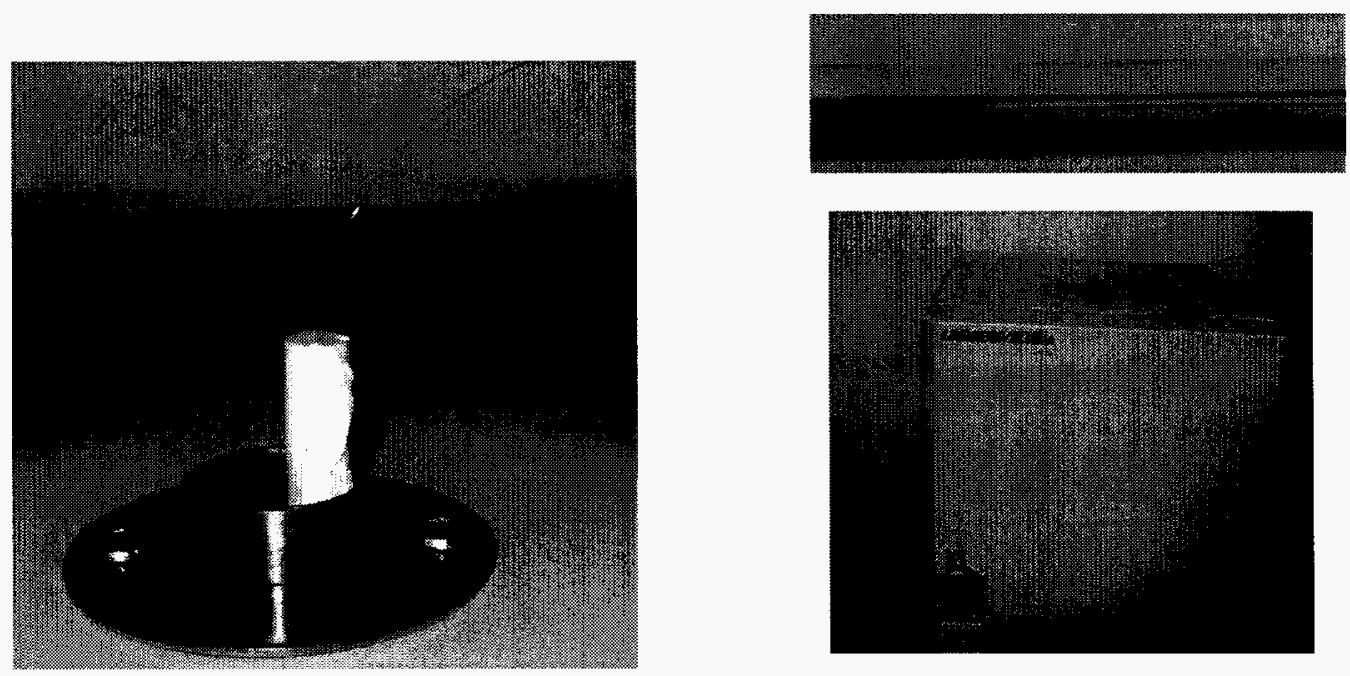

Fig. 1.9. Experimental set-up of high temperature testing

The fiber with grating was buttcoupled to a four-port coupler made of single mode silica core fiber. The signal from the tunable laser was launched through a single-mode fiber into the input port of the coupler and the optical spectrum analyzer (OSA) at the output port collected the reflected signal from the grating. The end of the fiber that contained the Bragg grating along with the supporting rod was then put into the furnace and the reflection signal was measured. From the experiment results shown in the following figures, we can see silica FBG fabricated by femtosecond laser inscription has good linear response for high temperature measurement. 


\section{Resonant wavelength shift with temperature}

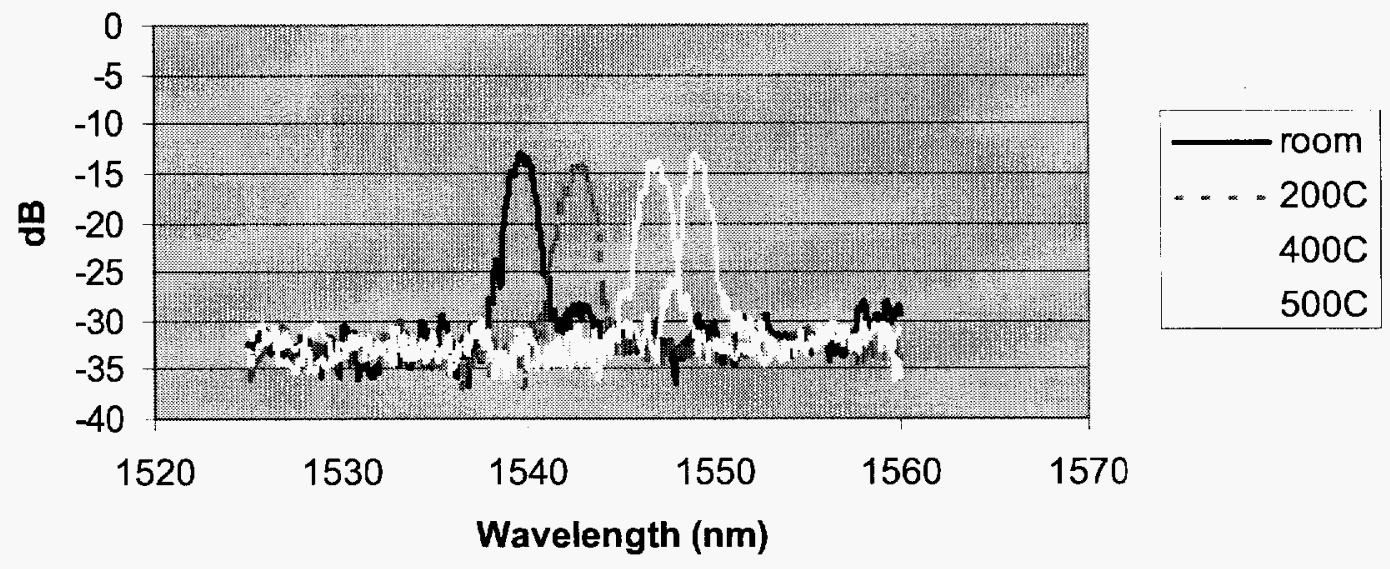

Fig. 1.10 (a). Measured grating spectrum with different temperature

\section{Resonant wavelength shift with temperature}

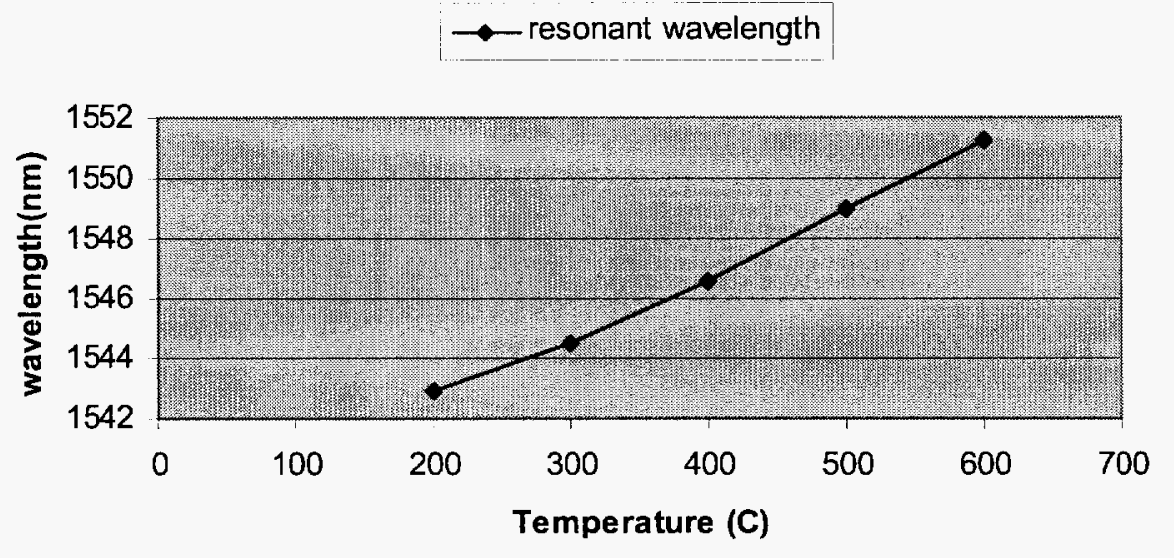

Fig. 1.10 (b) Grating resonant wavelength shifted as a function of temperature

1.2.6 Demonstrate stable performance of our high temperature sensor in Mckenna Burner. 


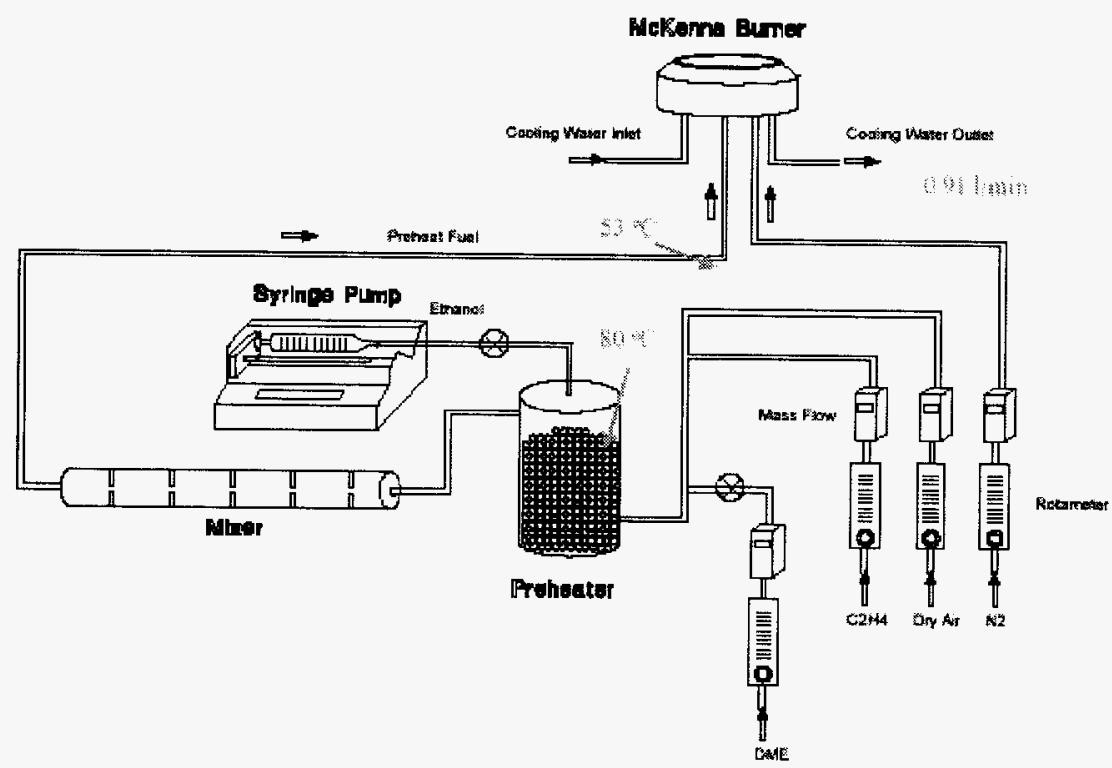

Fig. 1.11 (a) Schematic diagram of McKenna burner system

In the above figure shows the burner system which includes the fuel, additive, and air supply. An unconfined flat laminar premixed flame was established using a McKenna Burner, which consists of a 60.2-mm-diameter water-cooled porous-plate.

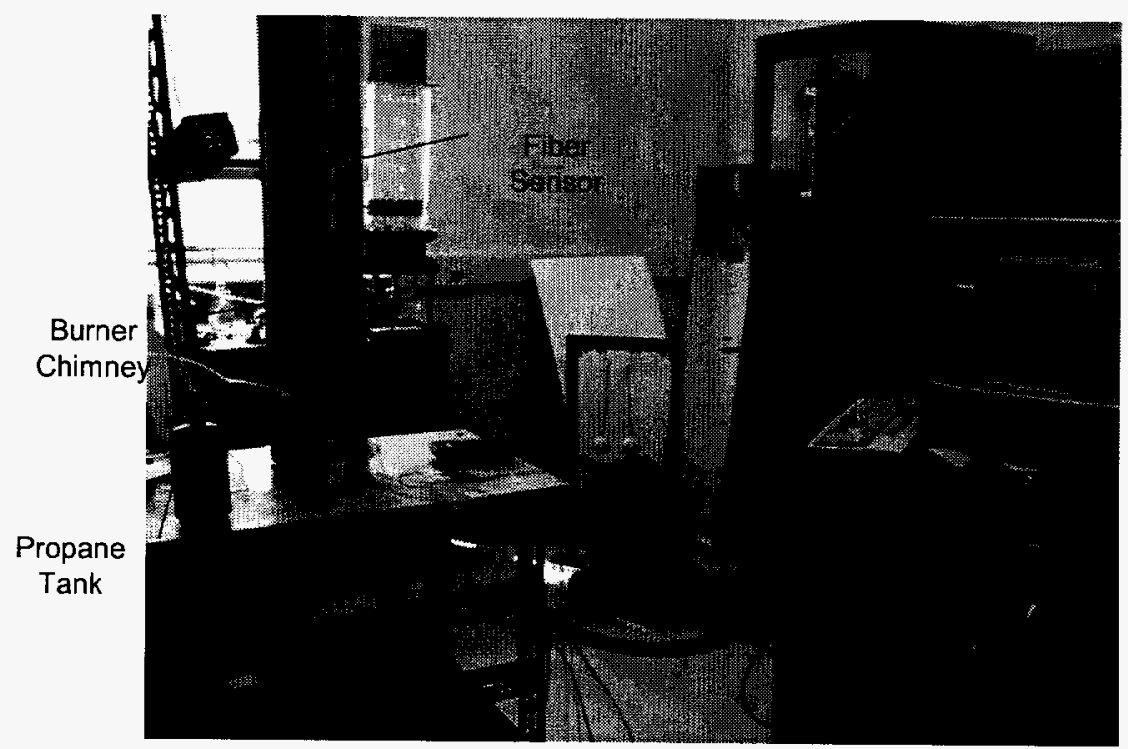

Fig. 1.11 (b) Real world experiment set-up of McKenna burner system 


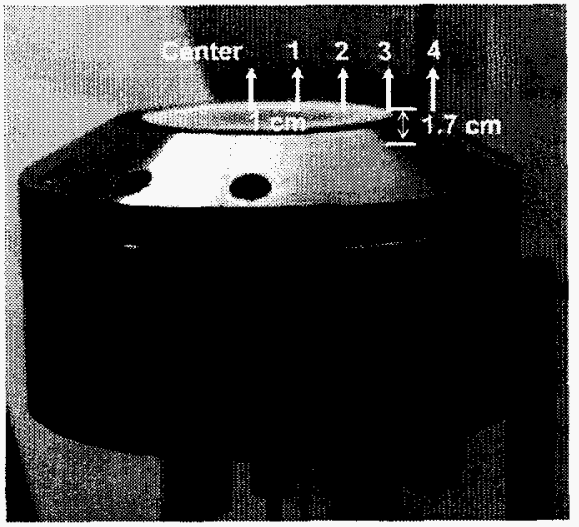

Fig. 1.12 (a) Horizontal direction along burner.

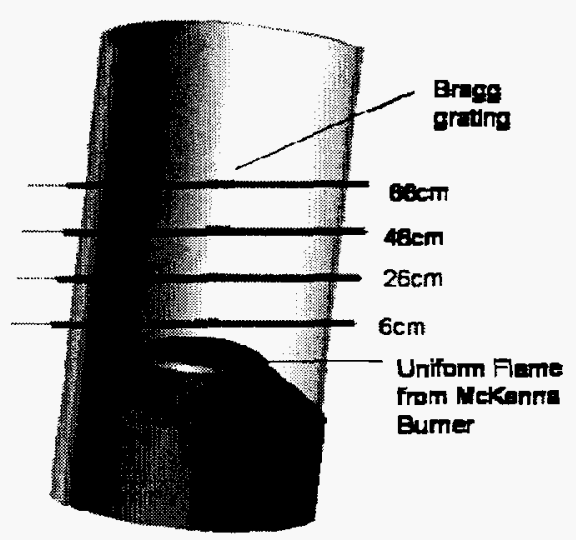

Fig. 1.12 (b) Vertical directions along burner.

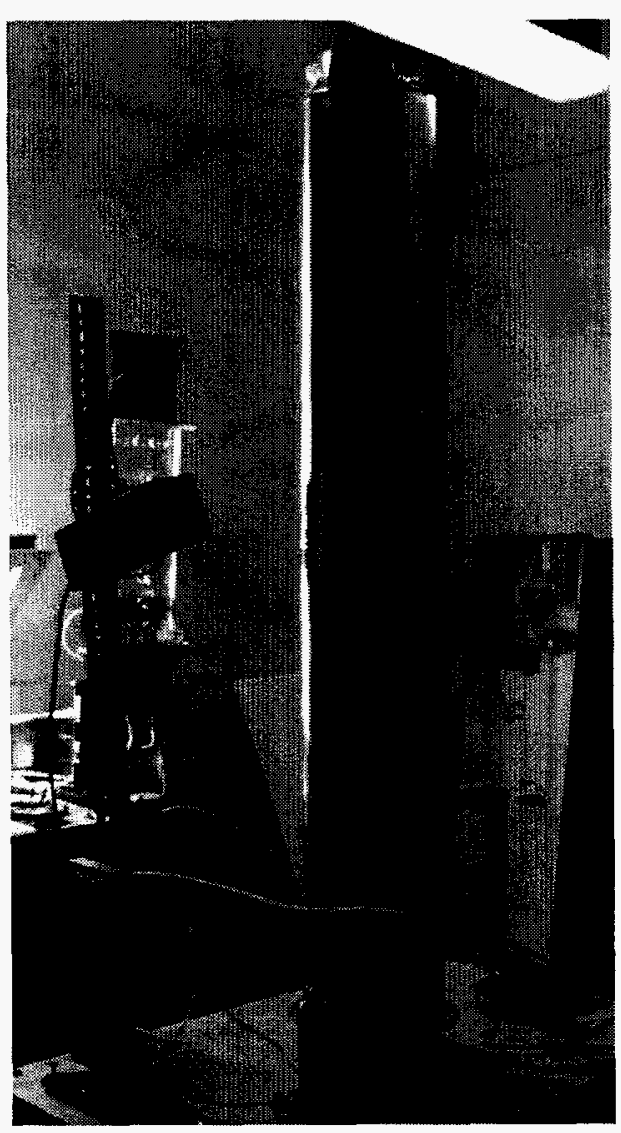

Fig. 1.12 (c) Holes distributions in the chimney along vertical direction.

The burner is covered by chimney with holes in it, and the ceramic rod with fiber grating attached is inserted in the holes to measure the temperature profile of the burner. In horizontal direction, measurement is taken at $1.7 \mathrm{~cm}$ above the burner surface plate. And 5 positions are chosen. The distance between each position is $1 \mathrm{~cm}$. In vertical direction, the measurement is conducted at four different heights above the burner surface. 


\begin{tabular}{|c|c|c|c|}
\hline \multicolumn{2}{|c|}{ Vertical direction } & \multicolumn{2}{c|}{ Horizontal direction } \\
\hline $\begin{array}{c}\text { Height } \\
\text { above the } \\
\text { flame }(\mathrm{cm})\end{array}$ & $\begin{array}{c}\text { Temperature } \\
\left({ }^{\circ} \mathrm{C}\right)\end{array}$ & Position & $\begin{array}{c}\text { Temperature } \\
\left({ }^{\circ} \mathrm{C}\right)\end{array}$ \\
\cline { 3 - 4 } & Center & 980 \\
\hline 6 & 730 & 1 & 940 \\
\hline 26 & 180 & 2 & 900 \\
\hline 46 & 138 & 3 & 690 \\
\hline 66 & 127 & 4 & 400 \\
\hline
\end{tabular}

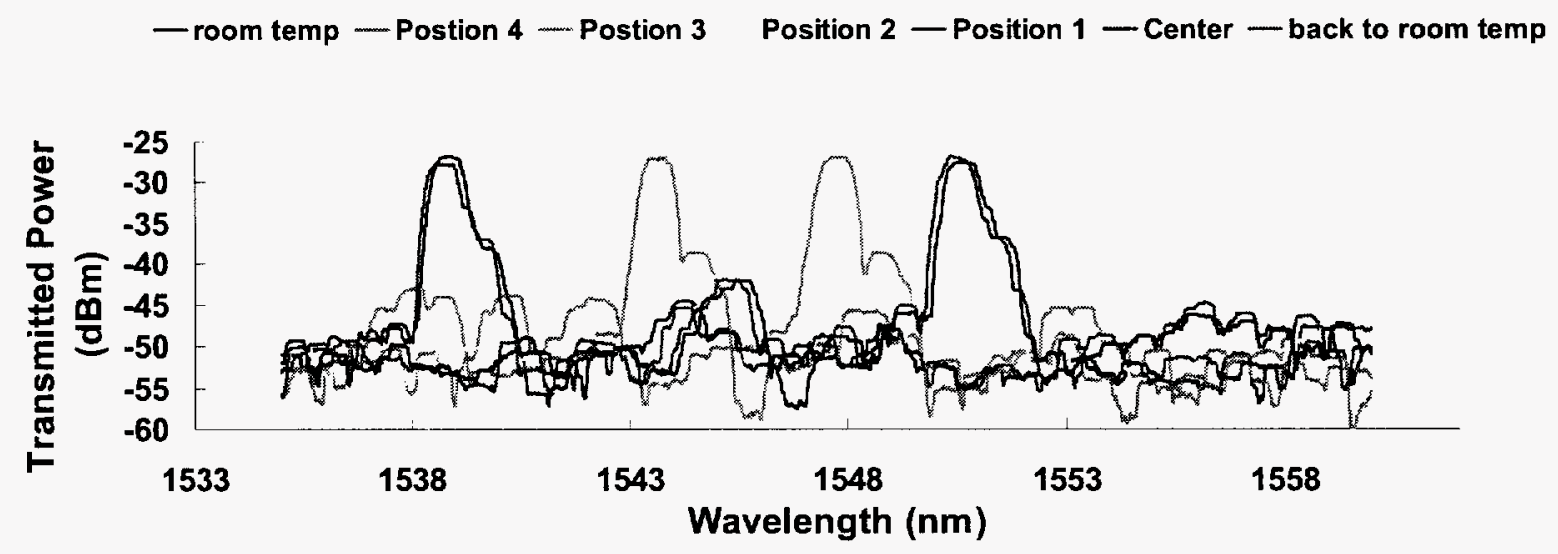

Fig. 1.13 (a) Grating spectrum measured with different locations along the vertical direction.

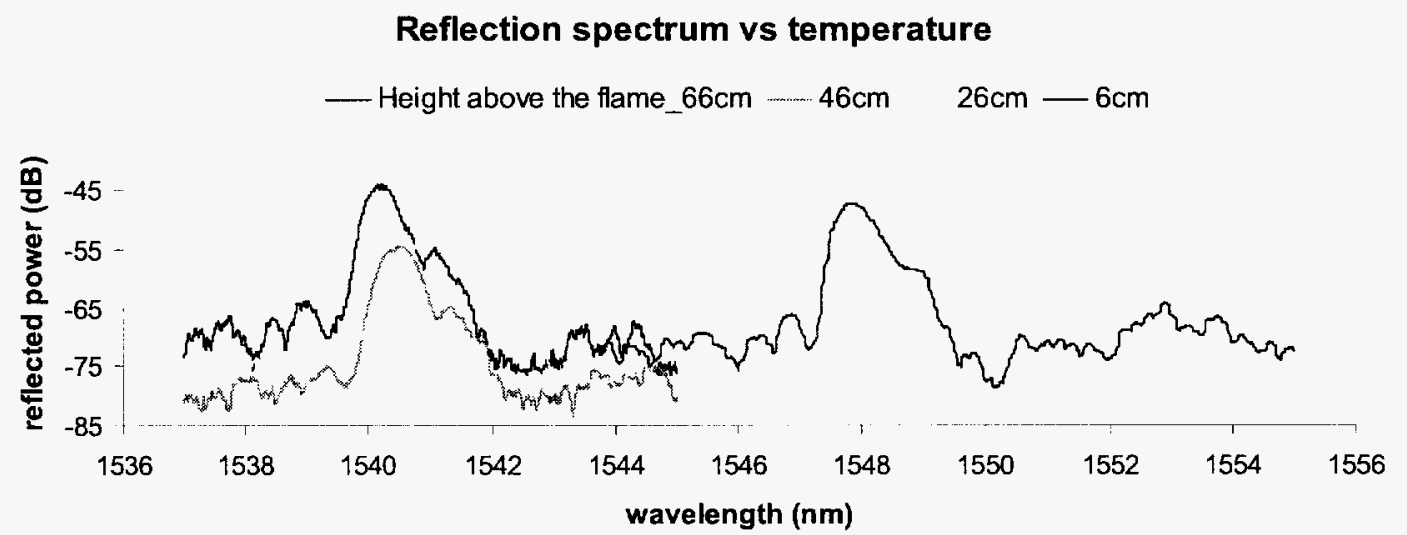

Fig. 1.13 (b) Grating spectrum measured with different locations along the horizontal direction. 
Experiment shows the temperature sensitivity is around $0.013 \mathrm{~nm} /{ }^{\circ} \mathrm{C}$. The temperature sensitivity at the $1540 \mathrm{~nm}$ Bragg grating wavelength is estimated to be $0.014 \mathrm{~nm} /{ }^{\circ} \mathrm{C}$. The Bragg grating in the silica single mode fiber considered in this experiment is well consistent with the theoretical predication.

\subsubsection{Demonstrate good long term stability of the femtosecond laser inscribed FBG} sensor

In order to test the long term thermal stability, the femtosecond laser induced Bragg grating was inserted in a stainless tube and put in a high temperature furnace for about ten days. The grating spectrum was monitored by a micron optics sensing interrogator.

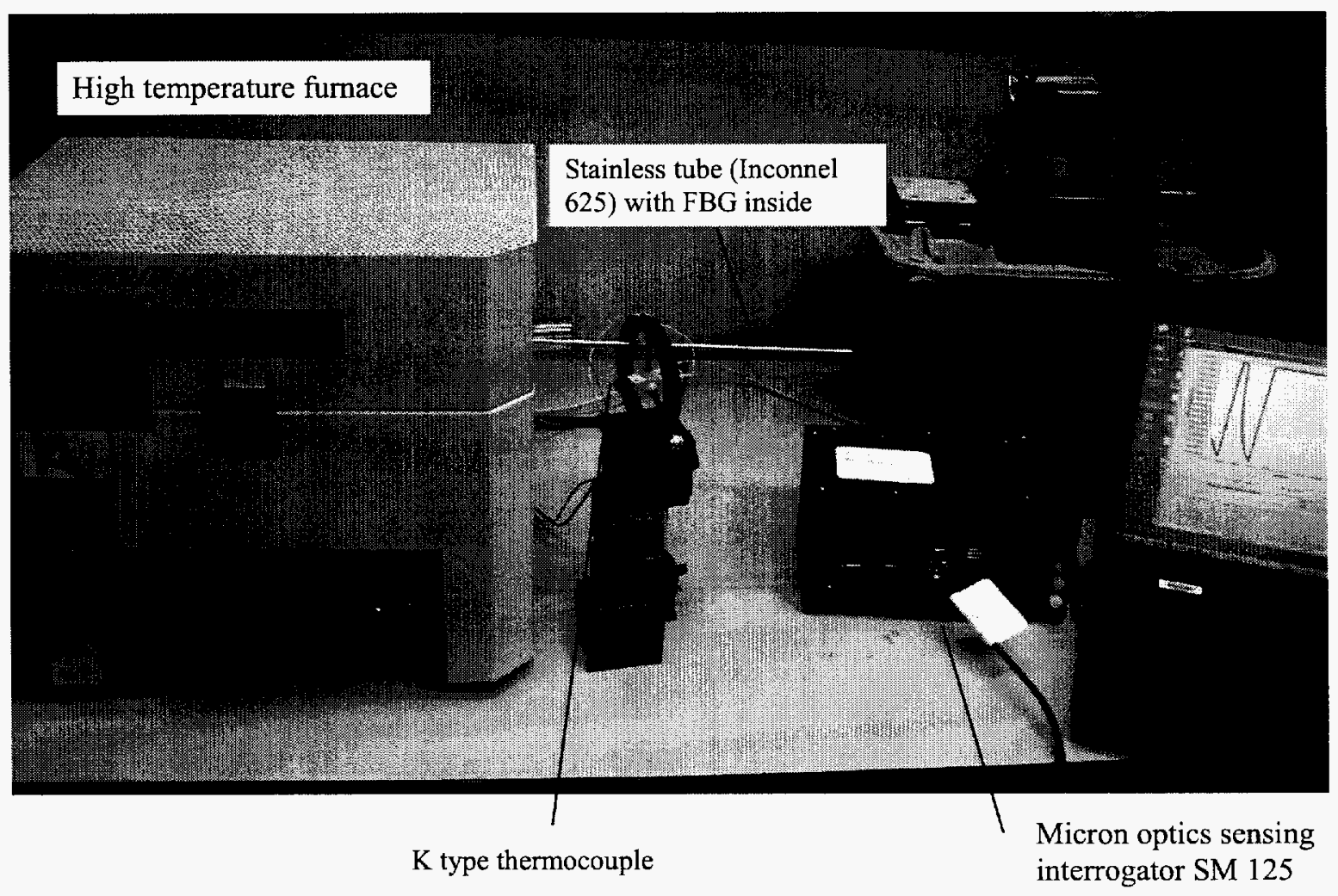

Fig. 1.14. Experimental set-up of durability test 

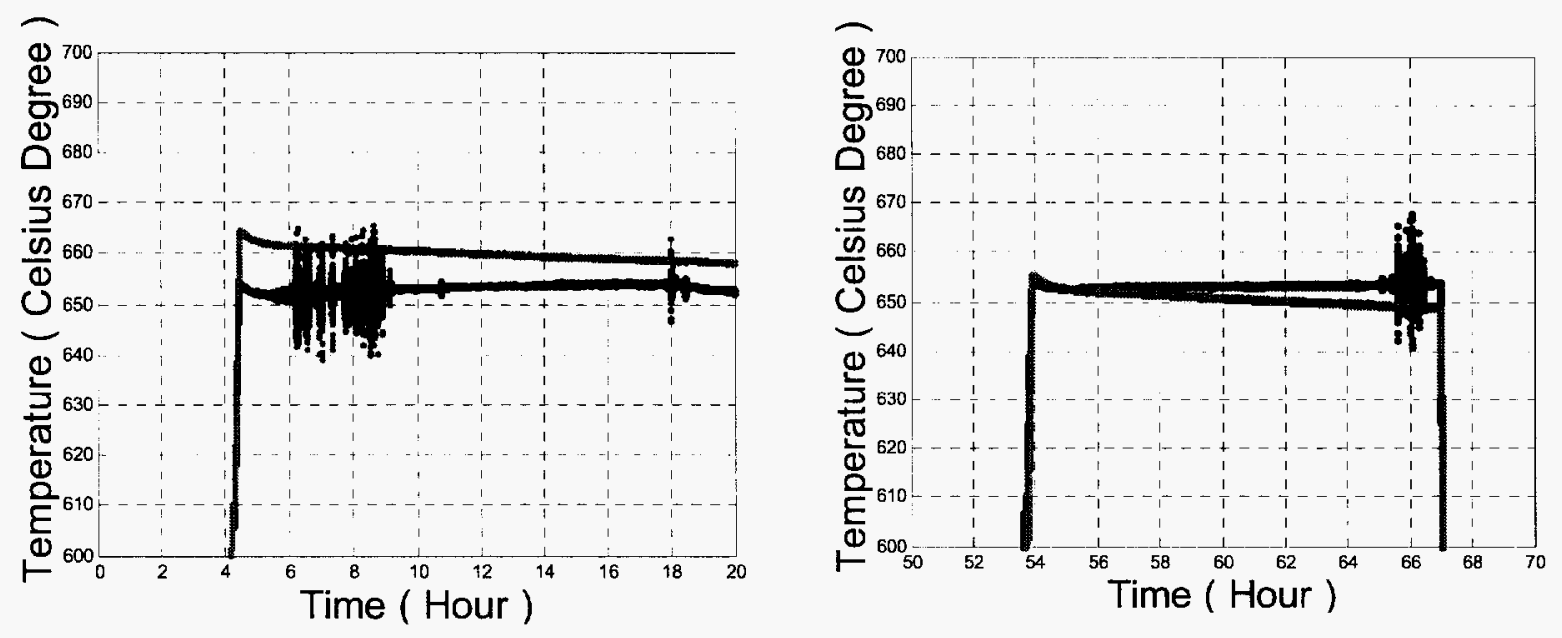

Fig. 1.15. A comparion between FBG measurement results and thermocouple results

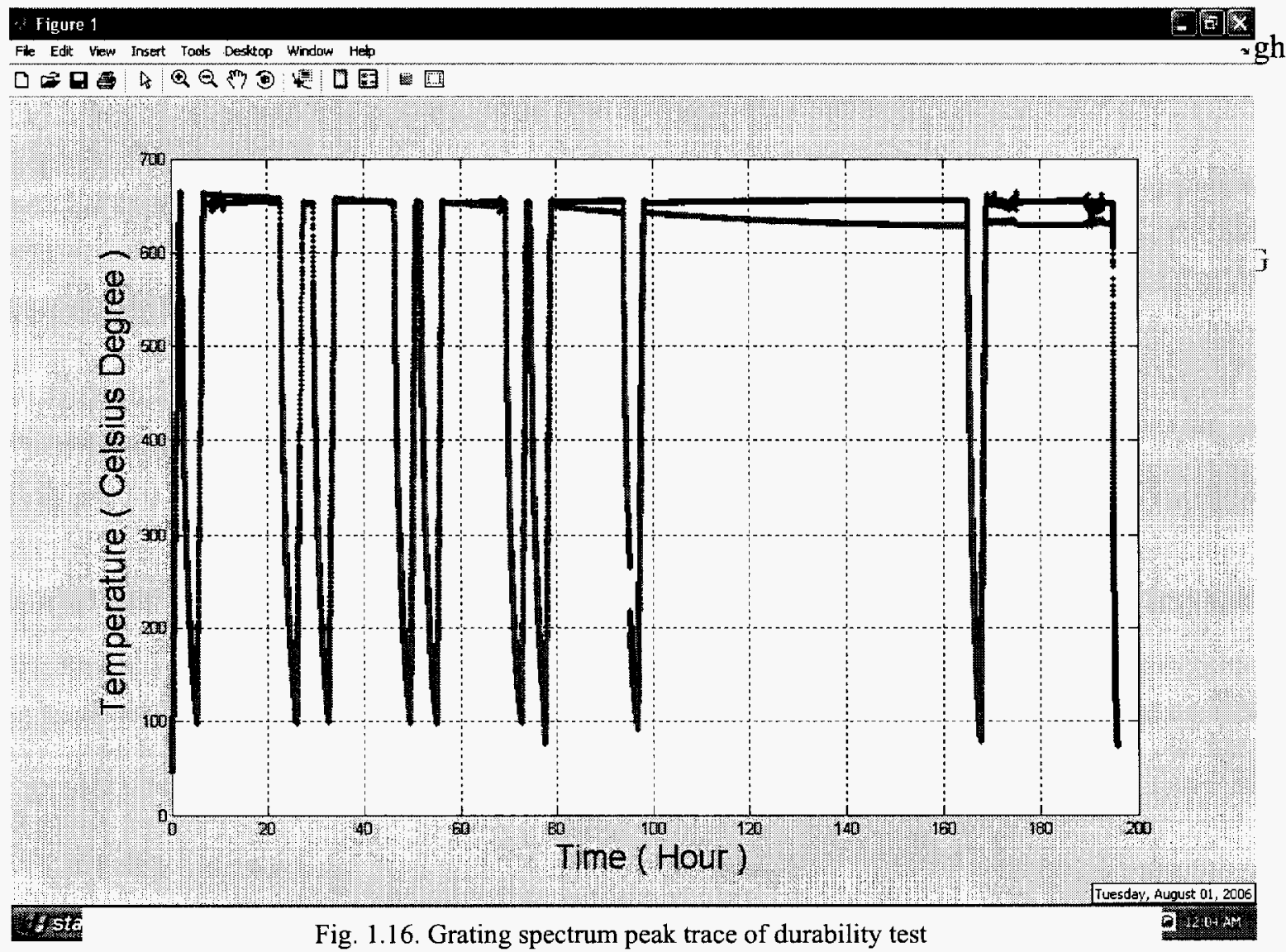


Experimental results show that FBG drift at fixed temperature after heated for about 80 hours, and it has a maximum value, when reached the maximum, the spectrum is stable again afterwards. Since the Resonant peak shifted to shorter wavelength during the drifting, according to Bragg condition, the possible reason will be the decreasing of Neff (effective refractive index of fiber core) or $A$ (grating period). And grating structure degradation may cause $\Lambda$ decreasing, but for a typical silica fiber $d L /(L d T)=5 \times 10-7 \mathrm{~K}-1$ and $d n / d T=10-5 \mathrm{~K}-1$, so the dominate factor should be the change of effective refractive index. Diffusion of $\mathrm{Ge}$ from core to cladding and silica crystallization may cause the change of Neff. The diffusion coefficient at different temperatures is found to be well predicted by

$$
D=D_{0} e^{-\frac{E_{4}}{R T}}
$$

At higher temperature, the diffusion coefficient is larger, this explains the fast drifting at higher temperature than lower temperature. After some time, when the diffusion reaches equilibrium, the drifting stopped. Thermocouple shows more random noise than FBG, while Regular SM Ge doped silica fiber grating shows drifting $\left( \pm 7^{\circ} \mathrm{C}\right)$ at temperature higher than $600^{\circ} \mathrm{C}$. PSU is currently investigating new fiber materials trying to overcome this drifting. In fact, since the drifting is predictable, with carefully calibration, FBG can totally replace thermal couple with much lower cost and more stable performance.

\subsubsection{Successfully demonstrate multi-parameter sensing capability}

In the harsh environment, in general, high temperature, strains and vibrations are coexisted, and it is always a big challenge to decouple these effects. Unfortunately, SM FBG has only one resonant dip in the transmission spectrum, which makes it difficult to realize multi-parameter sensing in a harsh environment.

We demonstrated a multiple parameter integrated fiber sensor that can detect vector bending and ambient temperature simultaneously with a single asymmetric multimode fiber Bragg grating. Multimode Bragg gratings were fabricated in an all-silica core fiber by an infrared femtosecond laser, which showed multiple transmission dips in the transmission spectrum. Bending and ambient temperature fluctuations affect the shapes of multiple transmission dips in different ways. In bending, different dips have different sensitivities. On the other hand, temperature fluctuations tended to influence the dips uniformly across different dips. By analyzing the changing spectrum of dips, one can distinguish the changes induced by bending or temperature fluctuations. Furthermore, the high thermal stability of Bragg gratings inscribed by an infrared femtosecond laser can make this double parameter fiber sensor work in very harsh, high-temperature environments. In the temperature sensing experiment, the FBG, as shown in Fig.1.17, was put in a furnace. One end of the fiber was connected to a broadband white-light source and the other end of the fiber as connected to an optical spectrum analyzer. The spectrum of the FBG was measured at different temperatures. 


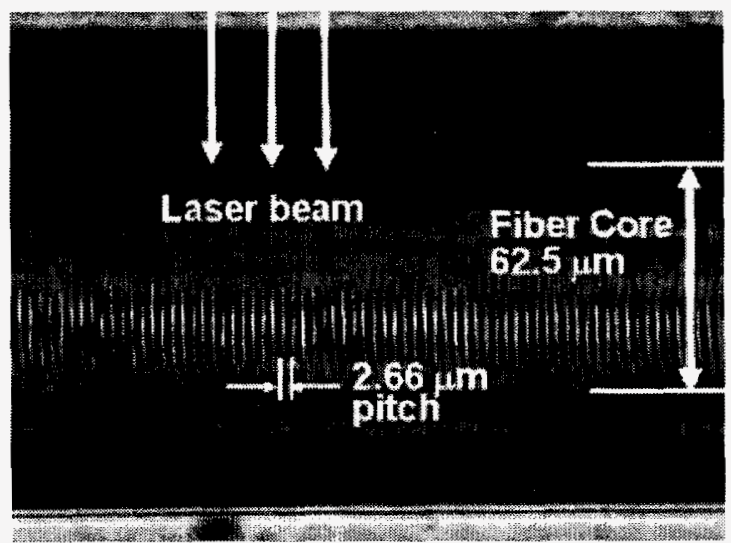

Fig. 1.17. Microscopic image of Bragg grating fabricated in a multimode silica fiber

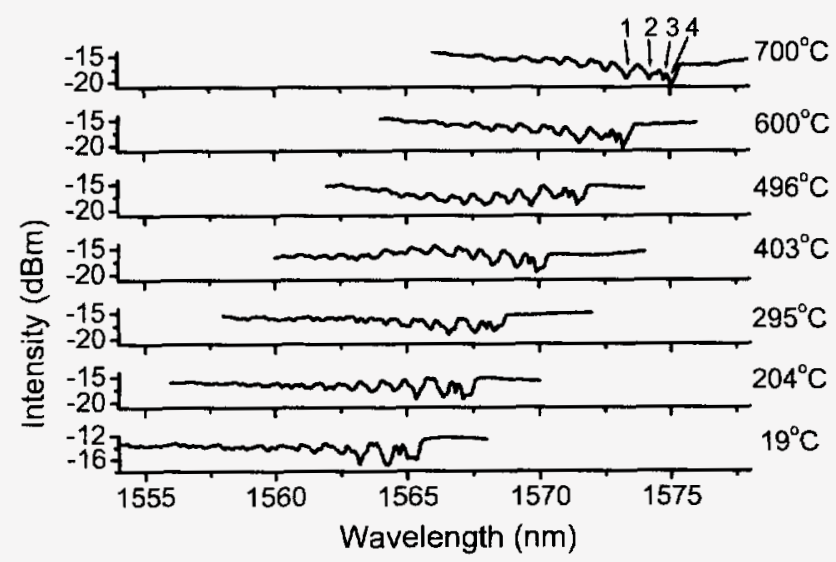

(a)

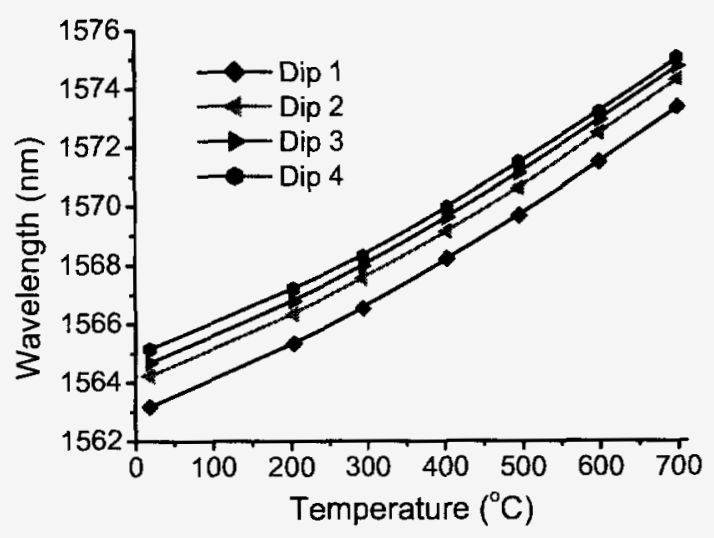

(b)

Fig. 1.18. (a) Measured spectra of a FBG in a multimode fiber inscribed by an IR femtosecond laser at a spectral range from room temperature to $700^{\circ} \mathrm{C}$. (b) Spectra of dip locations as a function of temperature for four dips.

In the bend sensing experiment the FBG was wrapped on a cylindrical surface of $25 \mathrm{~cm}$ radius. Again, one end of the fiber was connected to a broadband white light source and the other end of the fiber was connected to an optical spectrum analyzer. The spectrum was measured for the cases with and without bending. To determine the vectorial- (or directional-) sensing capability for this asymmetric FBG, the FBG was bent in two orthogonal directions and measured the spectra for both bending directions. Figures 1.18 (a) and 1.18 (b) show the measured spectra for all three cases: without bending, with bending in $x$ direction, and with bending in the $y$ direction. 


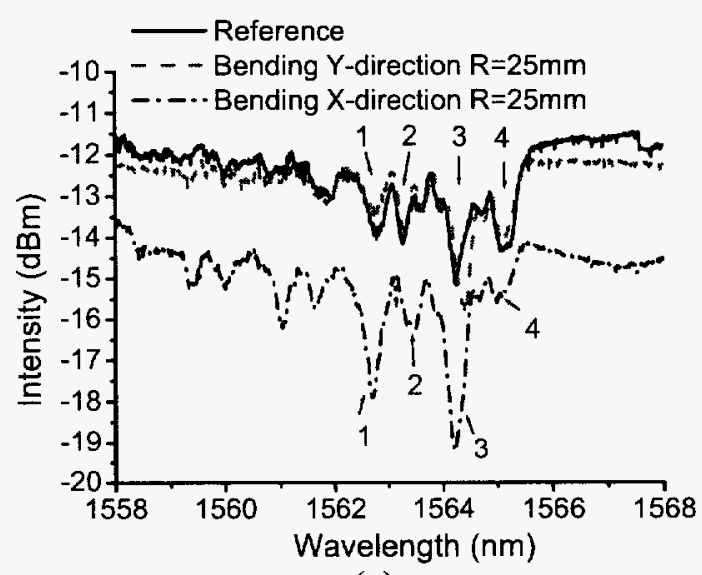

(a)

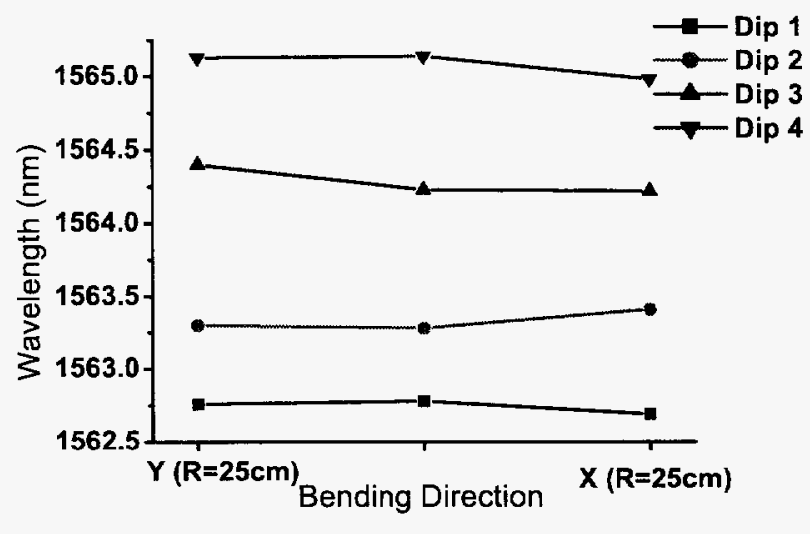

(b)

Fig. 1.19. (a) Measured spectra of FBG in multimode fiber inscribed by an IR femtosecond laser at three bending conditions: without bending (references), bending in $\mathrm{X}$ direction, and bending in the $\mathrm{Y}$ direction. (b) Spectra of dip locations as a function of bending direction for four dips.

\subsubsection{Perform high temperature sensing using sapphire fiber Bragg grating fabricated by femtosecond laser irradiation}

We have successfully fabricated gratings in both silica and single crystal sapphire fibers by the ultrafast laser system, as shown in Fig. 1.20 (a). The area with strong scattering light is the area with the grating. The diffraction pattern (upper right figure) clearly shows the grating diffraction pattern.
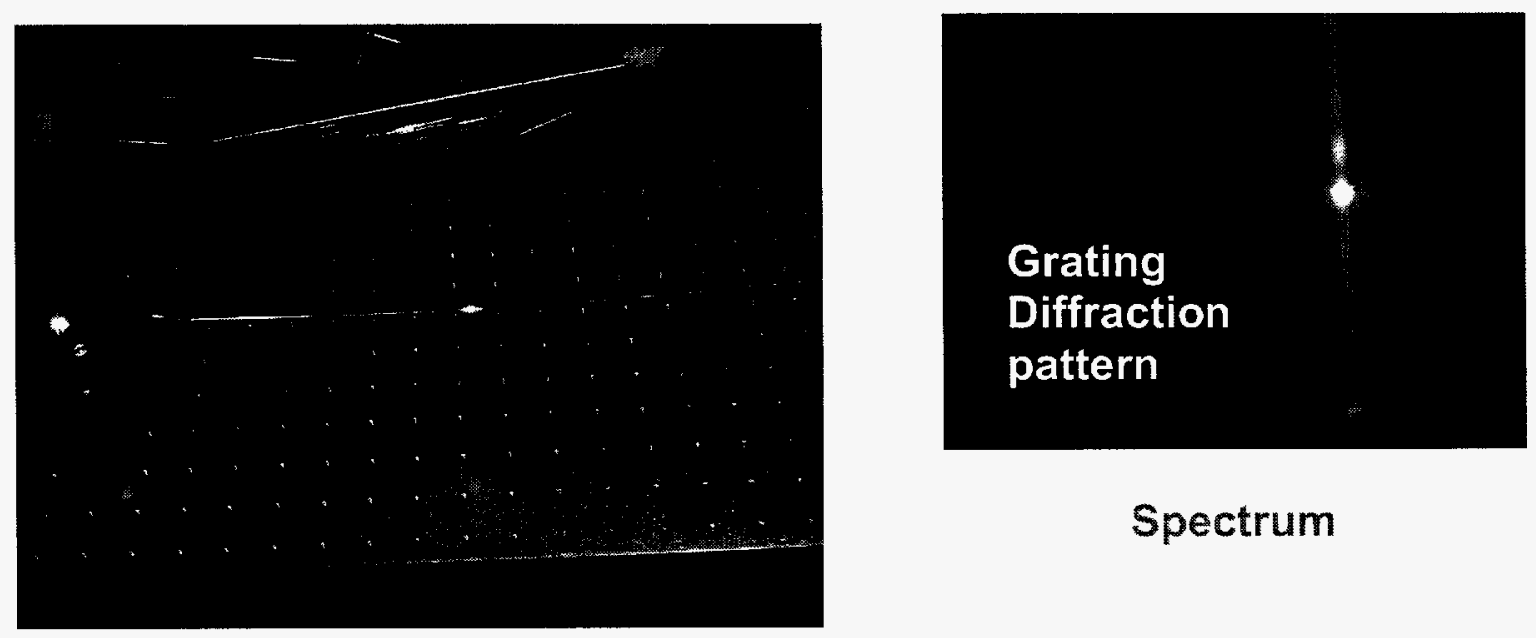

\section{Spectrum}

Fig. 1.20 (a). Fabricated gratings in single crystal sapphire fiber. 
A. High temperature sensing using sapphire fiber

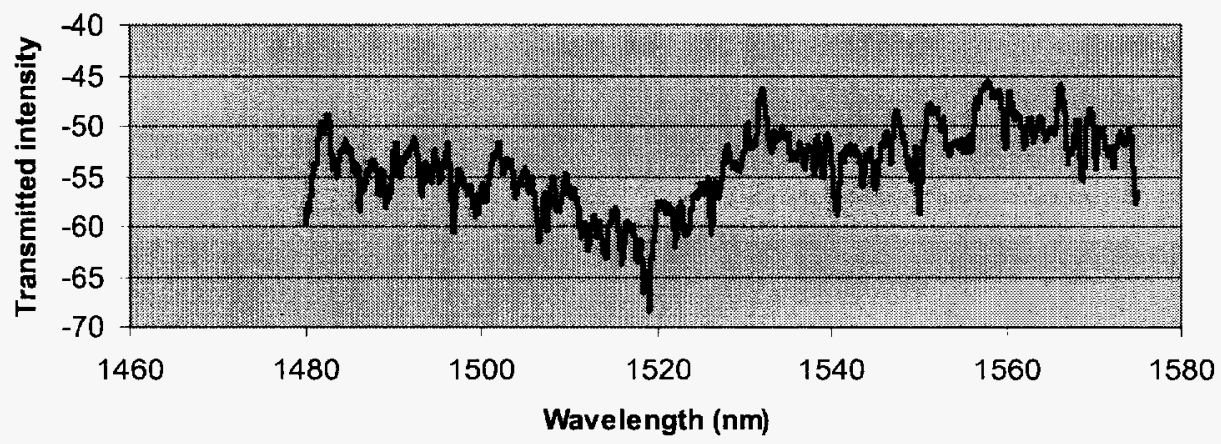

Fig. 1.20 (b) Sapphire grating transmission spectrum at room temperature

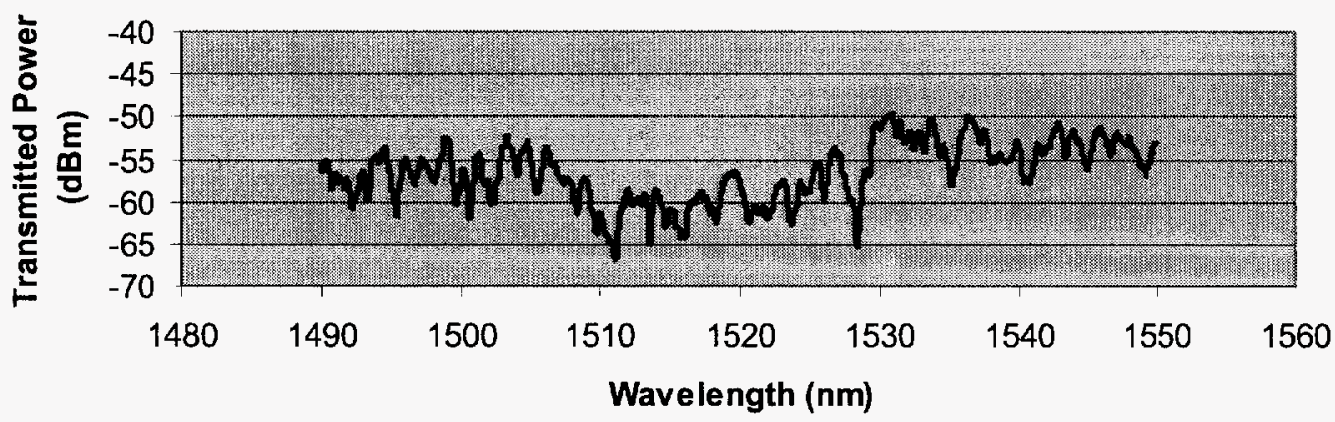

Fig. 1.20 (c) Sapphire fiber grating transmission spectrum (heated with $700 \mathrm{~F}$ solder iron)

B. mode control to suppress higher order modes.

— without mode bending - with mode bending

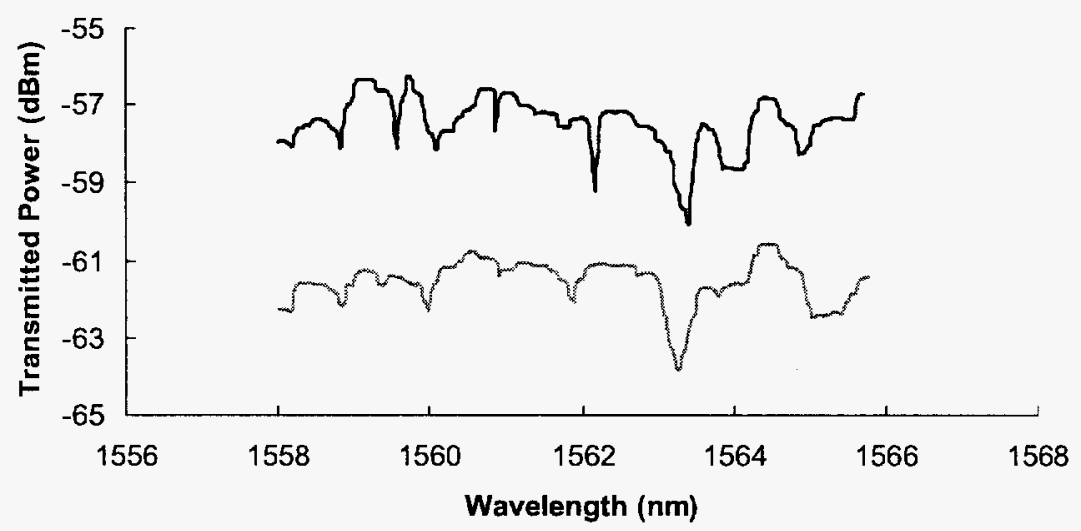

Fig. 1.20 (d) Comparison of sapphire fiber grating transmission spectrum before and after mode bending to suppress higher order modes 
We have developed advanced gratings in single crystal sapphire fibers.

- Ultra thin fiber (60 micron, regular 125 micron) (High coupling efficiency between this fiber and conventional silica fiber).

- Advanced coupling with mode control.

- Transmission spectrum was measured and grating spectrum shifted with temperature. Operation temperature can go as high as $2000^{\circ} \mathrm{C}$.

Sapphire is highly multimode, core only and sensitive to vibrations and grating depth is much shallower than silica fiber. At this moment, it may not be the best choice for our application. Regular silica fiber FBG can stand temperature as high as $1000^{\circ} \mathrm{C}$, although it has the problem of drifting, but the mount is predictable (according to diffusion equation).

\subsubsection{Develop highly sensitive long period gratings (LPG) by electric arc.}

Figures 1.21 (a) and (b) show the picture and corresponding drawing of electric arc grating fabrication system.

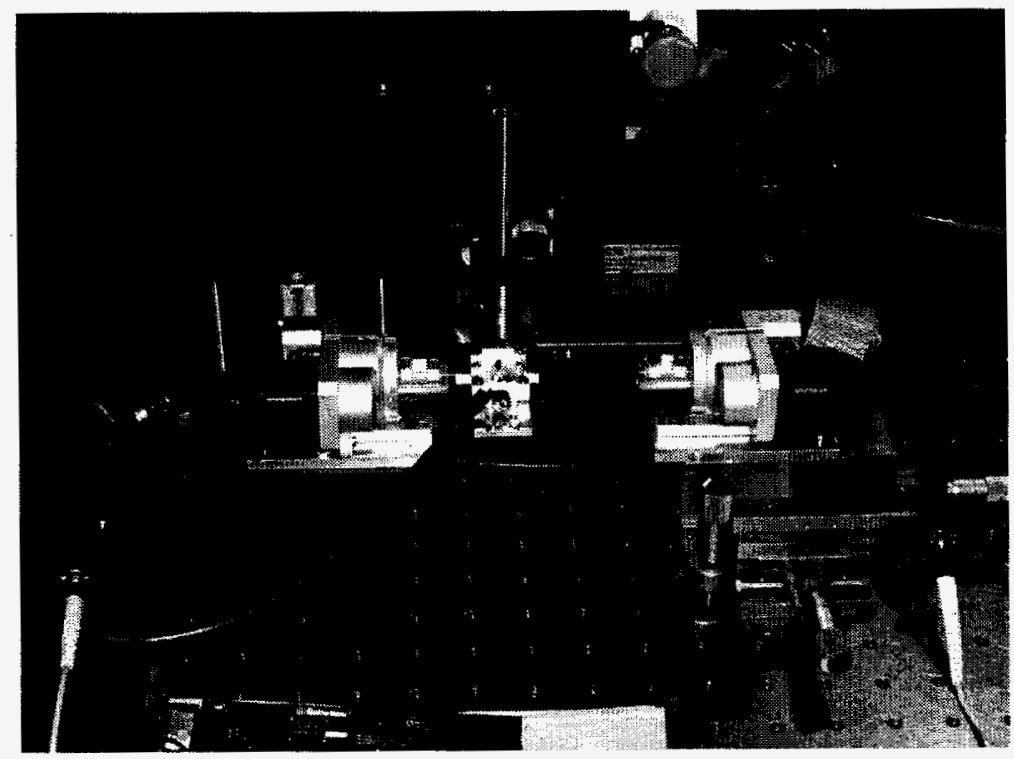

Fig. 1.21 (a) A picture of electric arc grating fabrication system. 


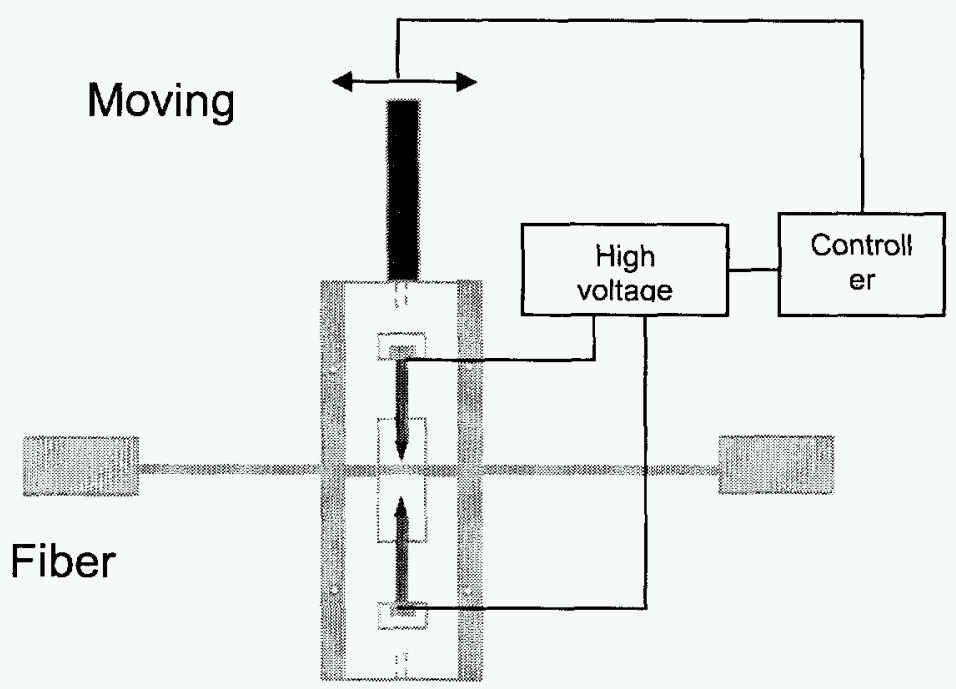

Fig. 1.21 (b). The corresponding drawing of electric arc grating fabrication system.

A standard single mode communication fiber (SMF-28e) with a short unjacketed section was placed in two fiber holders. Both ends of the fiber were clamped by the holders and held straight. Two electrodes were mounted in a fixture that was moved by a nanoprecision translation stage. The grating spectrum was monitored while the arc discharge was being produced. The entire fabrication process was fully controlled by a computer. Two methods have been used to fabricate LPFGs by electric arc discharge. The first creates micro-bends by introducing a small lateral displacement at one end of the fiber. The other tapers the fiber by attaching a mass to one end. Both methods induce refractive index modulation along with mechanical deformation. Depending on the position of the fiber in the arc flame and the applied stress to the fiber, the induced refractive index profile inside the fiber can be greatly affected, which in turn, affects the coupling constant between core and cladding modes. For our experiment, the fiber sat in V-grooves made on the electrodes fixture. There was no lateral displacement at one end of the fiber, and no additional mass was attached. The arc current was $\sim 15 \mathrm{~mA}$ (RMS) with $20 \mathrm{kHz}$ frequency, and the arc duration was changed while keeping arc current constant.

Figure 1.22 shows the evolution of a short LPFG as the number of arc discharges is increased. The arc duration was $357 \mathrm{~ms}$, and the period was $500 \mu \mathrm{m}$ for this grating. The resonant coupling rapidly grew and reached its deepest peak $(-30 \mathrm{~dB})$ with 4 grating periods ( 5 arc discharge). The peak depth was reduced to $-13.2 \mathrm{~dB}$ with one more arc discharge due to over-coupling. The total grating length was $2 \mathrm{~mm}$ when it reached the deepest peak. Using the measured peak depths, the number of periods, and the relation $t_{x, \max }=\sin ^{2}(\kappa L)$, the coupling constant for this mode was estimated to be in the order of 10 $\mathrm{cm}^{-1}$, which is over ten times larger than the typical coupling constant of LPFGs. 


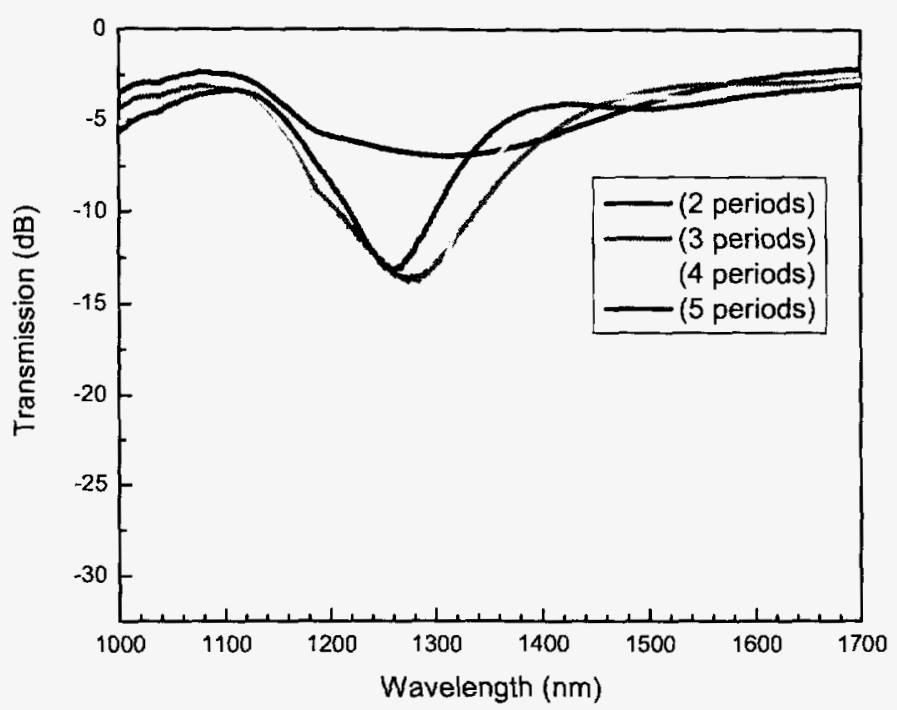

Fig. 1.22. Evolution of the short arc-written LPFG.

For this short grating, a mechanical deformation was observed by an optical microscope as shown in Fig. 23. The magnitude of deformation was estimated to be approximately $10 \mu \mathrm{m}$.

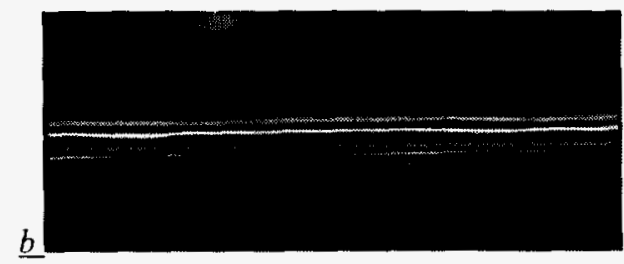

Micro deformation

Fig. 1.23. Optical microscope image of short arc-written LPFG.

\section{The major advantages of our approach are:}

- We have successfully realized multiple gratings (six gratings in a single sapphire fiber), which is the key for the distributed sensing, as shown in Fig. 1.24. 


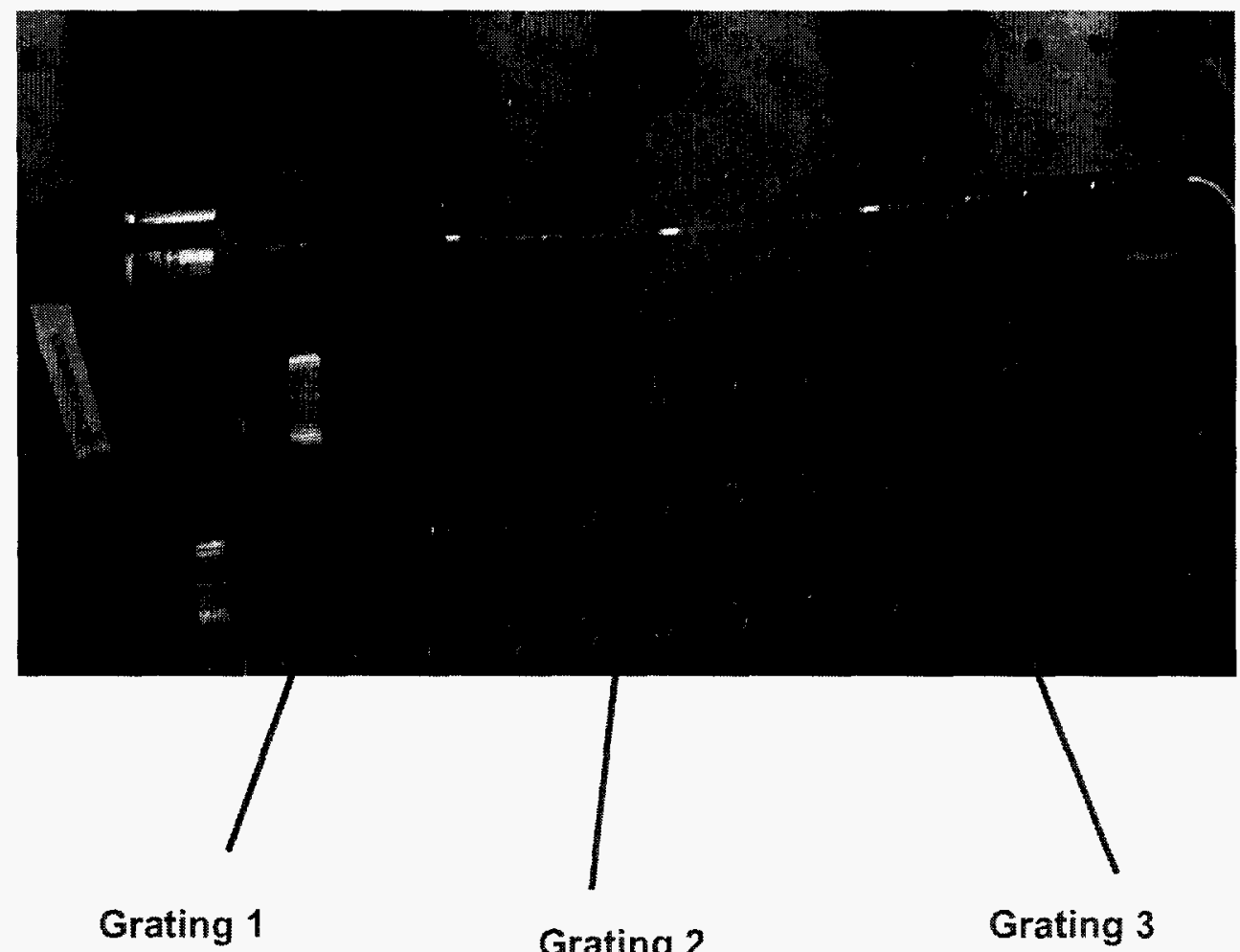

Fig. 1.24. The multiple gratings fabricated in the same sapphire fiber.

These sensors are multiplexed sensors rather than distributed sensors because they don't provide continuous measurement along the fiber. By multiplexing several sensors, however, a few centimeters spatial resolution can be readily obtained, which has enough resolution in most cases. These 'quasi-distributed'sensors have high sensitivities, simple structures.

- We have demonstrated that Femtosecond Bragg gratings are much more stable in high temperature environments than UV induced gratings. We have durability test on the grating. The spectrum is stable except some predictable drifting. Femtosecond laser inscription of Bragg grating in optical fiber is the most promising method to fabricate harsh environment sensors.

- We have developed multi-parameter sensor based on asymmetric multimode fiber Bragg grating fabricated by femtosecond laser irradiation.

\subsection{Related Technical Publications}

$\underline{\text { Refereed Journal Papers }}$ 
[1] Chun Zhan, Jon Lee, Zhu Yong, and Shizhuo Yin, "Photo-enhanced polarization mode separated fiber Bragg gratings inscribed by femtosecond laser", Submitted to Jounal of Applied Physics.

[2] Chun Zhan, Yong Zhu and Shizhuo Yin, "Asymmetric Bragg gratings inscribed by IR femtosecond irradiation for harsh environment multi-parameter sensing applications", To be published in Optical Fiber Technology. (Invited paper)

[3] Zhu Yong, Chun Zhan, Jon Lee, and Shizhuo Yin, "Multiple parameter vector bending and high temperature sensors based on asymmetric multimode fiber Bragg gratings inscribed by infrared femtosecond laser" Opt. Lett. 31, 1794-1796 (2006).

[4] Y. Yang, K. Chung, S. Yin, Z. Liu, and Q. Wang, "Analysis of volume holographic long period grating in photonic nanostructured fibers and waveguides," Optical Engineering, Vol. 43, pp.2003-2008 (2004).

[5] D. Komisarek, K. Reichard, D. Merdes, D. Lysak, P.Lam, S. Wu, and S. Yin, "High-performance nonscanning Fourier-transform spectrometer that uses a Wollaston prism array," Applied Optics, Vol. 43, pp.3983-3988, 2004.

[6] D. Komisarek, K. Reichard, and S. Yin, "Enhancing the performance of nonscanning Fourier transform spectrometer by compensating manufacturing defects inherent to a Wollaston prism array," Optics Communications, Volume 238, pp. 8590 (2004).

[7] Bo Wang, Ruyan Guo, Shizhuo Yin and Francis Yu, "Chemical sensing with Hetero-Core Fiber Specklegram," Journal of Holography and Speckle 1, 53 (2004).

[8] K. Shi, P. Li, S. Yin, and Z. Liu, "Chromatic confocal microscopy using supercontinuum light," Optical Express, Vol. 12, No. 10, pp. 2096-2101 (2004).

[9] Kun-Wook Chung and Shizhuo Yin, "Analysis of a widely tunable long-period grating by use of an ultra thin cladding layer and higher-order cladding mode coupling," Optics Letters, Vol. 29, pp.812-814 (2004).

[10] Kun-wook Chung and Shizhuo Yin, "A highly nonlinear dispersion shifted fiber with $9.3 \mathrm{~mm} 2$ effective area and low loss for all fiber wavelength converter," Microwave and Optical Technology Letters," pp. 153-156 (2004).

\section{$\underline{\text { Refereed Conference Proceedings }}$}

[11] Chun Zhan, Yong Zhu, and Shizhuo Yin, "Fabricating harsh environment fiber Bragg gratings by ultrafast laser pulses”, SPIE 6313-64, San Diego, Aug., 2006

[12] Stuart (Shizhuo) Yin, Sung-Hyun Nam, Yi Yang, Chun Zhan, and Kun-Wook Chung, "Innovative Fiber Optic Gratings: Fabrications and Applications," Proceedings of International Symposium on Advances and Trends in Fiber Optics and Applications, October 11-15, 2004, Chongqing University, Chongqing, China. (Invited Paper)

[13] S. Yin, C. Hahn*, J. Lee*, B. Wang*, and Q. Wang, „Design and implementation of an all-fiber ultra-fast widely tunable wavelength filter (invited)," Proceedings of SPIE on Information Optics and Photonics Technology, SPIE, 5643, pp. *_* (2004) (Principal author; second, third, and fourth authors supervised by candidate) (Invited paper) 
[14] S. Nam, C. Zhun, S. Yin, "High temperature distributed fiber optic sensors," Proceeding s of SPIE on Fiber Optic Sensors, SPIE, 5634, pp. *-* (2004) (First and second authors supervised by candidate) (Invited paper)

[15] W. Su, Y. Hsu, C. Kuo, H. Chan, and S. Yin, "Design and fabrication of digital dual-freuqnecy patterns for projected fringe profilometry, SPIE 5606-20, Philadelphia, PA, Oct. 27, 2004.

[16] S. Yin, B. Wang, C. Luo, Q. Wang, K. Reichard, and D. Lysak, "Development of harsh environment electro-optic polymer and their applications to tunable photonic devices," IEEE LEOS Summer Topic meeting on Optical Interconnects \& VLSI Photonics, MB4.2, San Diego, CA, June 28, 2004.

[17] S. Nam, Chun Zhan, and S. Yin, "Recent advances on fabricating in-fiber gratings in single crystal sapphire fiber," SPIE 5560, pp.147-155, Denver, CO, Aug., 2004.

[18] Jon Lee, B. Wang, C. Hahn, K. Reichard, D. Ditto, D. Glista, Q. Wang, and S. Yin, "Harsh environment electro-optic polymer and its application to in-fiber tunable filter," SPIE 5560, pp. 52-63, Denver, CO, Aug., 2004.

[19] B. Wang, Y. Yang, Y. Liu, S. Yin, R. Guo, P. Ruffin, "Fiber optic sensors using photonic crystal fiber embedded heterofiber structures," SPIE 5560, pp.284-294, Denver, CO, Aug., 2004.

[20] Wei-Hung Su, Cho-Yo Kuo, and Shizhuo Yin, "Design and fabrication of various digital fringe patterns for projected fringe profilometry," SPIE 5560, pp. 193-203, Denver, CO, Aug., 2004.

[21] K. Reichard, S. Yin, C. Hahn, J. Lee, B. Wang and Q. Wang, "An athermal design for an all-fiber, ultra fast, widely tunable wavelength filter," Avionics, Fiber-Optics and Photonics Workshop, Workshop Notes, ThP15, St. Louis Marriott Downtown, St. Louis, MO, 21-23 April 2004.

[22] S. Yin, S. Nam, P. Ruffin, K. Reichard, "An investigation of a photonic bandgap based gyroscope and its application to Avionics, Avionics, Fiber-Optics and Photonics Workshop, Workshop Notes, ThP17, St. Louis Marriott Downtown, St. Louis, MO, 21-23 April 2004.

[23] Sung-Hyun Nam, Jesus Chavez, and Shizhuo Yin, "Fabricating in fiber gratings in single crystal sapphire fibers," SPIE 5350, pp.58-65, San Jose, CA, Jan., 2004.

[24] Shizhuo Yin, Thomas W. Gardner*, Fei Wu and Milind Cholker, "Optical combing to align photoreceptors in detached retinas," SPIE 5314, pp.298-305, San Jose, CA, Jan. 2004.

[25] Yi Yang, KunWook Chung, Shizhuo Yin, Karl Reichard*, Zhiwen Liu, and Qing Wang+, "Highly sensitive sensors using in-fiber gratings fabricated in photonic crystal nanostructures," SPIE 5360, pp.237-246, San Jose, CA, Jan., 2004. 


\section{TASK 2. BOILER FURNACE MONITORING MODEL DEVELOPMENT}

\subsection{Summary}

In the first year, the effort was primarily to get a functional workstation, with CFD software, into place and to train the student on its application to multi-dimensional combustion simulation. The workstation acquired for this project comprises dual-Xeon $2.0 \mathrm{GHz}$ processors and $512 \mathrm{MB}$ RAM. A license for FLUENT 6.1 was been obtained. The graduate student was trained with FLUENT to achieve the goals of this project with regard to multi-dimensional simulation.

In the second year, we are now working with a 2-D model of the Down Fired Combustor and a 3-D model of the Demonstration Boiler.. Modeling the DFC has provided the graduate student the opportunity to become skilled using FLUENT, to leverage existing grids and extensive prior experimental work for comparison. The output from the DFC modeling will be temperature maps for use in training the predictive tool to describe the temperature distribution within the combustor to effect control actions.

In the $3^{\text {rd }}$ year, subsequent applications of FLUENT to the Demonstration Boiler focused on determining the means by which the output from the fiber optic sensor could be used to determine whether some control action is needed to reduce emissions (i.e., NOx). Of particular interest was seeing how the signals from one or more of the fiber optic sensors can tell us how the temperature field needs to be modified, via for example some adjustment of air staging distribution, to obtain lower NOx emissions. The detailed simulations were intended to provide guidance on how to achieve the intelligent control over efficiency and emissions.

\subsection{Results and Discussion}

\subsubsection{Group A}

Temperature profiles of $2 \mathrm{D}$ planes throughout the boiler were examined from the final calculations; examples of such profiles are shown in Fig. 2.1 and Fig. 2.2. These figures show that stratification of temperature within the boiler varies for case 1 and case 2, therefore lending credence to the notion that temperature signatures within the boiler are unique, and may relate to NOx emissions at the boiler exit.

Note the dark blue portion of the profile in Fig. 2.2; this low temperature area near the burner may be indicative of flame instability. The high volume of tertiary air, therefore removing air volume from the secondary inlet, may have resulted flame stability issues.

Temperature and NOx data along 1D lines of site were extracted from the combustion calculations; several 1D profiles of temperature and NOx are shown in Fig. 2.3 and Fig. 2.4 . 


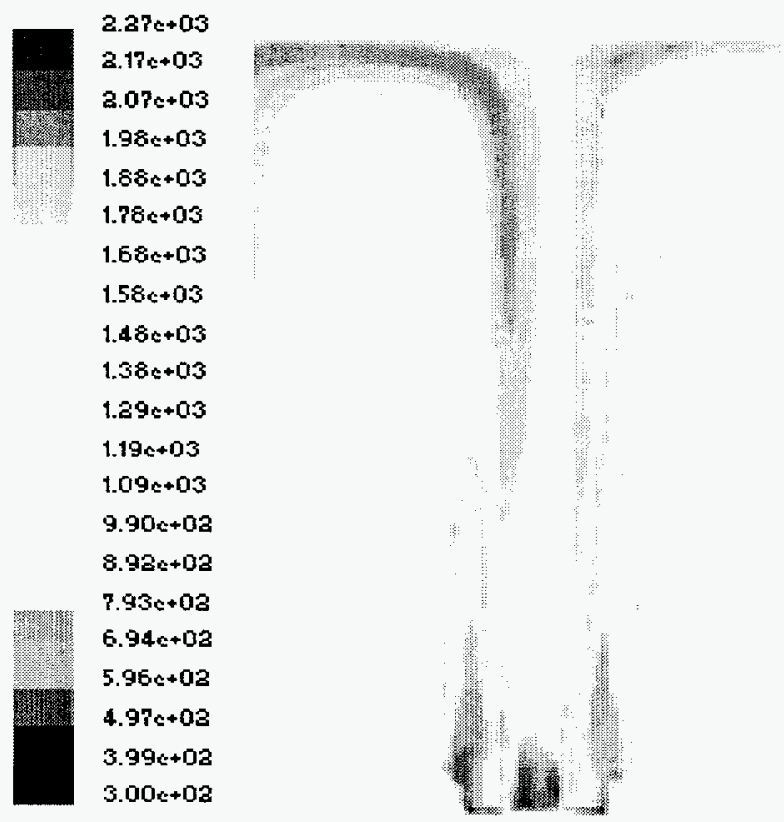

Fig. 2.1: Temperature Profile (horizontal) - Case 1
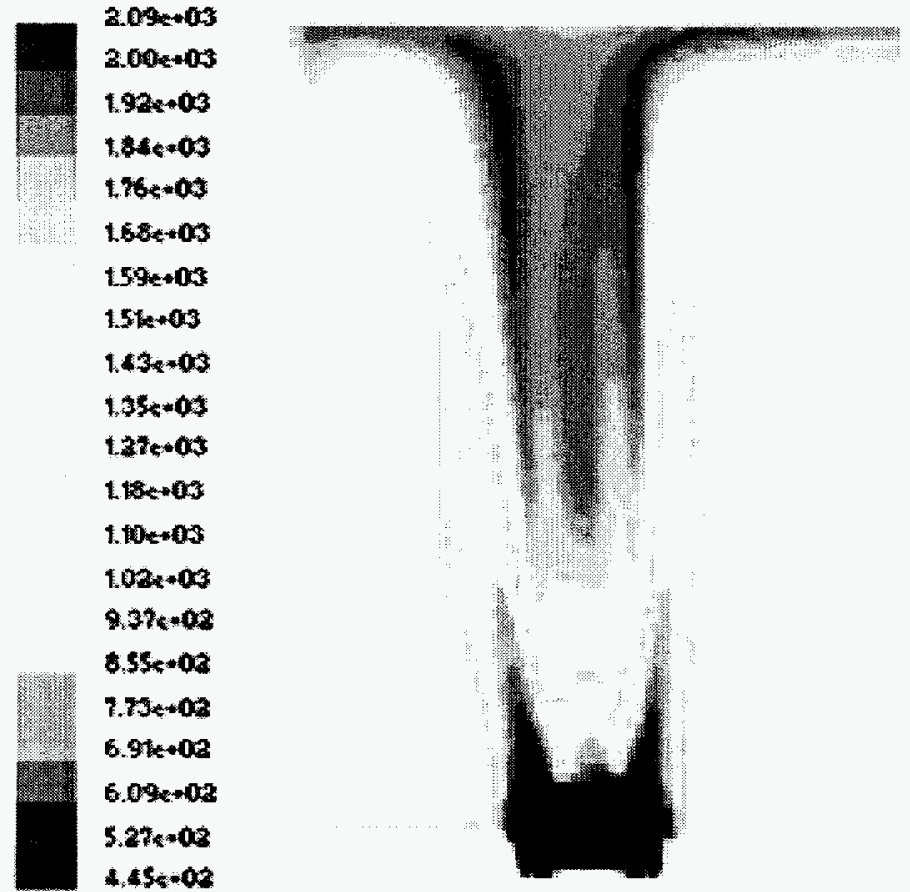

Fig. 2.2: Temperature Profile (horizontal) - Case 2 


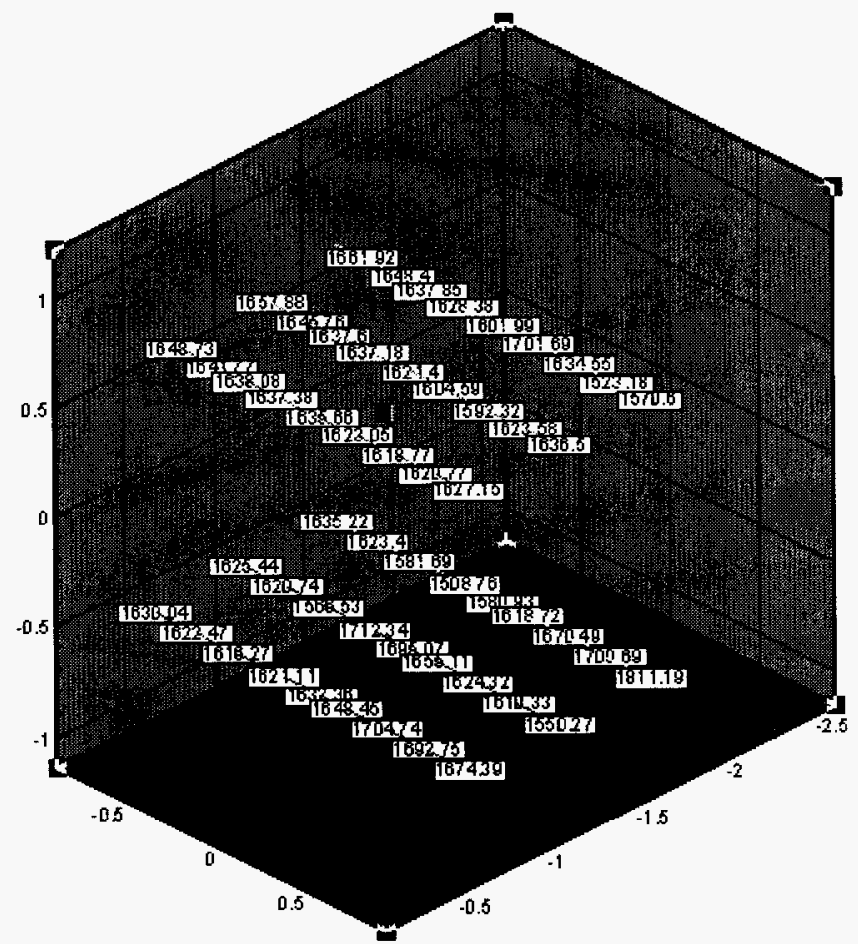

Fig. 2.3: In-situ Temperature (K) Distributions - Case 2

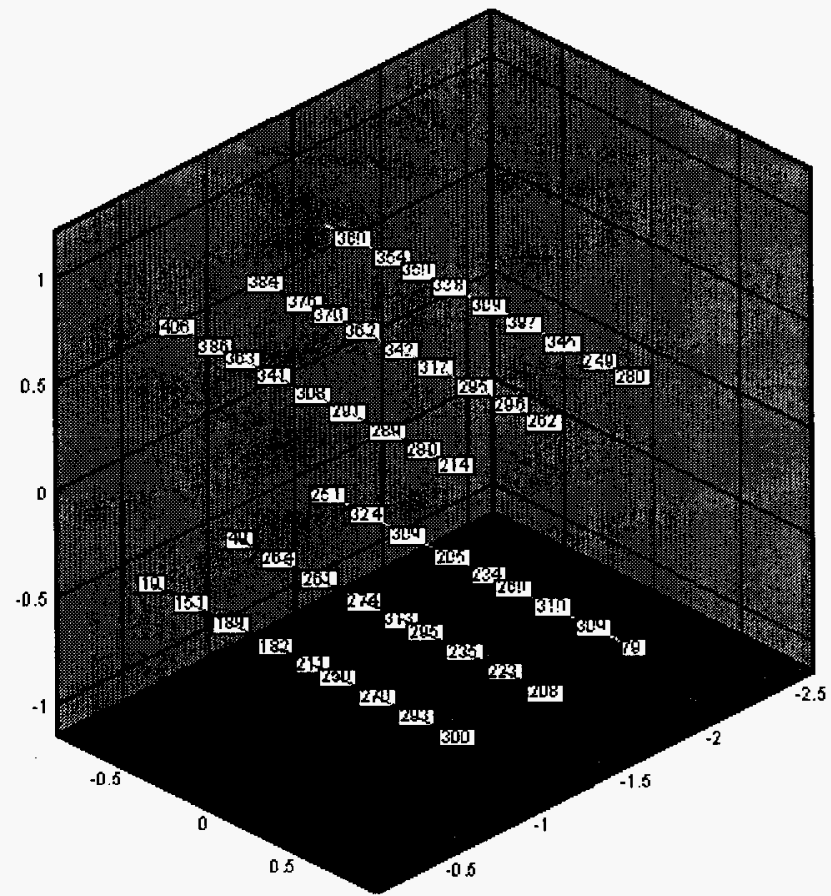

Fig. 2.4: In-situ NOx (ppm) Profiles - Case 2 
The exit NOx concentrations were a key part of the investigation. Table 2.1 shows the summary of NOx concentrations and temperatures at the boiler exit for each case. Note that as the amount of tertiary air is increased, the NOx exiting the system decreases (also note that lower exit temperatures correspond to lower exit NOx). Statistical tests were conducted using SAS/STAT ORTHOREG procedure for temperatures extracted along the lines of site within the boiler, and the corresponding NOx emissions calculated at those points; the series of tests are outlined in the appendices. Statistical test \#1 yielded the highest R-squared value of 0.84 , and provided the following relationship between NOx (internal) and temperature (K). The results of the statistical analysis are compared against the raw data (NOx), as a function of data point (the 12 sensors comprised a total of 904 data points), are shown in Fig. 2.5, Fig. 2.6 and Fig. 2.7.

$$
\mathrm{NOx}(\mathrm{ppm})=\left[1.48 \mathrm{E}^{-4}-4.34 \mathrm{E}^{-7} * \mathrm{~T}+3.37 \mathrm{E}^{-10 *} \mathrm{~T}^{2}\right]^{*} 10 \mathrm{E}^{6}
$$

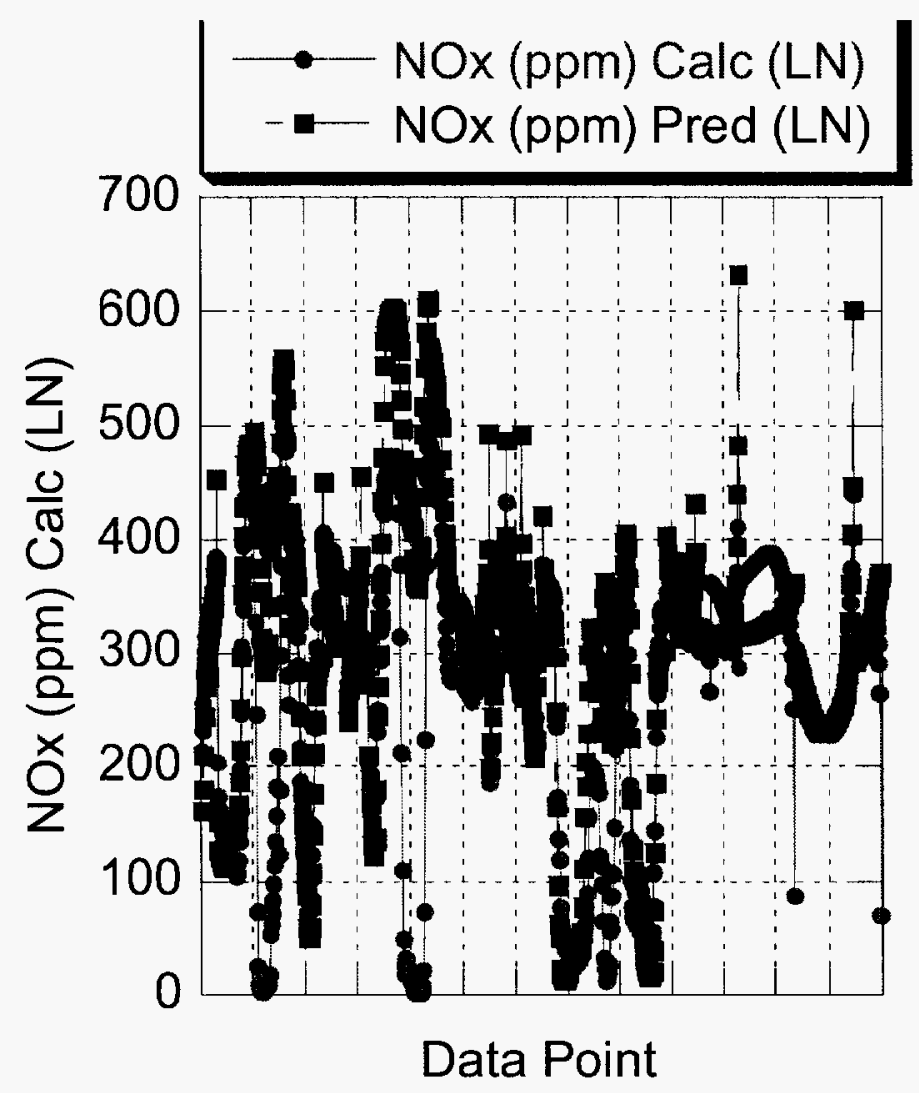

Fig. 2.5: NOx (ppm) Measured and Predicted - Case 1: Group A 


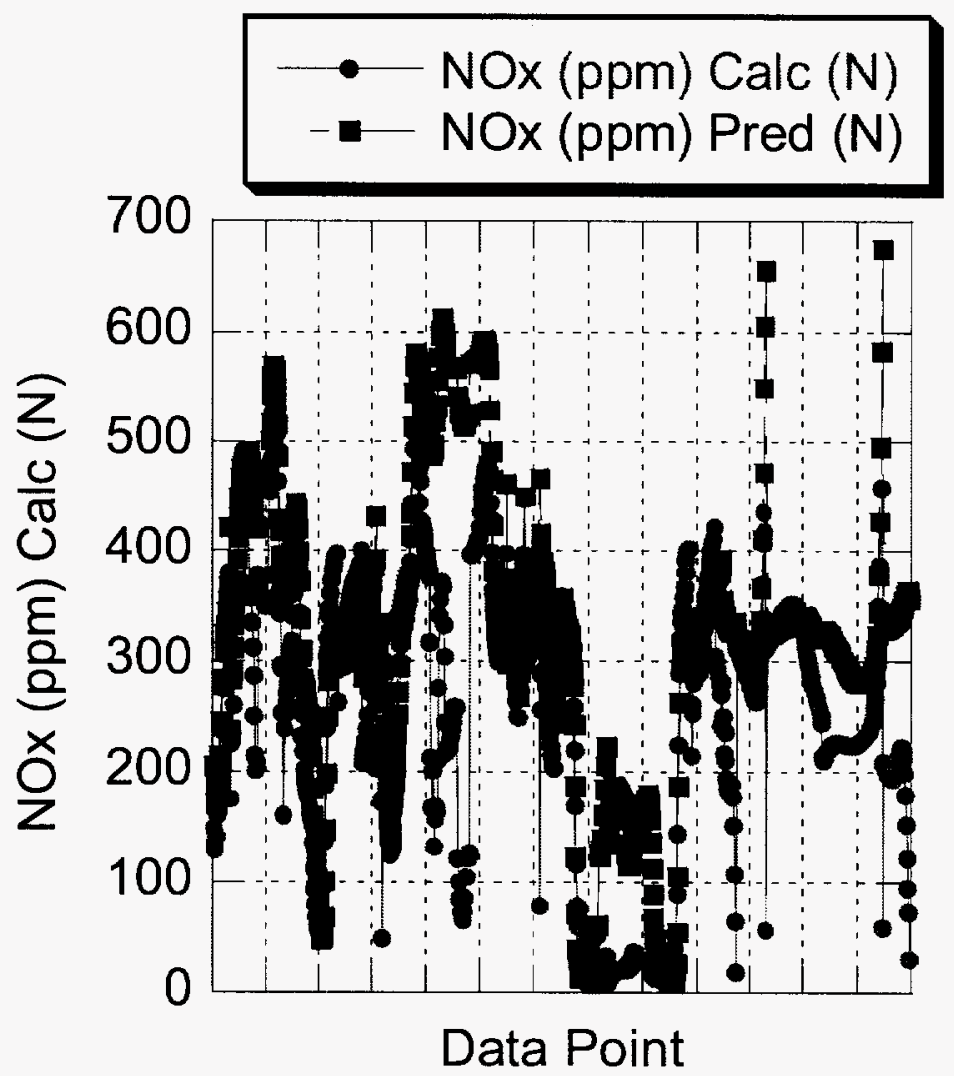

Fig. 2.6: NOx (ppm) Measured and Predicted - Case 2: Group A 


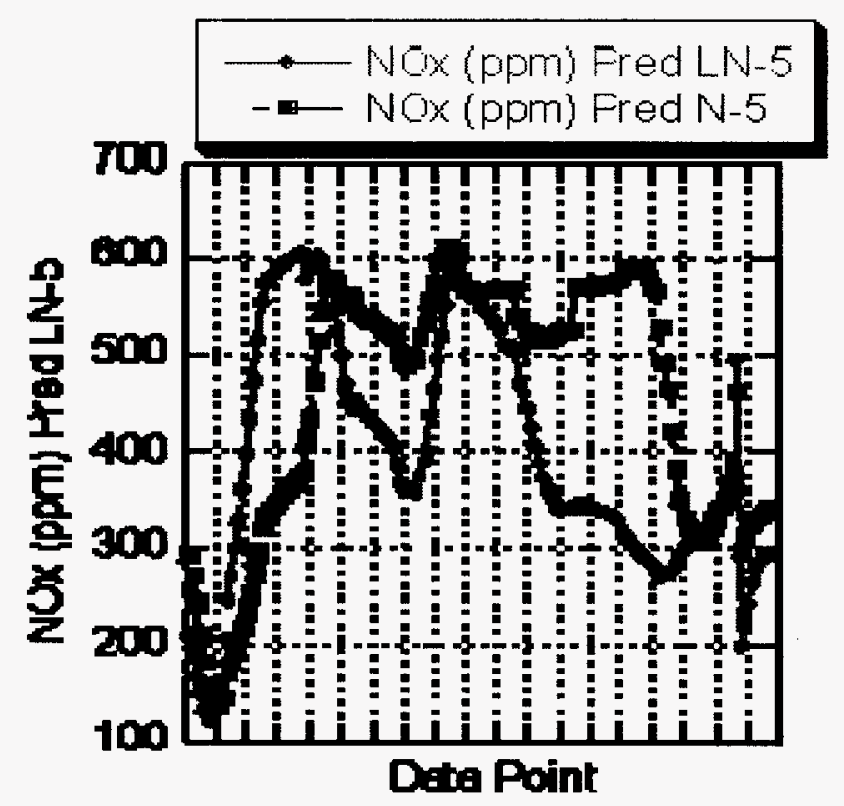

Fig.2.7: NOx (ppm) Prediction Comparison: Group A \& B

The relationship in Equation 2.1 allows one to determine NOx concentrations at points along 1D lines of site within the boiler, for temperatures measured along those lines of site (with an optical sensor). While this relationship is rather strong, relates back to the research performed by Fiveland and Latham as it shows evidence of significant stratification, it provides no relationship between the temperature within the boiler and the temperature at the boiler exit (Fiveland and Latham 1993). Without such a relationship, the novel temperature sensor is futile. The temperature stratification information along with the difference in boiler exit NOx concentrations for both cases, however, indicates that exit NOx concentrations may be correlated with temperature within the boiler somehow.

Only two cases were examined in this study, and therefore it is impossible to determine a definitive correlation between temperature within the boiler and NOx concentration at the exit.

The species selected for the PDF in the combustion model could have been improved. Species such as $\mathrm{C}_{3} \mathrm{H}_{8}$ could have been implemented. Also, a two-stream PDF could have been used to account for the char streams and volatile streams individually, improving the accuracy of the calculations.

The Group A calculations converged near 15,000 iterations, based on convergence criteria outlined in the FLUENT manual. However, the residual for the energy equation was raised to $10^{4}$, which may prove a source of error for the above calculations, as the residual was altered during the calculation.

\subsubsection{Group B}


Group B comprises four cases at different operating conditions. The parameters and models selected for these cases are consistent for each case, except for the ratios of secondary and tertiary air. Aside from improved model selections, the difference between Group B and Group A cases is the swirl applied to the tertiary air in Group B calculations. Data was extracted from 12, 1D sensor locations throughout the boiler (the same sensor locations as in Group A calculations) to be used for statistical analysis to determine a relationship between in-situ temperature measurements and exit NOx concentrations Table 2.2 shows the various air ratios between tests, as well as the NOx concentrations and temperatures at the boiler exit. Note that for these calculations, as the amount of tertiary air increases, the amount of NOx decreases, which follows the trend from Group A computations.

Table 2.2: Boiler Outlet Conditions: Group B

\begin{tabular}{|lcccc|}
\hline & Case 1 & Case 2 & Case 3 & Case 4 \\
\cline { 2 - 5 } Primary Air & 0.200 & 0.200 & 0.200 & 0.200 \\
Secondary Air & 0.200 & 0.375 & 0.550 & 0.725 \\
Tertiary Air (swirled) & 1.590 & 1.415 & 1.240 & 1.065 \\
Fuel Flow Rate (kg/s) & 0.17 & 0.17 & 0.17 & 0.17 \\
$T_{\text {exit }}(\mathrm{K})$ & 1441 & 1523 & 1554 & 1617 \\
NOx $_{\text {exit }}(\mathrm{ppm})$ & 164 & 527 & 837 & 924 \\
\hline
\end{tabular}

In attempt to find a numerical correlation between temperature profiles within the boiler and exit NOx concentrations, or to provide proof of unique temperature profiles for each case, many relationships were studied. The first test involved running a series of tests in SAS/STAT with the ORTHOREG procedure. The series of tests are outlined in the appendices, and resulted in poor relationships, with R-squared values ranging from 0.01 to 0.6 . Due to undesirable Rsquared values, the relationships from these tests were dismissed. The statistical tests prove that there is no linear relationship between temperature and NOx along the 1D sensors within the boilers.

In order to determine for sure whether any unique relationships between temperature(s) within the boiler and exit NOx exist, a series of statistical tests were completed. The results are shown graphically in the following section, and tabulated in the appendices. Note that the concentration of NOx at the boiler exit is as follows for Group B simulation cases: Case $1<$ Case $2<$ Case $3<$ Case 4.

Though the in-situ boiler temperature measurements seem to provide no direct relationship with NOx at the boiler exit, temperature at the boiler exit and the corresponding NOx concentrations were used in conjunction with air ratios for further statistical tests. For each case from the Group $\mathrm{B}$ calculations, area-averaged temperatures and NOx values from the boiler exit and tertiary air values were used to form a linear regression study in MiniTab. The results are shown in Equation 2.2, and show that exit temperatures and tertiary airflow values have a strong ability to predict exit NOx concentrations. In this equation, "Air" represents the amount of tertiary air entering the boiler, and " $\mathrm{T}$ " is temperature. 


$$
\begin{aligned}
& \text { NOx }(\mathrm{ppm})=-68+1.35 \mathrm{~T}-1050 \mathrm{Air} \\
& \text { R-squared }=94.5 \%
\end{aligned}
$$

The above regression study was repeated using the primary air values and the tertiary air values. This case reported the same regression equation and R-squared value. When substituting the values for secondary air for the tertiary air, a similar regression equation was reported, with an identical R-squared value. A regression study of NOx, T, secondary and tertiary air was also attempted, however, the similarity in changes in values between cases for secondary and tertiary values were too close, MiniTab prompted that one set of air values be eliminated.

Figure 2.8 depicts a plot of the actual NOx concentrations at the boiler exit, from simulations, as a function of temperature at the boiler exit, as well as the same trend achieved using the relationship from Equation 2.2. As the r-squared value of that equation indicates, the predicted trend relates closely to the simulated data.

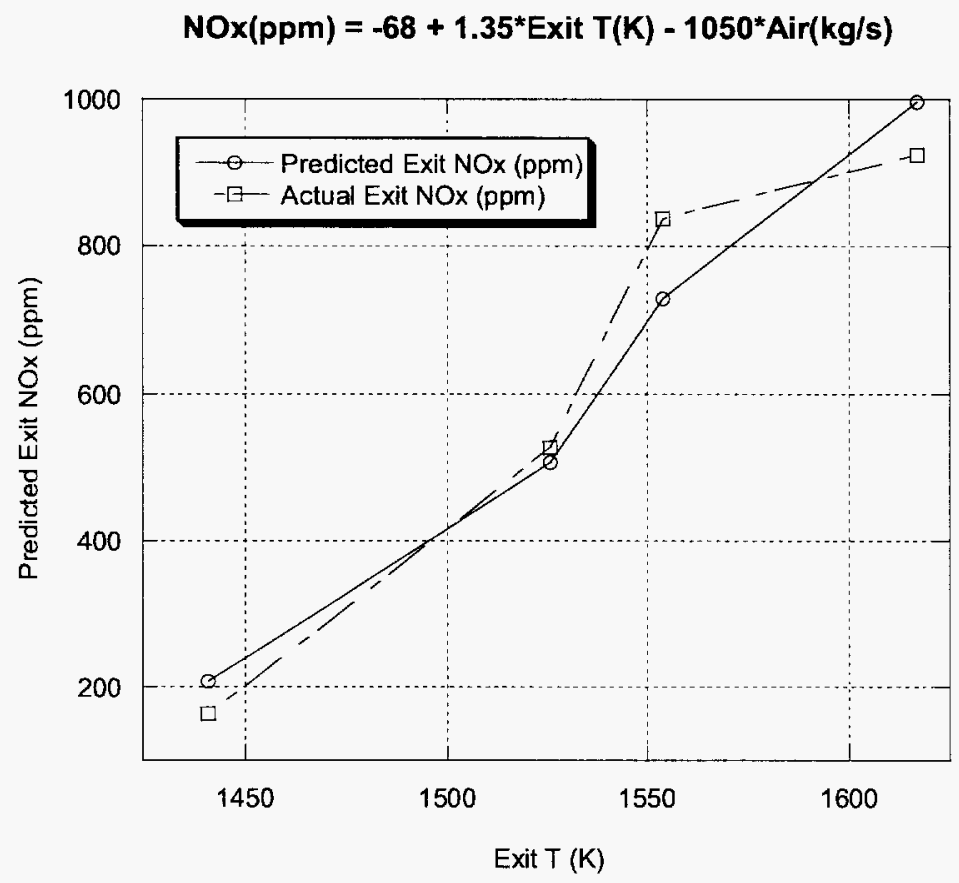

Fig. 2.8: Exit NOx (ppm) Measured and Predicted (from Equation 4.2)

The PDF in Group B included $\mathrm{C}_{3} \mathrm{H}_{8}$, but did not utilize the two-stream functionality. As mentioned in a previous section, the two-stream functionality allows for handling of the char streams and volatile streams individually, instead of lumping them together as one entity, therefore increasing the accuracy of the calculation. The computation time associated with the two-stream model is significantly greater than for the single stream PDF, and therefore the single-steam function was employed. 
Residuals for temperature, $\mathrm{O}_{2}$ and $\mathrm{CO}_{2}$ at the boiler exit were monitored, and the calculations were considered converged when the fluctuation in residual values become consistent over a period of 5,000-10,000 iterations. Data was then extracted where $\mathrm{O}_{2}$ values were minimal and $\mathrm{CO}_{2}$ concentrations were at a maximum, within the range of consistent fluctuations. The fluctuating residuals can be linked to back flow at the boiler exit. Due to the turbulent nature of combustion, especially with swirled inlet flows in an industrial sized boiler, it is not likely that the fluctuations would ever level out. One simulation ran for 48,000 iterations, and the fluctuations remained consistent.

\subsection{Group A \& B Comparison}

The statistical analysis of Group A and Group B data are compared graphically, based on the following parameters:

$$
\begin{aligned}
& T_{\text {avg }} \quad \text { Average of temperatures from one sensor } \\
& \Delta T_{\text {avg }} \quad \text { The absolute difference between temperature values along } \\
& \\
& \begin{array}{l}
\text { one sensor, averaged over the number of differences } \\
\text { calculated }
\end{array}
\end{aligned}
$$

Median The middle number of a group of number arranged

in ascending order

Std.Dev. Standard deviation is the average amount by which

values in a distribution differ from the mean value

of that distribution

Variance The square of the standard deviation value

The goal of comparing the values explained above is to discover unique trends between cases in one data group (Group A or Group B), between temperature data from within the boiler and exit NOx concentrations.

Figure 2.9 and Figure 2.10 show $\mathrm{T}_{\text {avg }}$ values of the data for each sensor, from each case studied. Note that the data for each case in Group A follows a similar trend, with this exception of the data at sensors \#1 and \#2. Sensor \#1 spans the boiler from the burner to the back of the boiler, throughout the boiler center. Recall that the temperature profile for Case 2 shows a significant low temperature area near the burner region. Each case in Group B studies follows a consistent trend, with variations in temperature averages.

The data plotted in Figure 2.11 and Figure 2.12 shows that the trends for each case within each data group are very similar. The data for sensor \#3 in the Group B data, however, shows some variation. Sensor three is location in the region near the top of the boiler. 


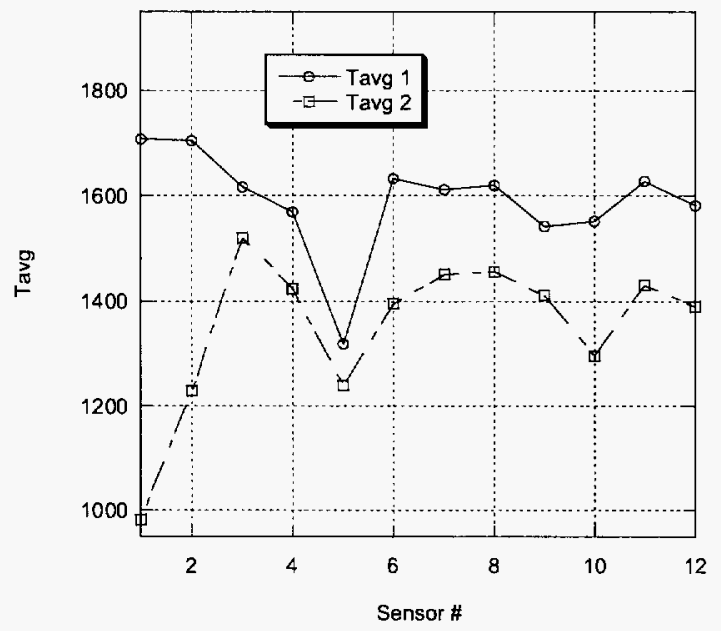

Fig. 2.9: $\mathrm{T}_{\text {avg: }}$ Group A

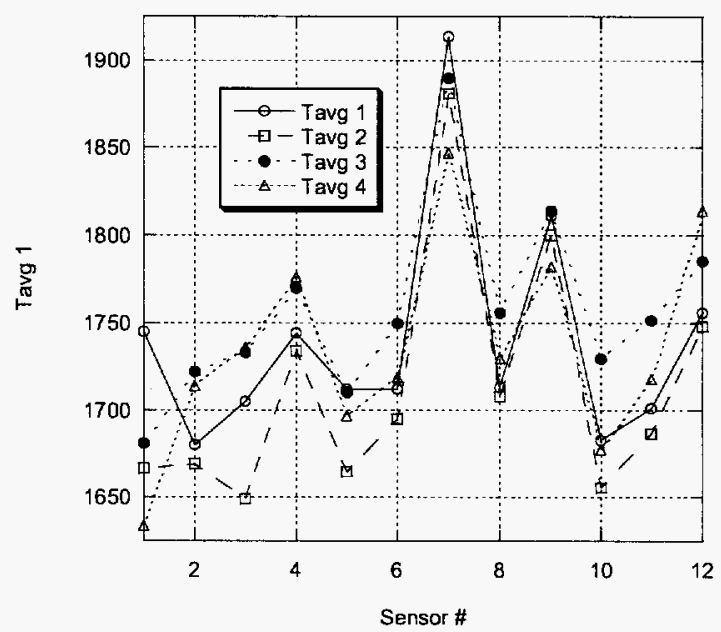

Fig. 2.10: $\mathrm{T}_{\text {avg: }}$ Group B

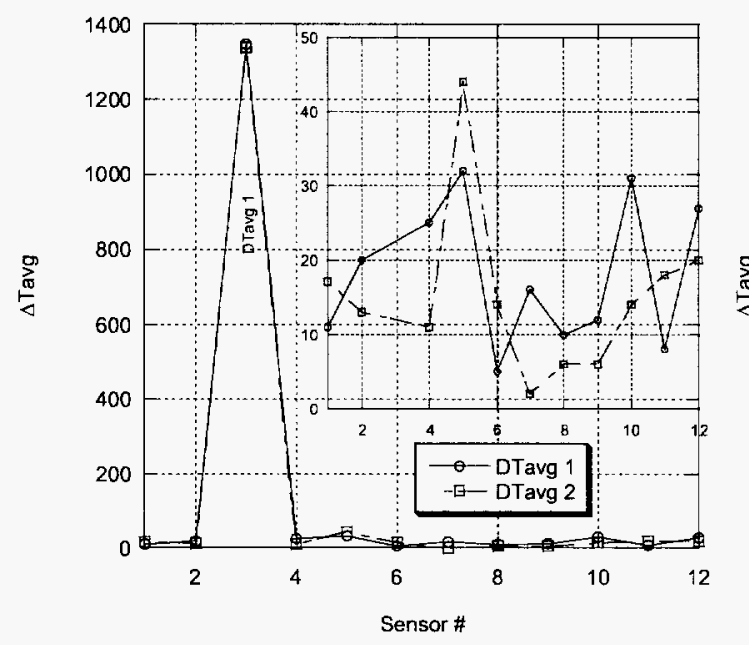

Fig. 2.11: $\Delta \mathrm{T}_{\text {avg }}$ : Group A

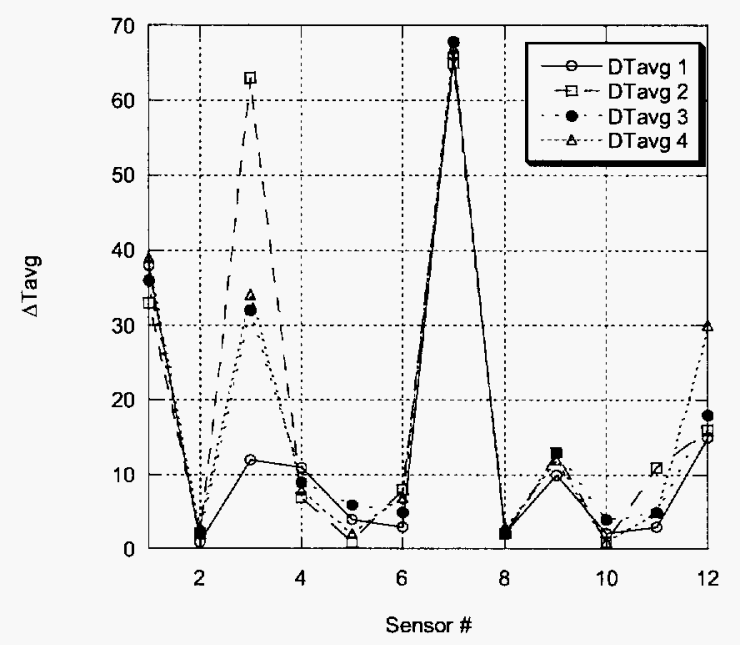

Fig. 2.12: $\Delta \mathrm{T}_{\text {avg }}$ : Group B

The trends generated from the median temperature of each sensor are shown in Figure 2.13 and Figure 2.14. The data for Group A shows a unique trend for each case; but this is not true for Group B data; therefore, the relationship of median temperature to exit NOx concentration is not robust enough to predict relationships for a wide range of cases. [Again, note that the values at sensor \#1 and sensor \#2 are out of place for the Case 2 curve, as explained before due to the low temperature region in the boiler]. 


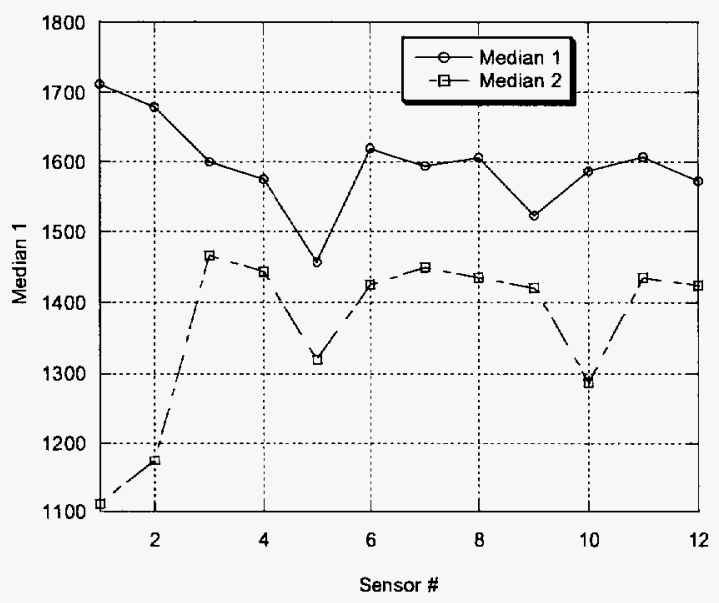

Fig. 2.13: Median: Group A

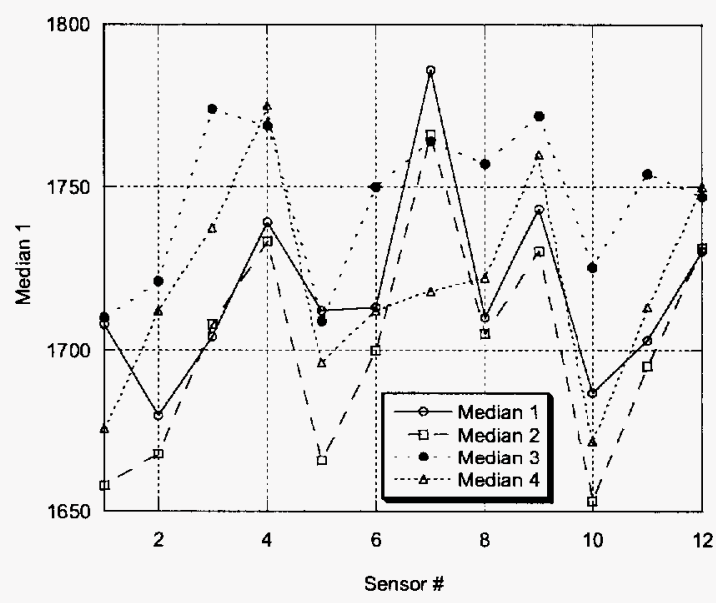

Fig. 2.14: Median: Group B

Standard deviation and variance were also examined for data sets from each sensor, in hopes that the variation of temperature from one spatial location to the next may provide unique information in terms of temperature for each case. This idea was inspired by the work of Fiveland and Latham, and Epple, where temperature signatures for several cases were shown to be unique, as a result of air staging using low-NOx burners (Fiveland and Latham, 1993, Epple, et al. 1995). Figure 2.15 and Figure 2.16 show the standard deviation trends, and Figure 2.17 and Figure 2.18 contain variance plots. None of the curves produced for variance or standard deviation provide unique curves compared to the others within their data group (Group A or Group B).

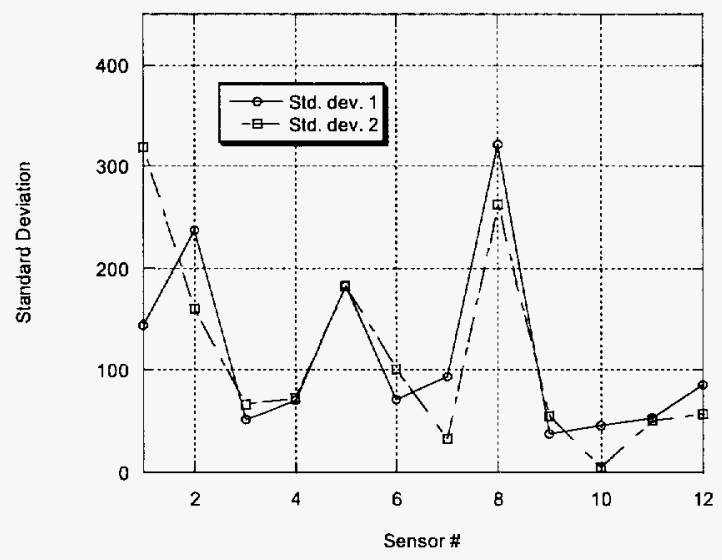

Fig. 2.15: Standard Deviation: Group A

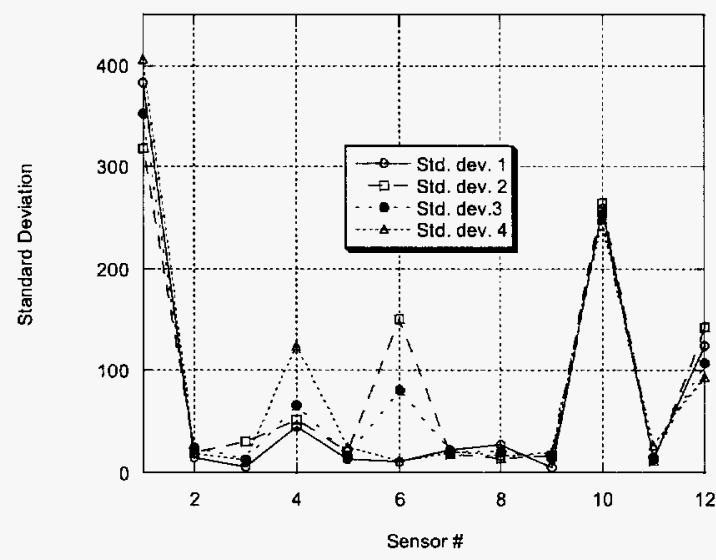

Fig. 2.16: Standard Deviation: Group B 
Because attempts to relate in-situ temperature profiles with exit NOx concentrations proved futile, relationships between the temperature at the exit, air ratios (entering the boiler), and exit NOx concentrations were examined. Figure 2.19 shows a plot of the exit NOx concentration as a function of temperature for each simulated case, as well as a prediction of exit NOx concentration from a relationship derived from the actual data, using temperature and NOx concentration at the boiler exit as well as tertiary air flow rate. This prediction provides unique exit NOx concentrations for each case. The data labels represented the Group (A or B) and the Case (1-4). Figure 2.20 shows the predictions from the equation derived using exit NOx concentrations, exit temperatures, and the $\%$ tertiary air $(\%$ tertiary air is the ratio of tertiary air to the overall combustion air flow rate entering the burner). The predictions in this study are not unique for all cases modeled.

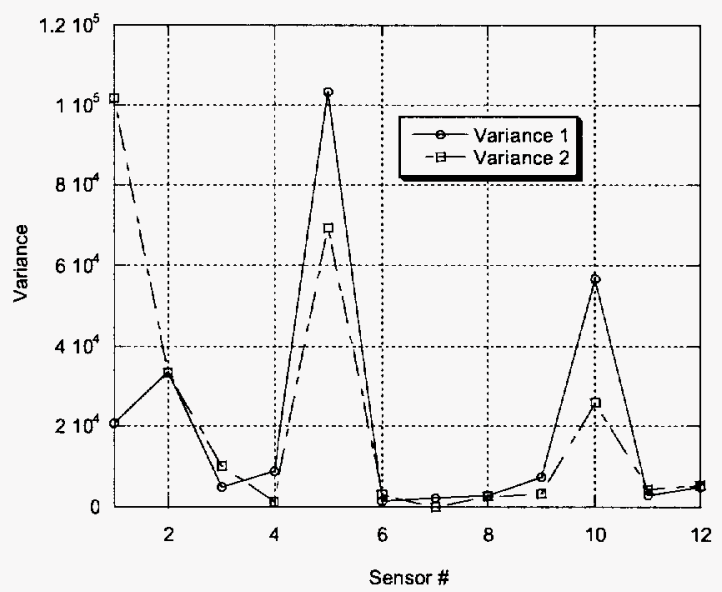

Fig. 2.17: Variance: Group A

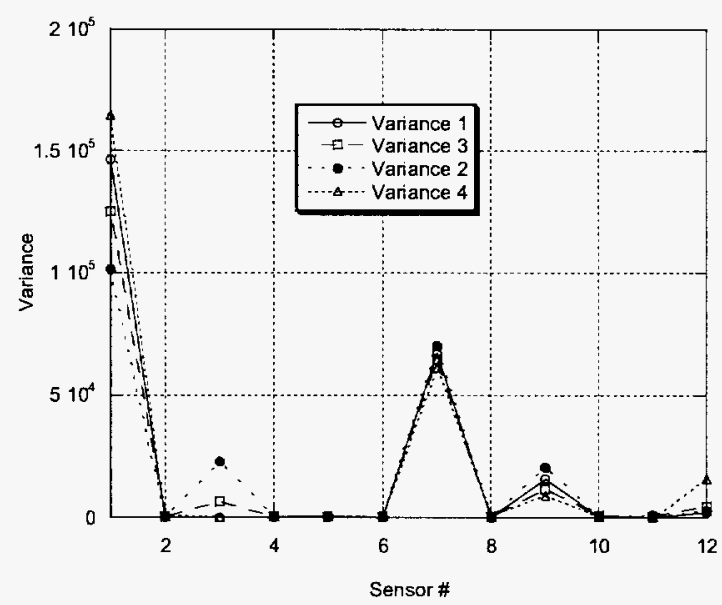

Fig. 2.18: Variance: Group B 


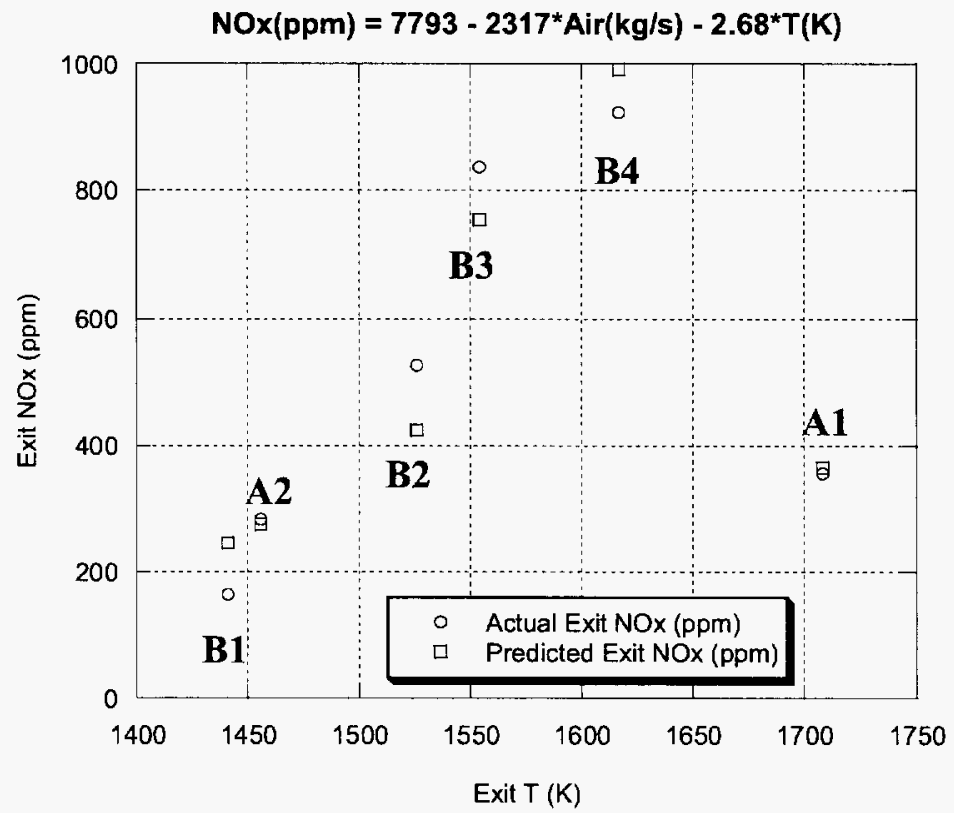

Fig. 2.19: Exit NOx Predictions: Tertiary Air Flow Rate - Group A \& Group B

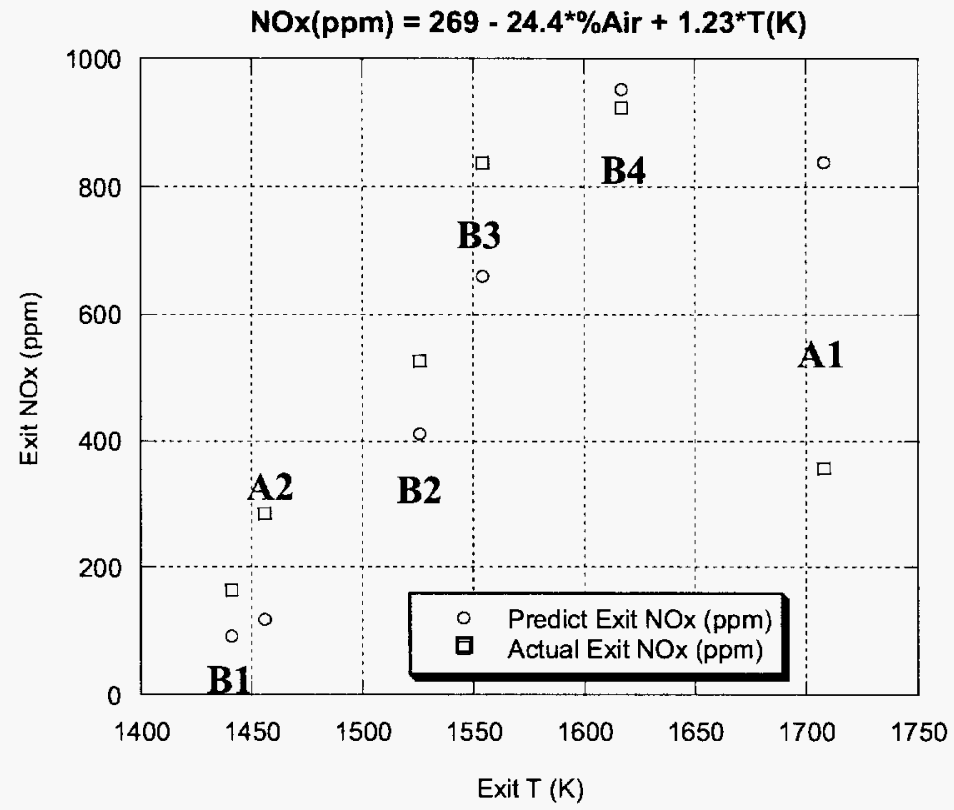

Fig. 2.20: Exit NOx Predictions: \% Tertiary Air - Group A \& Group B 


\subsection{Model Validation}

The computational grid modeled in this study could not be validated with experimental data from the industrial-sized Demonstration Boiler, as the boiler operation of the actual unit varies significantly from the computational model.

\subsection{References}

Epple, B. Schnell, U., Hein, K. (1995). Computerized Analysis of Low-NOx Coal-fired Utility Boilers. Combustion Science and Technology 108: 383-401.

Fiveland, W. A., Latham, C. E. (1993). "Use of Numerical Modeling in the Deisng of Low-NOx Burner for Utility Boilers." Combustion Science and Technology 93: 53-72. 
TASK 3. INTELLIGENT MONITORING SYSTEM DEVELOPMENT

\subsection{Objectives and Motivations}

The proposed project will focus on an investigation of a mathematical approach to extrapolation, using a combination of system-type neural network architecture and the semigroup theory. The target of the investigation will be a class of distributed parameter systems for which, because of their complexity, lack an analytic description. Although the primary objective is extrapolation, this effort must begin with the development of an analytic description from the given empirical data, and then, proceed to extend that analytic description into an adjoining domain space for which there is neither data nor a model. That is, given a set of empirical data for which there is no analytic description, we first develop an analytic model and then extend that model along a single axis. Semigroup theory provides the basis for the neural network architecture, the neural network operation and also for the extrapolation process. Concerning the architecture, semigroup theory dictates that under certain circumstances, a given composite mapping should be regarded as two families of maps, requiring two separate neural network channels; concerning the operation, semigroup theory requires that the second channel possesses the classic semigroup property of mapping composition; concerning the extrapolation process, semigroup theory requires that the extrapolated elements share the same semigroup property that are possessed by the elements previously formed from the given empirical data. The semigroup theory provides a unified and a powerful tool for the study of differential equations on Banach space, covering system described by ordinary differential equations, partial differential equations, functional differential equations and combinations thereof [26]. For applications to control systems, estimation techniques are often required to compensate for an inadequate amount of data, arising from the unavailability of that data.

In the past, for systems described by ordinary differential equations, various estimation techniques have been developed with the most popular (and successful) ones being based on variations of the Kalman filtering theory. However, as control theory has been expanded to include more complex behavior, such as distributed parameter systems, described by partial differential equations, the estimation problem has taken on a new importance, because now it is necessary to provide estimated data at a great (theoretically infinite) number of points. A need therefore exists for a generalized estimation technique that can be applied to a broad class of nonlinear systems, any one of whose behavior is described by a partial differential equation. Stated very concisely, a need exists for a technique which can begin with a sparse set of data derived from a few discrete points within some continuum in one, two or three dimensional space and which can then develop estimated data at as many points as needed within the continuum, in a manner which is dynamically consistent with the given empirical data points, and additionally, to extrapolate the resulting function into an adjoining region of space for which there is no data.

The modeling technique uses a process referred to as algebraic decomposition to find a particular type of smooth approximating function to the empirical data, namely, one that lends itself to a representation as the product of a coefficient vector and a basis set of functions, where the coefficient vector possesses a semigroup property. Extrapolation involves only the coefficient 
vector and begins by training the semigroup channel neural network to replicate the coefficient vector trajectory, while at the same time acquiring a semigroup property of its own (expressed in weight space). The acquisition of the semigroup property is dynamic, expressing itself as a particular sequence of weight changes. The learning algorithm is new in that the weight convergence is realized recursively, by training the neural network repetitively over successively longer intervals and searching for a second level of convergence. Extrapolation is concerned with discovering the dynamics of the weight change sequence and then autonomously continuing that sequence.

\subsection{Development of Intelligent Monitoring System}

Due to the difficulty and the delay of extracting accurate boiler furnace temperature data from FLUENT, the proposed method is applied to extrapolate enthalpy in a power plant to demonstrate the extrapolation capability as another example [27]. In this example, high temperature extrapolation is performed using proposed method. As a final example, due to the lack of the experiment temperature data, the transient heat transfer temperature extrapolation is demonstrated instead of extrapolation using steady state experiment data set. To obtain the transient heat temperature profile, the experimental furnace is modeled and simulated using MATLAB PDE Tool Box.

\subsubsection{Monitoring of Temperature Distribution in Boiler Furnace}

The electric utility industry is charged to deliver power as inexpensively and as reliably as possible. Meeting these dual obligations has become increasingly difficult over the past 30 years. Environmental and economic concerns pressed the utility industry to develop clean and efficient ways of burning coal and oil. This has required major improvements in instrument, data management, and control of electric power plant components such as boilers. It has become a challenge to measure high temperature distributions of high-pressure liquids, steam, combustion gases, and heat transfer components in extremely adverse power plant environments. Traditional sensors have not exhibited sufficient stability and long-term accuracy without requiring expensive maintenance and recalibration. Additionally, each sensor only provides one reading so that only a limited number of readings are obtained.

Fig. 1.1(b) in Task 1 shows the Penn State down-fired combustor (DFC), which is an advanced pilot-scale furnace designed to evaluate the combustion performance of various fuels (natural gas, coal, coal-water slurry fuel) including emissions monitoring. The combustor has a 20 -inch internal diameter, is $10 \mathrm{feet} \mathrm{high,} \mathrm{and} \mathrm{is} \mathrm{designed} \mathrm{for} \mathrm{a} \mathrm{thermal} \mathrm{input} \mathrm{of} 350,000 \mathrm{Btu} / \mathrm{h}$ (nominal), but this can be varied from 200,000 to $500,000 \mathrm{Btu} / \mathrm{h}$. The proposed boiler furnace-monitoring model addresses the estimation of spatial temperature distribution continuously for any operating condition. 
As an alternative to the above model-based estimation techniques, such as infinite dimensional extended Kalman filtering, an intelligent monitoring scheme will be developed for 3D temperature estimation by using the proposed system-type neural networks [28]. An intelligent algorithm will be developed to adaptively tune the monitoring system in real-time to implement in the experimental boilers. The previous emphasis on the application of computational intelligence for control and diagnostic will be shifted to state estimation and prediction problems.

\subsubsection{Extrapolation of Enthalpy in a Power Plant}

The electric utility industry is confronted with the task of estimating the steam enthalpy at various points in the water-steam cycle in a power plant. The two prominent estimation points are at the boiler, where water is converted into steam, and also in the delivery section which precedes the turbine where the energy of the steam is extracted and converted into mechanical power. It is very important to have functions which are able to accurately describe the correlations between enthalpy and temperature for the water/steam because the enthalpy provides the best description of the energy content for a compressible gas. In the literature, there are numerous works which present mathematical functions of enthalpy vs. temperature, but in many cases they provide insufficient approximation with experimental data or have a good approximation only over a small temperature range [29]. In addition, these methods have no provision for extending (extrapolating) accurate readings into a higher temperature range in which the readings become questionable. In this research, a new method of extrapolating the enthalpy is proposed using a system-type neural network architecture. Essentially, rather than relying on questionable temperature readings to calculate the enthalpy, this method extrapolates a set of reliable enthalpy readings directly.

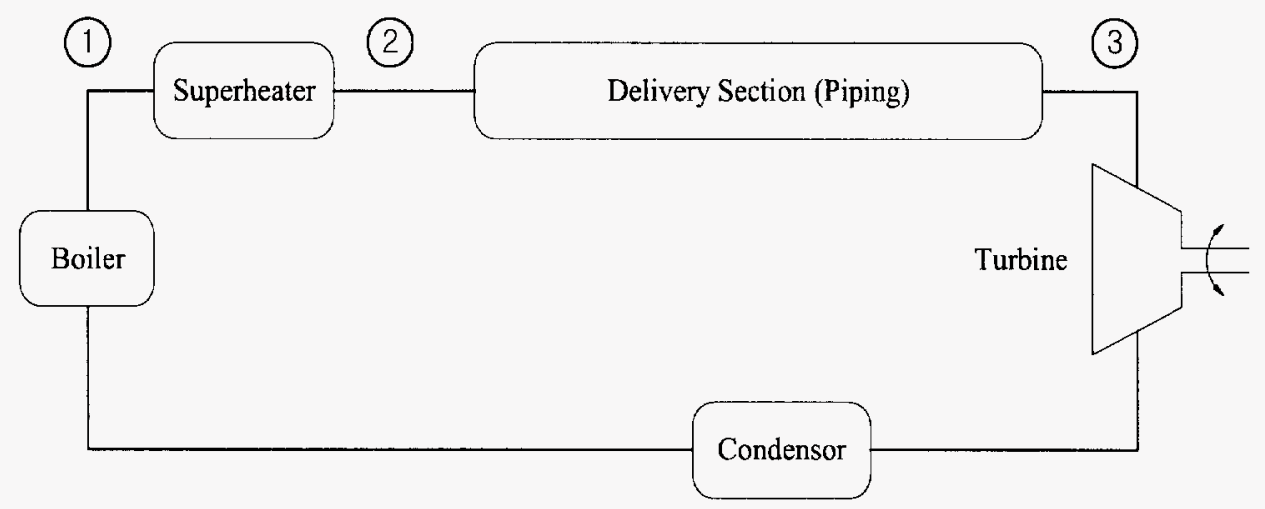

Fig. 3.1. General Power Plant.

Considering the general power plant as shown in Fig. 3.1, and referring to the delivery section from points 2 to 3 , which precedes the turbine in a power plant, there is a need for tracking the steam enthalpy, since it is this function which ultimately determines the mechanical power delivered by the turbine. From the conservation of energy principle, the turbine work per mass of airflow is equal to the change in the enthalpy of the flow from the entrance to the exit of the 
turbine. Therefore, if we can measure the enthalpy among the delivery section and turbine, we can determine the mechanical power which then becomes the electrical power. The difficulty is that the enthalpy is derived from the temperature and, in the usual cases, these involve very high temperatures, and accurate readings of high temperature steam in the presence of high pressures are very difficult to achieve. At present, various temperature compensation schemes are employed but, even with these, the resulting temperature readings are questionable. Therefore, the resulting enthalpy estimations are questionable [30]. The proposed method suggests an alternative, namely, to obtain a small (sparse) set of reliable temperature-pressure readings at the front end of the delivery section, forming the enthalpy from those readings, and then extrapolating those enthalpy readings directly.

\subsubsection{Extrapolation of Transient Heat Transfer}

Due to the lack of the experiment temperature data (only center 5 points along the length are obtained), the transient heat transfer temperature extrapolation is demonstrated instead of extrapolation using steady state experiment data set. To obtain the transient temperature profile, the furnace model is developed and simulated using MATLAB Tool Box. Furthermore, to verify the transient temperature, steady state temperature is also obtained by simulation and is compared with the experimental temperature data set. Fig. 3.2 shows the comparison of experiment temperature and simulation temperature. From this result, it is assumed that the simulation data can replace the experimental data properly.

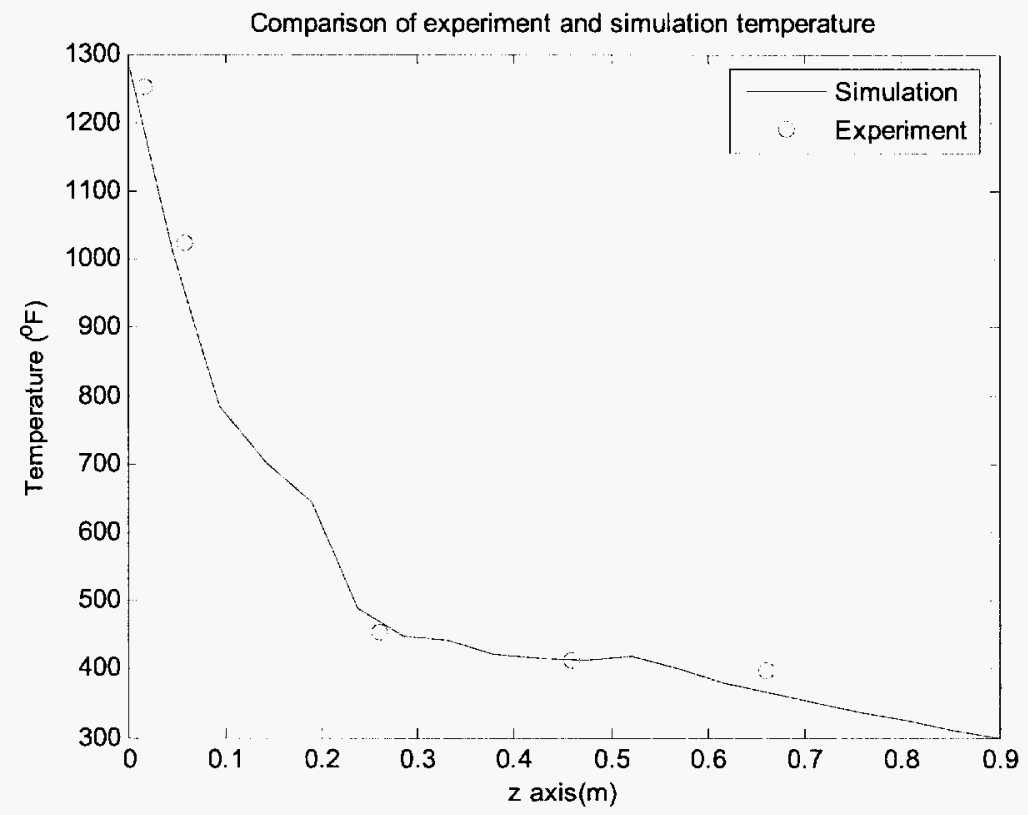

Fig. 3. 2. Comparison of experiment and simulation temperature.

Obviously, many heat transfer problems are often treated as steady ones in practice, but our interest will focus on unsteady or transient state in this section. The steady state is specified as final stage of no change with time at any point in the medium any more, while the transient state implies that there is still some variation with time so far or time-dependent. Therefore, the 
boundary condition of temperature or heat flux remains unchanged at the final state with time to solve a steady problem [47]. Transient heat transfer is that phase of the heating and cooling process when the temperature changes with time. Most of heat transfer problems encountered in practice are transient in nature, but they are usually analyzed under some presumed steady conditions since steady processes are easier to analyze. Transient heat transfer analysis is obviously of significant practical interest because of the large number of heating and cooling processes which must be calculated in industrial applications. The proposed extrapolation method can apply to transient heat transfer case. In this case, extrapolation can be performed along time axis. To obtain the transient temperature profile, traditional heat transfer partial differential equation is solved by MATLAB PDE Tool Box.

To obtain the temperature profile, heat transfer experiment is performed to experiment furnace model. Fig. 3.3 shows the furnace geometry. The furnace is 36 inch long and 8 inch in diameter. The heat source applies at the bottom and the hot air exhausts to the top. To obtain the temperature profile, traditional heat transfer governing partial differential equation is used.

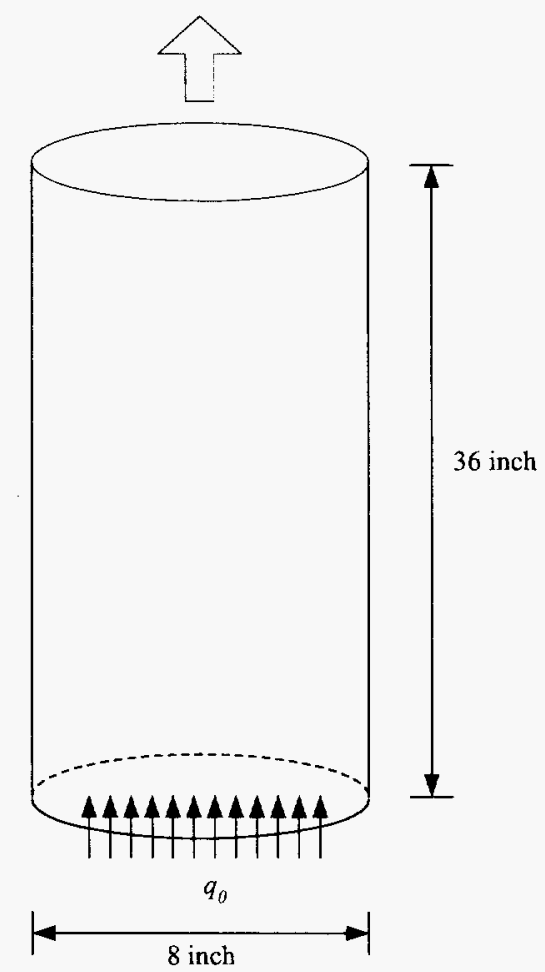

Fig. 3.3. Furnace geometry.

In this research, the general finite element method (FEM) is used to solve partial differential equation (PDE) [48]. The numerical algorithm is fully built in a PDE module of a commercial code MATLAB ${ }^{\mathrm{TM}}$. The governing equation is computed numerically on triangular unstructured grids generated by advancing front method or Delauny triangulation.

Fig. 3.4 shows the mesh of the geometry. Since the unit and problem are transformed inch to $\mathrm{cm}$ and $3 \mathrm{D}$ to $2 \mathrm{D}$ (axisymmetric), the furnace has $0.9 \mathrm{~m}$ length and $0.1 \mathrm{~m}$ in diameter. 


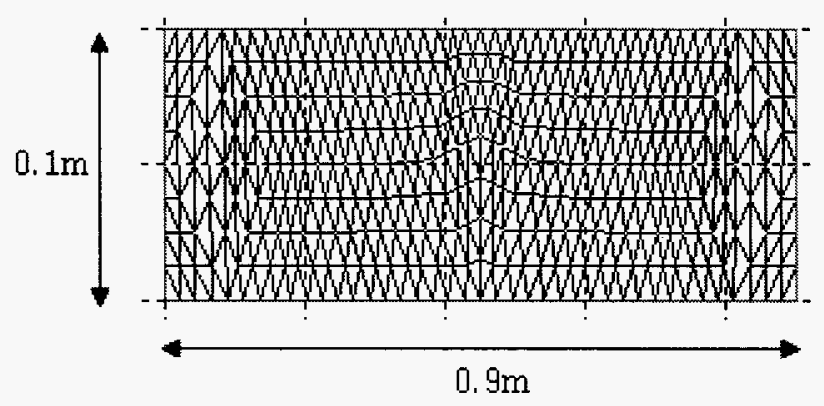

Fig. 3.4. Mesh of geometry.

\subsubsection{Governing Equation}

In all the following equations, the Laplacian is defined for cylindrical coordinates as

$$
\nabla^{2} T=\frac{1}{r} \frac{\partial}{\partial r}\left(r \frac{\partial T}{\partial r}\right)+\frac{\partial^{2} T}{\partial z^{2}}
$$

The fundamental equations of mass and heat transfer are derived from the conservation laws of mass, momentum, and energy, respectively. Since the furnace shape is cylindrical and symmetrical, the relevant equations become:

\section{Continuity:}

$$
\vec{\nabla} \cdot \vec{V}=0 \text { or } \frac{1}{r} \frac{\partial}{\partial r}\left(r u_{r}\right)+\frac{\partial u_{z}}{\partial z}=0
$$

r-momentum:

$$
\rho \frac{\partial u_{r}}{\partial t}+(\vec{V} \cdot \vec{\nabla}) u_{r}=-\frac{\partial P}{\partial r}+\mu\left\{\frac{1}{r} \frac{\partial}{\partial r}\left(r \frac{\partial u_{r}}{\partial r}\right)+\frac{\partial^{2} u_{r}}{\partial z^{2}}-\frac{u_{r}}{r^{2}}\right\}
$$

\section{z-momentum:}

$$
\rho \frac{\partial u_{z}}{\partial t}+(\vec{V} \cdot \vec{\nabla}) u_{z}=\rho g \beta\left(T-T_{\infty}\right)-\frac{\partial}{\partial z}\left(P-P_{\infty}\right)+\mu\left\{\frac{1}{r} \frac{\partial}{\partial r}\left(r \frac{\partial u_{z}}{\partial r}\right)+\frac{\partial^{2} u_{z}}{\partial z^{2}}\right\}
$$

\section{Energy:}

$$
\frac{\partial T}{\partial t}+(\vec{V} \cdot \vec{\nabla}) T=\alpha\left\{\frac{1}{r} \frac{\partial}{\partial r}\left(r \frac{\partial T}{\partial r}\right)+\frac{\partial^{2} T}{\partial z^{2}}\right\}+\frac{\dot{q}}{\rho c_{p}}+\text { Viscous }
$$


where the thermal diffusivity is defined as $\quad \alpha=\frac{k}{\rho c_{p}}$

Eqs. (3.3) and (3.4) are called Navier-Stokes equations, and the buoyancy effect is coupled in Eq. (3.4). Eq. (3.5) represents the conservation of energy, or the first law of thermodynamics. This system of PDE's is highly nonlinear and coupled with each other, so therefore the general statevariable integration approach which often works in linear systems is impossible for this case due to the heavy computational load. Instead of the full simulation, we linearize this system by using simple assumptions of approximation. For example, by assuming low viscosity, and the existence of natural convection, the following PDE describes the given system.

$$
\frac{\partial T}{\partial t}=\alpha_{e f f} \nabla^{2} T
$$

Fig. 3.5 shows the boundary conditions that will be applied and which are now described.

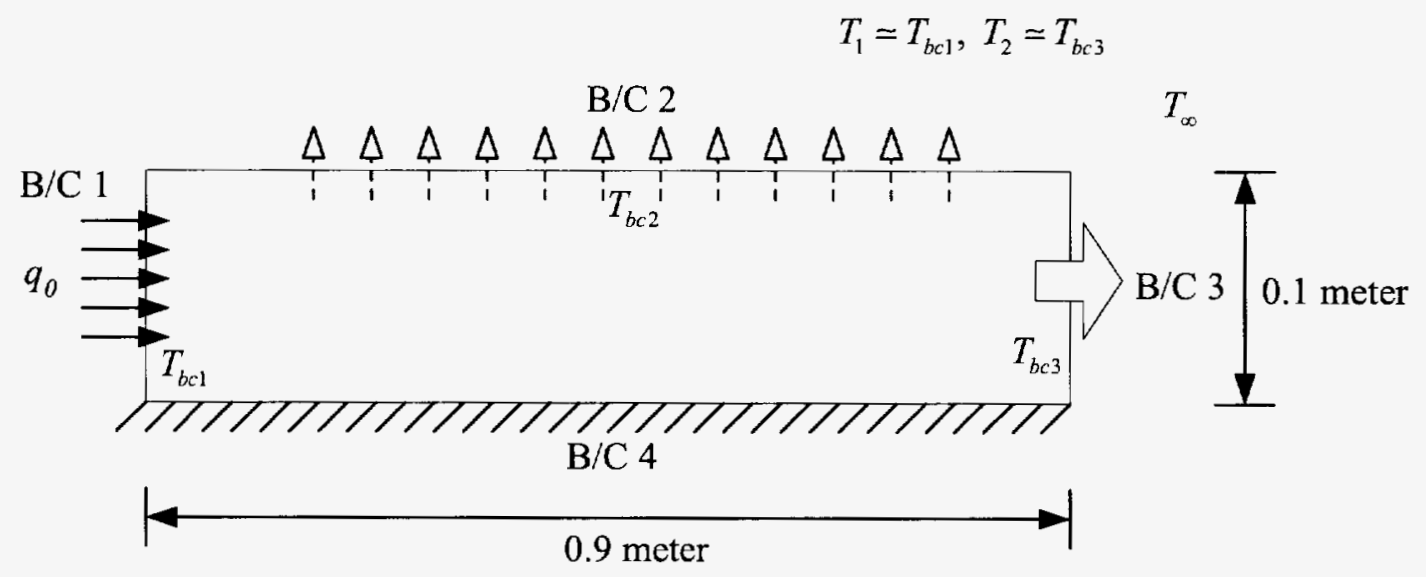

Fig. 3.5. Boundary conditions.

\section{B/C 1: heat inlet boundary condition:}

Let the cylinder have a diameter, $D$, and a length, $L$. The inlet heat flux is assumed to be a singular distribution at the central axis. The heat is concentrated at the center because the burner is located there.

$$
\dot{q} / A=-\frac{q_{0}}{r}=-\left.k_{e f f} \frac{\partial T}{\partial z}\right|_{z=0} \quad \text { for } \mathrm{B} / \mathrm{C} 1
$$

The constant $q_{0}$ is generally unknown, so we will find a suitable value in the numerical procedure. 


\section{B/C 2: convection boundary condition (1):}

The outgoing heat flux is given by the following equation,

$$
\dot{q} / A=h\left(T-T_{\infty}\right)=-\left.k_{e f f} \frac{\partial T}{\partial r}\right|_{r=D / 2} \quad \text { for } \mathrm{B} / \mathrm{C} 2
$$

\section{B/C 3: convection boundary condition (2):}

The outlet heat flux is given by the following equation,

$$
\dot{q} / A=h\left(T-T_{\infty}\right)=-\left.k_{e f f} \frac{\partial T}{\partial z}\right|_{z=L} \quad \text { for } \mathrm{B} / \mathrm{C} 3
$$

\section{B/C 4: symmetric boundary condition :}

Assuming the entire bottom edge is insulated, which produces an adiabatic condition, the boundary condition becomes

$$
\frac{\partial T}{\partial r}=0 \quad \text { for } \mathrm{B} / \mathrm{C} 4
$$

along the bottom edge.

\subsubsection{Numerical Procedure}

After several experiments, the final value is $q_{0}=250[\mathrm{~W} / \mathrm{m}]$ for this case where the atmospheric condition is fixed to $T_{\infty}=20^{\circ} \mathrm{C}=293 \mathrm{~K}$. Each transient solution is stored at a given time, and the field data can be presented in the matrix form from some manual data manipulation of MATLAB $^{\mathrm{TM}}$. Fig. 3. 6 shows the entire procedures of obtaining temperature profiles. 


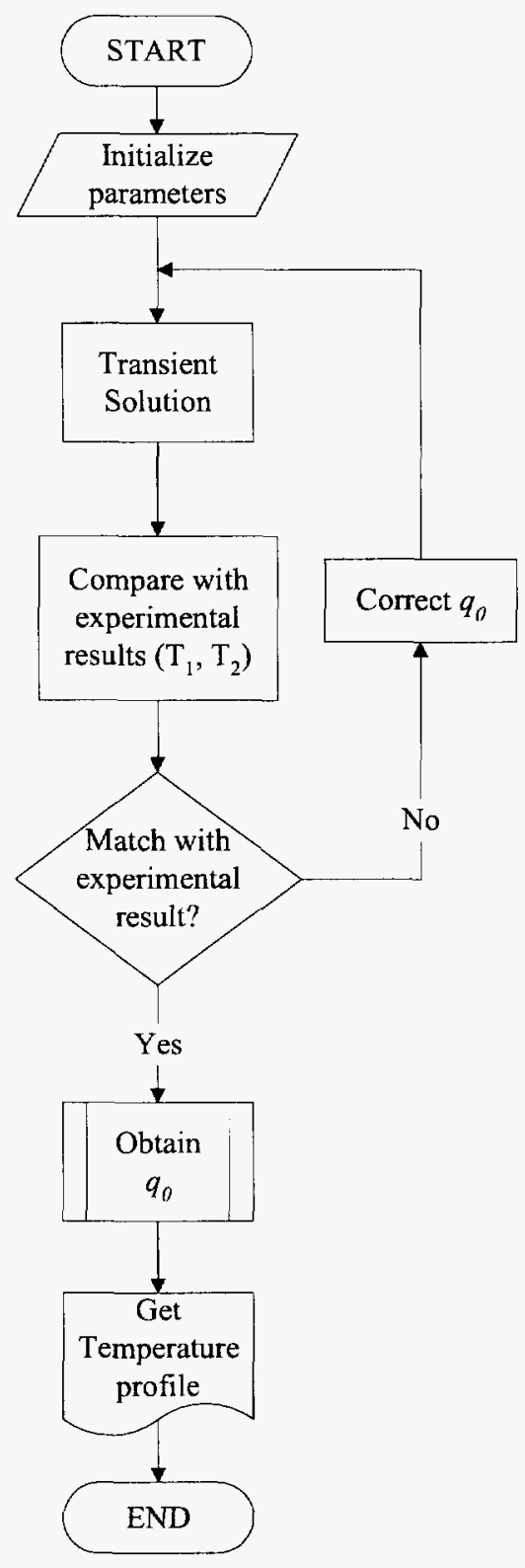

Fig. 3.6. Flow chart for overall procedure.

\subsubsection{Failures/shortcomings of Conventional ANN's}

Recently, a shift has occurred in the overall architecture of neural networks from simple or component-type networks to system-type architectures. The most popular architecture seems to be the one advocated by Jacobs and Jordan [31], called the "Modular Connectionist Architecture", one example of which is shown in Fig. 3.7 [32]. It consists of a collection of expert components, each being trained independently, tied together by a component called the "gating logic" element, whose function is to decide on the relative contributions to be made by 
each expert component, such that when they are added, they provide the correct output for a given input. The present proposed method represents an adaptation of Fig. 3. 7.

The most serious flaw in the design of system-type neural networks is the lack of a cohesive discipline in the architectural design and in the design of the learning algorithm. Virtually, the entire design is done on an intuitive basis. As a contrast to intuition, the proposed method relies on semigroup theory for the design of the semigroup channel.

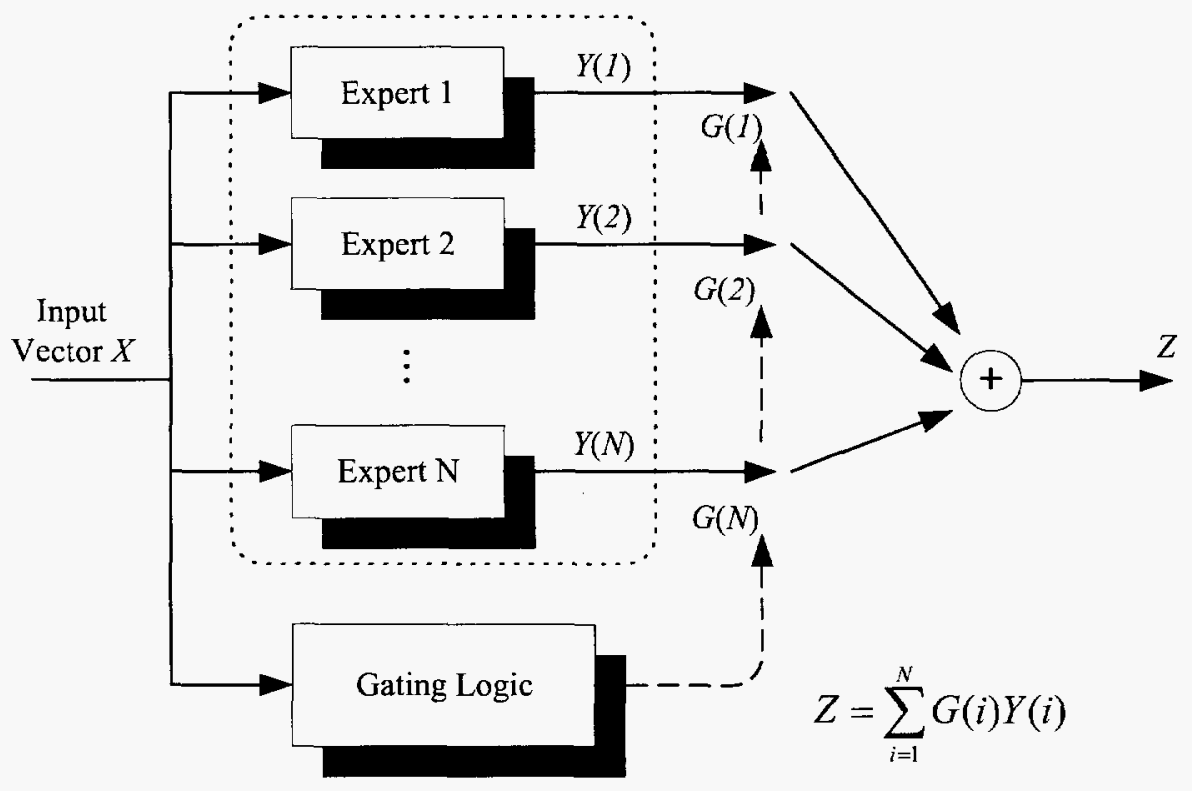

Fig. 3.7 Modular connectionist architecture.

To illustrate the lack of a cohesive discipline, in [32], the partitioning of components corresponds to separation of variables, which works if the variables are separated and does not work if the variables are not separated.

\subsubsection{Semigroup Theory}

In recent years, among many other applications, semigroup theory has been widely used in the study of control and stability of systems governed by differential equations on an abstract Banach space. It is well known that differential equations form a major tool in the study of pure and applied sciences including engineering and many areas of social sciences. Depending on the problem, these equations may take various forms, such as functional differential equations, partial differential equations (PDE's), and sometimes combination of interacting systems of ordinary and partial differential equations. In general, under broad assumptions, many of these equations can be reformulated as ordinary differential equations on abstract spaces, for example, Banach spaces. Once this is done, various "denseness" theorems then provide the basis for forming a finite-dimensional approximation. This is where semigroup theory plays an important role and provides a unified and powerful tool for the study of existence, uniqueness, and continuous dependence of solutions on parameters and their regularity properties. Even though the field appears to be highly developed, there is no lack of interesting problems. In fact, there 
are formidable challenges of purely mathematical nature, not encountered in pre control era, which must be resolved to solve some of the notoriously difficult problems presented by the needs of current technology. Semigroup theory has also found extensive applications in the study of Markov process, ergodic theory, approximation theory and control and stability theory [33].

\section{Definition}

Let $X$ be a Banach space and $\{T(t), t \geq 0\}$ a family of bounded linear operators in $X$, such that for each $t \geq 0, T(t) \in \mathscr{L}(X)$ where $\mathscr{L}(X)$ denotes the space of bounded linear operators in $X$. The family of operators $\{T(t), t \geq 0\}$ is said to be a semigroup of operators in $X$ if

(i) $T(0)=\mathrm{I} \quad$ (Identity)

(ii) $T(t+s)=T(t) T(s)=T(s) T(t) \quad$ for all $t, s \geq 0$.

(iii) $T$ is continuous for $t \geq 0$.

To give an idea of a semigroup property being possessed by a mapping, consider the following steady state heat flow model in Cartesian coordinates:

$$
\frac{\partial^{2} T}{\partial x^{2}}+\frac{\partial^{2} T}{\partial y^{2}}=0
$$

If we set $T(x, y)=C(y)^{T} E(x)=c_{1}(y) e_{1}(x)+c_{2}(y) e_{2}(x)$, where $e_{i}$ are orthonormal basis, then by substitution, $c_{1}^{\prime \prime} e_{1}+c_{2}^{*} e_{2}=-c_{1} e_{1}^{*}-c_{2} e_{2}^{*}$. This, in turn implies

$$
\begin{aligned}
& c_{1}^{\prime \prime}+c_{2}^{\prime \prime}<e_{2}, e_{1}>=-c_{1}<e_{1}^{\prime \prime}, e_{1}>-c_{2}<e_{2}^{\prime \prime}, e_{1}> \\
& c_{1}^{\prime \prime}<e_{1}, e_{2}>+c_{2}^{\prime \prime}=-c_{1}<e_{1}^{\prime \prime}, e_{2}>-c_{2}<e_{2}^{\prime \prime}, e_{2}>
\end{aligned} \text {, or }\left[\begin{array}{l}
c_{1}^{\prime \prime} \\
c_{2}^{\prime \prime}
\end{array}\right]=\mathrm{A}\left[\begin{array}{l}
c_{1} \\
c_{2}
\end{array}\right]
$$

for a suitable matrix $A$, which leads to a semigroup for $C(y)$. Notice that the semigroup property is not reflected in the original data; it appears only in the coefficient vector portion of the system description.

\subsubsection{Relationship of Semigroup Theory to Neural Network Design}

The semigroup approach [50] begins by asserting that certain functions are to be re-interpreted as follows: under certain circumstances, the function $T(r, z)$ should be thought of not as one map, but rather, as one family of maps: $\left\{T_{z}(r), z \in[0, L]\right\}$ which, in turn, is produced by a second family of maps $\{\Phi(z)\}$ where the two families are related by the following: $T_{z}(r)=C(z)^{T} E(r)$; where : $C(z)=\Phi(z) C(0)$, and where $\Phi\left(z_{1}+z_{2}\right)=\Phi\left(z_{1}\right) \Phi\left(z_{2}\right)$.

This interpretation suggests that the mapping must be achieved with a pair of neural networks, one that selects a given function at each value of $z$ and another that then implements the chosen 
function. This interpretation also places severe constraints on the "selecting" neural component, forcing it to take on the generic semigroup behavior: $\Phi\left(z_{1}+z_{2}\right)=\Phi\left(z_{1}\right) \Phi\left(z_{2}\right)$.

If a given system behavior (expressed as a set of data) possesses a semigroup property, the extrapolation of that data set is achieved by a neural network (the semigroup channel) which itself acquires its own semigroup property. The semigroup property is ultimately achieved within the semigroup channel as a sequence of weight changes that occur after weight convergence has taken place.

\subsubsection{Proposed Neural Network Architecture.}

Neural networks are being used for systems described by PDE's [35]. The system-type attribute of the neural network architecture is shown in Fig. 3. 8, implementing an arbitrary function $T(z, r)$. Unlike conventional neural network architectures that would attempt to achieve the mapping $T(z, r)$ with one neural network, the proposed architecture reflects a system-type approach using two neural network channels, a Function Channel and a Semigroup Channel, in an adaptation of the connectionist architecture (Fig. 3.7). During use, the semigroup channel supplies the function channel with a coefficient vector $C(z)$ as a function of the index $z$. The coefficient vector, when applied to the basis set $E(r)$ of the function channel, causes the function channel to operate as one specific function from within a vector space of functions. Jointly, these two channels realize a semigroup-based implementation of the mapping $T(z, r)$. The similarity between the proposed architecture (Fig. 3.8) and that of Fig. 3.7 arises from the fact that the Function channel is implemented as $\mathrm{N}$ "expert" systems.

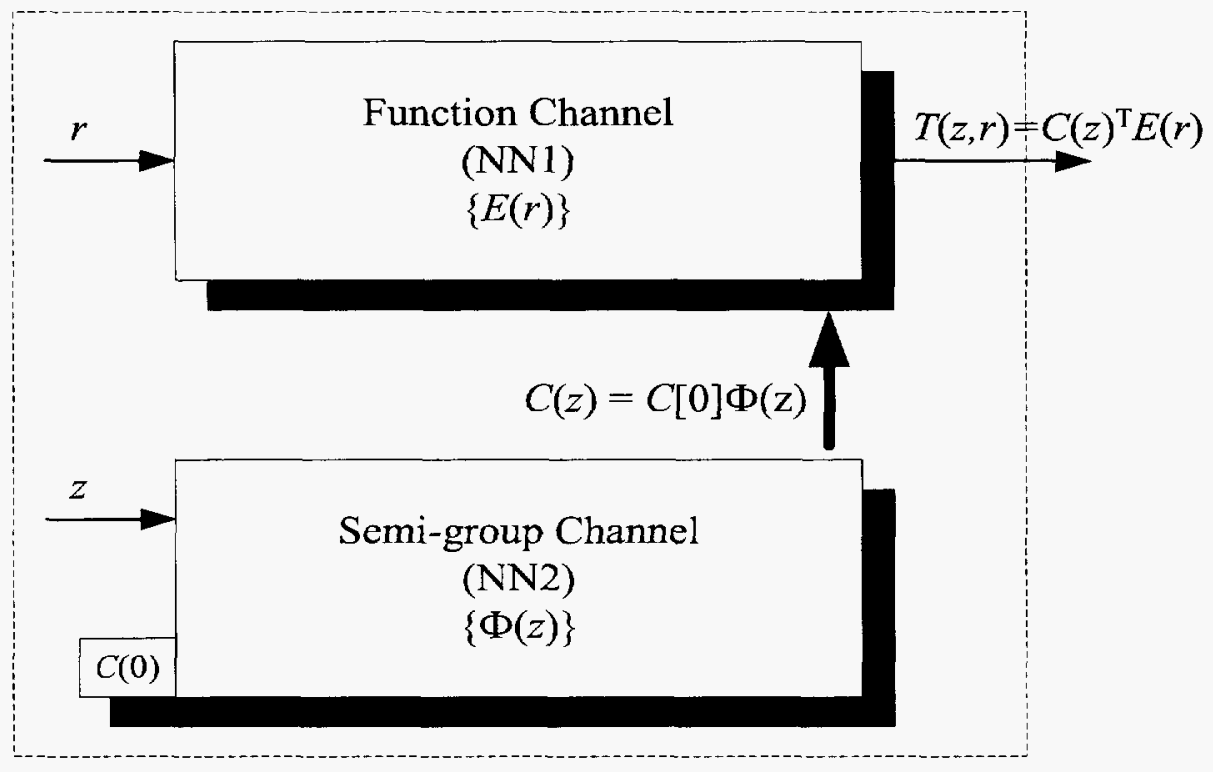

Fig. 3.8. System-type architecture.

The function channel can have a Radial Basis Function (RBF) architecture [36]. It consists of $n$ RBF networks, each one of which implements one orthonormal vector of an $n$-dimensional basis 
set of vectors $E(r)$. The outputs of the orthonormal vectors are (internally) linearly summed so that the channel spans an $n$-dimensional function space. The coefficients which determine the linear sum and thereby define the specific function being implemented is supplied by the semigroup channel. Up to this point, the operation of the RBF channel parallels the idea used by Phan and Frueh [37]. One of the essential differences between their approach and the present proposed approach is that the former requires prior engineering knowledge for selecting the basis vectors, and the latter approach requires no such knowledge. One advantage that RBF networks have over other architectures is that their functionality can be given an explicit mathematical expression in which the neuron activation functions act as Green's functions [38], [39]. This makes these networks amenable to design rather than training. Another advantage is that they function as universal approximators [36]. The semigroup channel can be adapted from the Diagonal Neural Network (DRNN) [40], [41] or the Simple Recurrent Network (SRN) architecture [42], in which the input is split into a dynamic scalar component $z$ and one static vector component, the vector $C(0)$. The output is a vector $C(z)$, which is related to the dynamic input $z$ and to the static input $C(0)$ by the semigroup property: $C(z)=\Phi(z) C(0)$, where $\Phi\left(z_{1}+z_{2}\right)=\Phi\left(z_{1}\right) \Phi\left(z_{2}\right)$. (Refer to Fig. 3. 8).

\subsubsection{Learning Algorithm of Proposed System-type NN}

The first component of the system, namely the Function Channel, since it is composed of RBF components, can be designed, rather than trained. The second component, the Semigroup Channel, can be trained in the new way illustrated below. During training, the semigroup channel receives as input a preliminary coefficient vector $C(z)$ and produces a smoothened coefficient vector $\tilde{C}(z)$. That is, the primary objective of training is to replicate (and, if necessary, to smoothen) the vector $C(z)$ with a vector $\tilde{C}(z)$ which has the following semigroup property [43]: $\tilde{C}(z)=\Phi(z) \tilde{C}(0)$, where $\tilde{C}(\mathrm{z}) \equiv\left[\tilde{\mathrm{c}}_{1}(z), \tilde{\mathrm{c}}_{2}(z), \ldots, \tilde{\mathrm{c}}_{\mathrm{n}}(z)\right]^{T}$ and $\Phi(z)$ is an $n \mathrm{x} n$ matrix that satisfies: $\Phi\left(z_{1}+z_{2}\right)=\Phi\left(z_{1}\right) \Phi\left(z_{2}\right)$. However, there is a secondary objective of training; the channel must also "replicate" the semigroup property of the trajectory by gradually acquiring a semigroup property of its own, in weight space. The existence of this acquired semigroup property in weight space becomes the basis for extrapolation. In order to elicit this gradual acquisition of the semigroup property, it is necessary that the training in this second step (semigroup tracking) occur in a gradual manner, as shown in Fig. 3.9. While the modified SRN is being trained, there are two kinds of convergence involved. First, at each training step, the weights must converge. At this point the neural network is able to duplicate the trajectory up to that point. This training (along with the accompanying convergence) must re-occur for the next step. This training/convergence re-occurs for each subsequence step. At some future point, in addition to the convergence at each step, there must be a convergence in the overall pattern of weights. This latter convergence will be referred to as the "second weight convergence". In old fashion, the entire trajectory is used as the learning target; in Fig. 3.9, the entire trajectory is split into successively-longer sub-trajectories. 


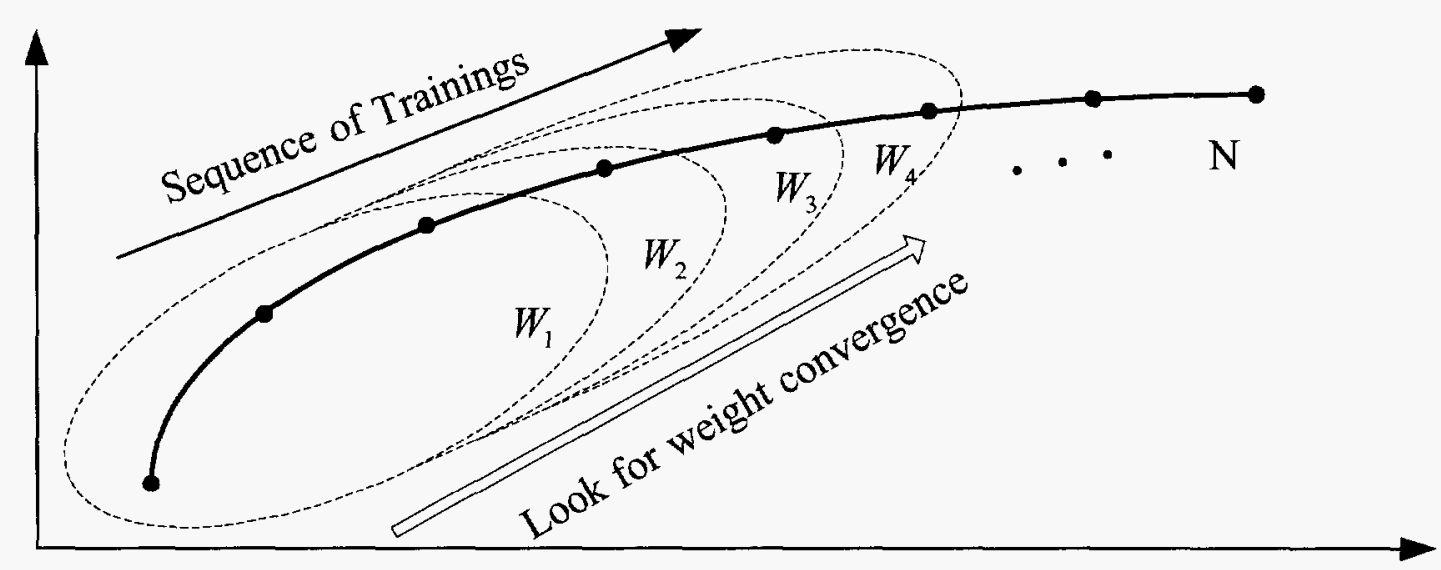

Fig. 3.9. Overview of new training algorithm.

It must be noted that there are two concepts of convergence that occur. First, to acquire a given weight, for example, weight $W_{3}$, requires conventional training convergence, which in turn may require 500 training iterations. Second, after all weights $\left(W_{1}, W_{2}, \ldots, W_{n}\right)$ have been obtained, a search begins for a convergence within this weight stream alone. Again, unlike conventional neural network training which (using either a batch method or an incremental method) would repeatedly present the entire trajectory as a target, the proposed method begins by slicing the entire trajectory into a nested sequence of trajectories. In the nested sequence a given trajectory includes the previous trajectory and adds one additional data point. Each sub-trajectory is trained by a conventional (batch) method, and the resulting weight is recorded. After all sub-trajectories have been trained (culminating in the last sub-trajectory being the entire trajectory), the sequence of resulting weights is examined for convergence. Only if this second convergence is achieved, can extrapolation begin.

Extrapolation involves only the coefficient vector and the SRN network (the semigroup channel). At the uppermost level, the idea is to train the neural network to replicate the coefficient vector (produced by the previous system modeling effort) in such a way that it is additionally replicating the semigroup property, which is responsible for generating the coefficient vector by acquiring a semigroup property of its own in weight space. As a comparison, some other recent extrapolation attempts are given in [44]. One current method, which also attempts to build a universal framework for extrapolation, occurs in various forms in nonlinear control theory and is collectively called "continuation methods." These methods have been in existence for some time, but are only recently receiving attention [45].

\subsubsection{System Modeling}

The modeling and extrapolation problem is formulated as follows. Given a set of empirical data for which there is no analytic description, first develop an analytic model for the data, and then extrapolate the model along one specific axis. System modeling is achieved through a technique referred to as algebraic decomposition. Algebraic decomposition is an operation which is applied to a given function $T(r, z)$, for the purpose of representing it in a form that contains a semigroup: 
$T(r, z)=T_{z}(r)=C(z)^{T} E(r)$, where $E(r)$ provides the algebraic basis for the representation of each member of the parameterized function $\left\{T_{z}(r)\right\}$. The essential value of algebraic decomposition is that when it is applied to the class of functions that will be considered in this proposed research, it always produces a semigroup property for the coefficient vector.

\subsubsection{Extrapolation}

Extrapolation involves only the coefficient vector and the SRN (the Semigroup Channel). At the uppermost level, the idea is to train the neural network to replicate the coefficient vector (produced by the previous system modeling effort) in such a way that it is additionally replicating the semigroup property, which is responsible for generating the coefficient vector by acquiring a semigroup property of its own in weight space. This idea requires a gradual training approach in which the sequence of weight changes reaches a point of weight convergence, after which only that portion of the weight changes that are connected to the extrapolating variable experience any subsequent changes. When the weights converge, SRN can be considered as a linear system, in which the rule for weight change (for the extrapolating variable) generates a semigroup property.

\subsection{Simulation and Estimation Results}

\subsubsection{Monitoring of Temperature Distribution in Boiler Furnace}

The following illustrates simulation results of the application of the proposed method to the prediction (extrapolation) of temperature data from a boiler furnace of dimensions comparable to that found in a power plant. The data represents "raw data" furnished by the Penn State Energy Institute. The geometry of the furnace is cylindrical with the $z$-axis along the furnace axis, and with $r$ going from one wall to the other wall. (Note that $r$ is a diameter, not a radius.) A simulation will be performed on the configuration below, where there are 25 probes, each one providing 11 readings. The extrapolation will be simulated in the region occupied by probes 25 to 30 , Fig. 3. 10. The results of the extrapolation will be compared to given raw data in that region. The temperature distribution is shown in Fig. 3.11.

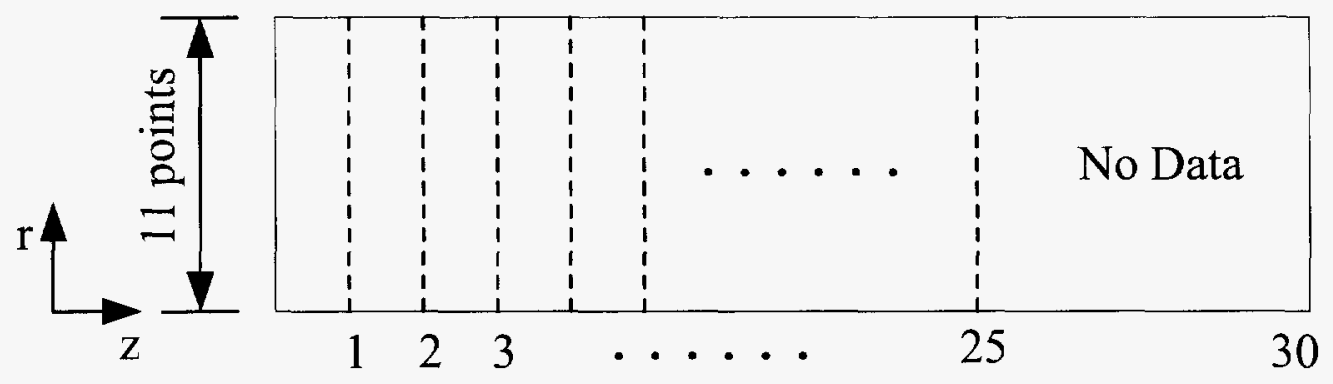

Fig. 3.10. Temperature probe configuration for the furnace. 


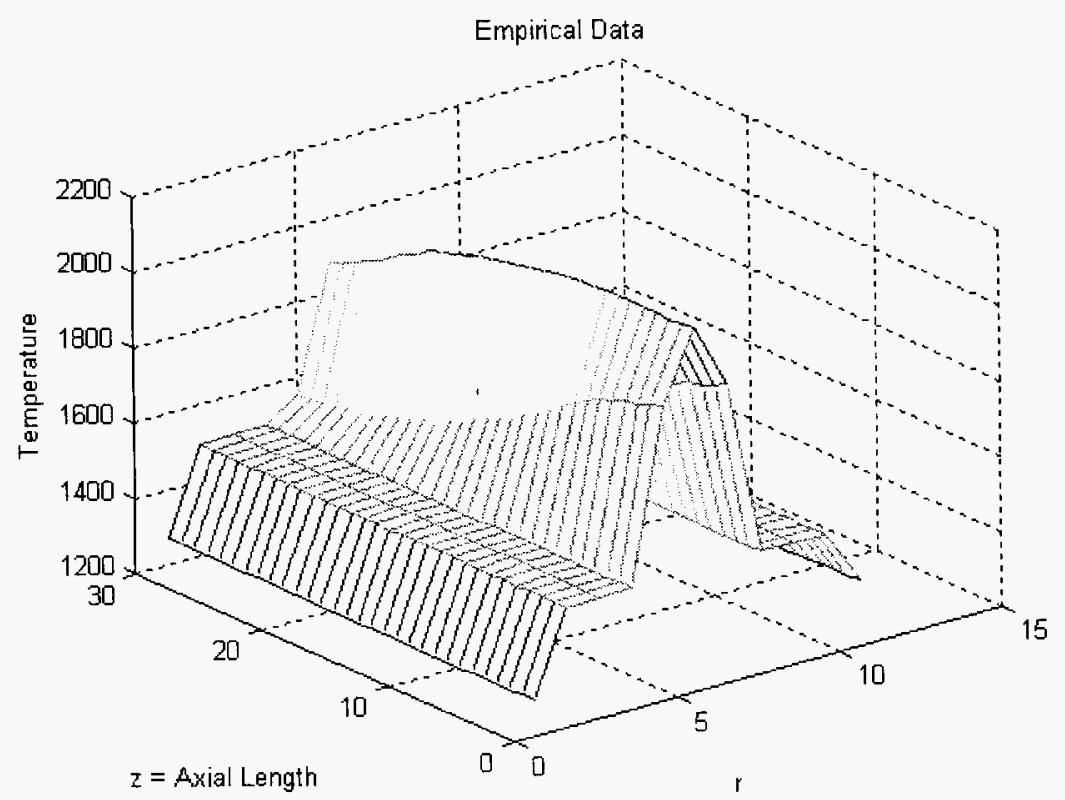

Fig. 3.11. Temperature distribution for the furnace.

The preliminary (rough) coefficient vectors produced by the RBF network are shown in Fig. 3.12. The use of this rough coefficient vector together with the basis set of vectors can produce the computed temperature distribution shown in Fig. 3.13.

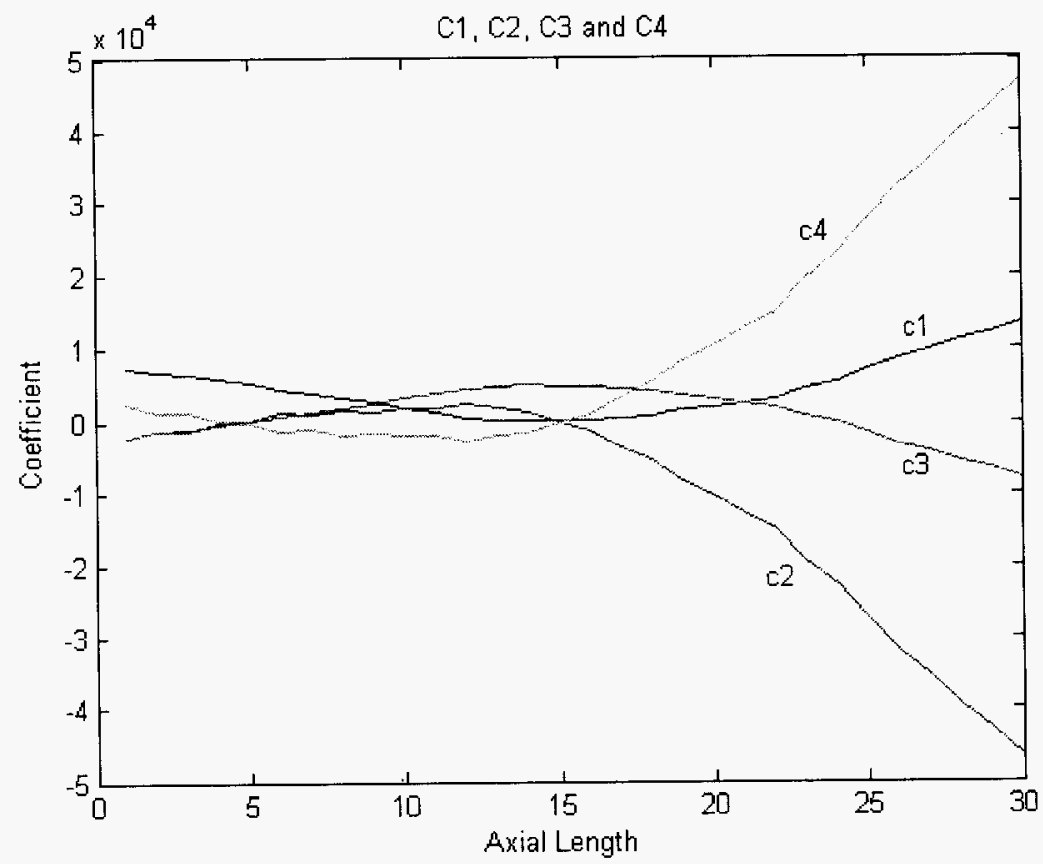

Fig. 3.12. Preliminary coefficient vector set. 


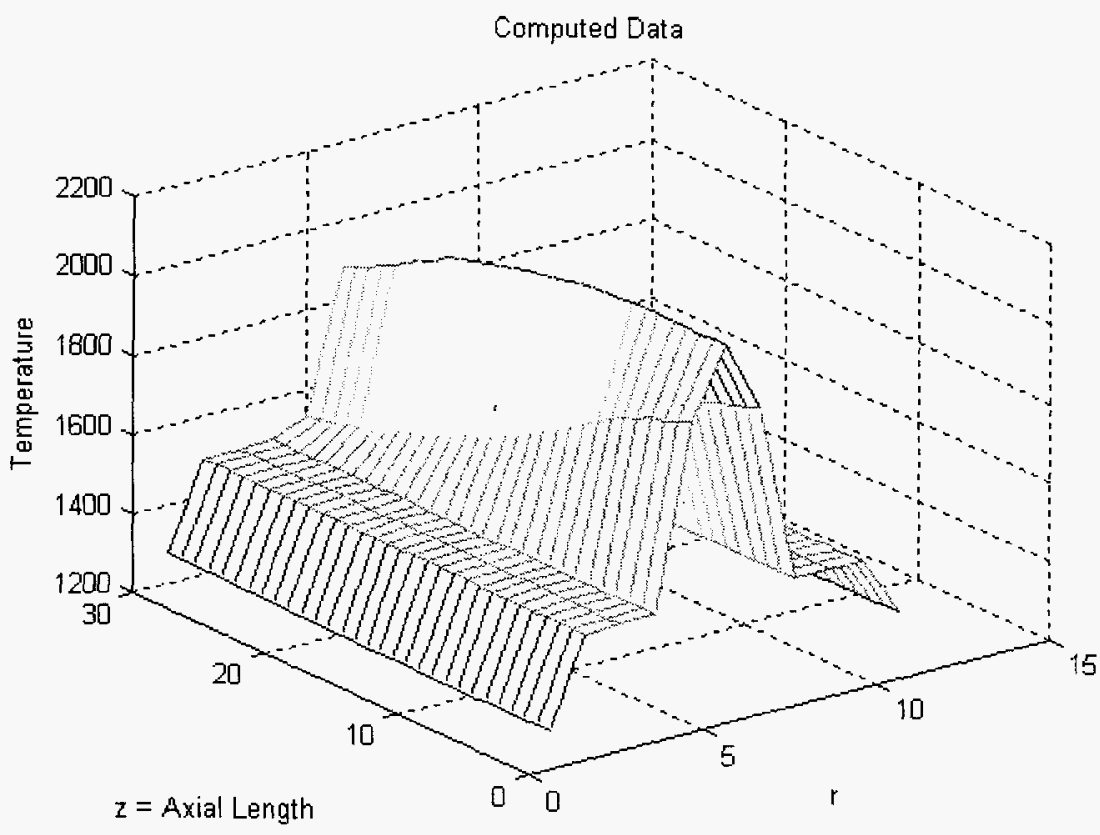

Fig. 3.13. Computed temperature.

The possibility for extrapolation begins by checking for weight convergence as training is performed along the coefficient vector. In this case, weight convergence occurs as this training is repeated over successively longer intervals (refer Fig. 3.9). It is this weight convergence, which becomes the basis for extrapolation. In this case, because of the smoothness, the possibility for extrapolation exists and the next step is to apply an extrapolation test in which the trailing end of the weight change sequence (produced by training) is replaced by an equivalent weight change sequence based on a rule that generates a semigroup. Based upon an observation of the weight change sequence on the interval from 15 to 20 , a semigroup-based rule for weight change is formulated and applied to the interval from 20 to 25 , as a test. Extrapolation (to the region where no data were assumed) consists of the autonomous continuation of the rule for weight change, which was derived during the extrapolation test. These results are shown in Fig. 3.14 below (only the first two coefficients are shown). 


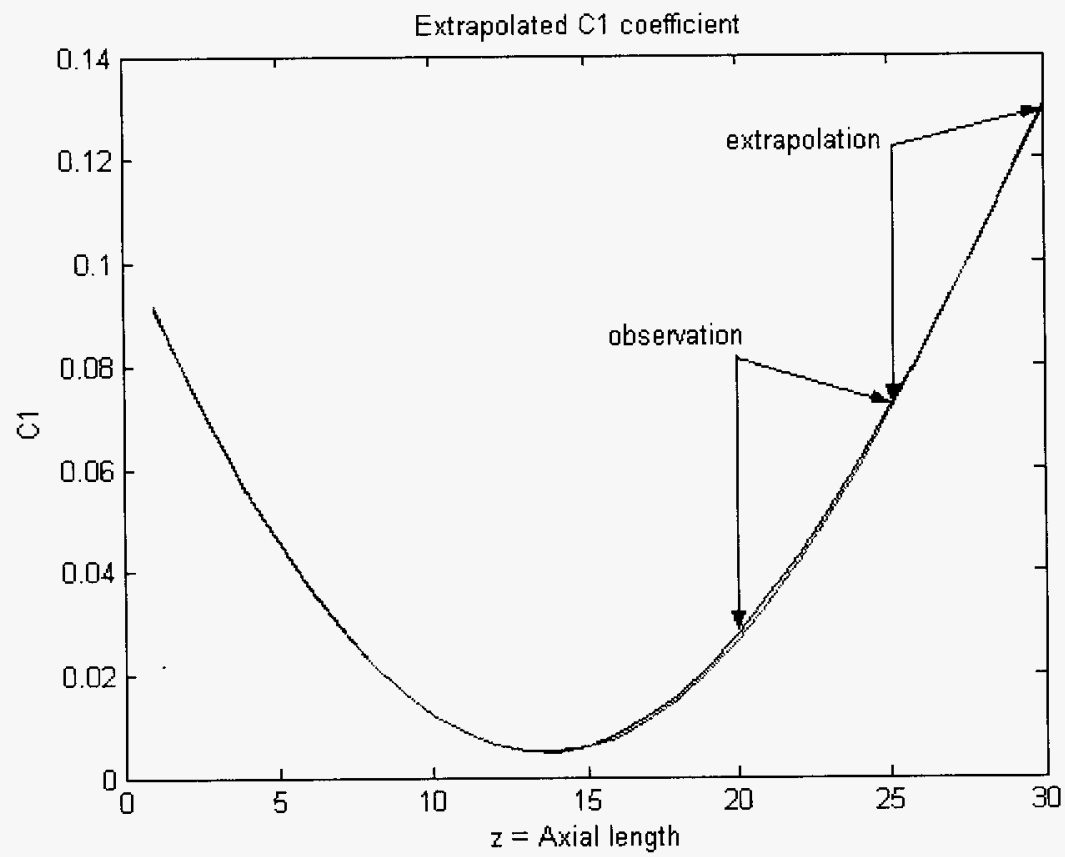

Fig. 3.14a. Extrapolation results for $\mathrm{C} 1$.

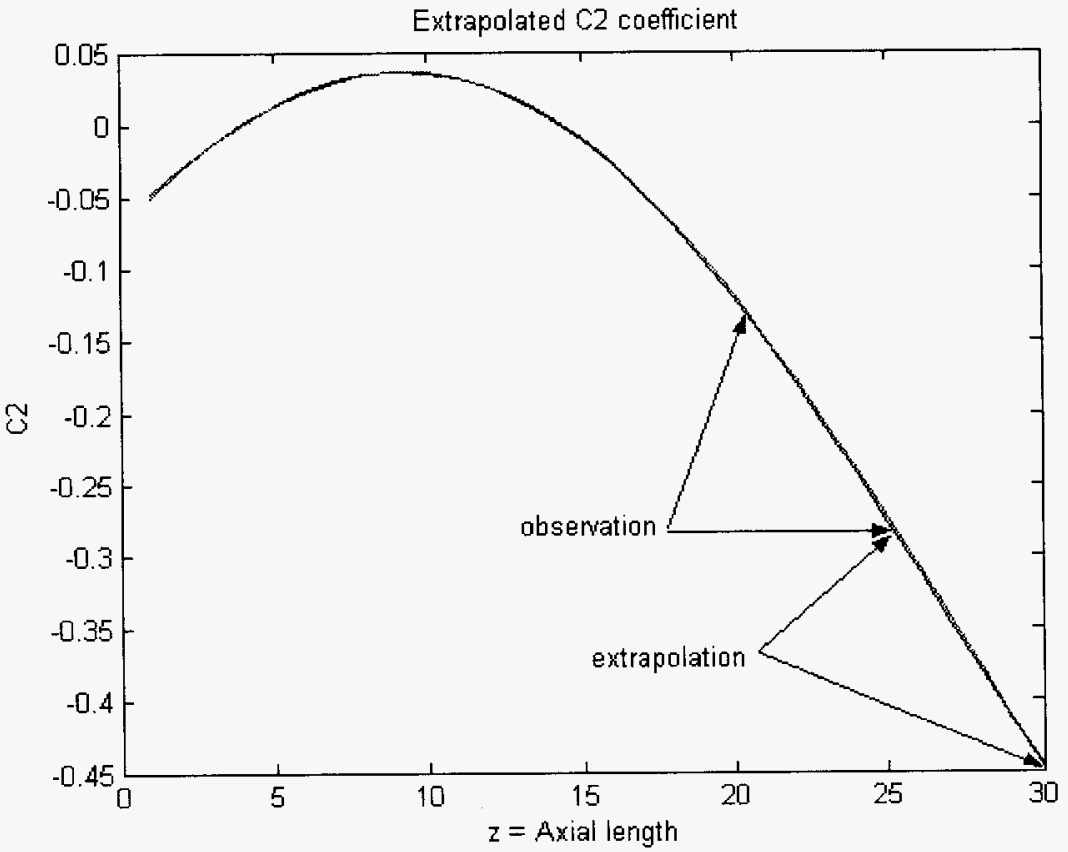

Fig. 3.14b. Extrapolation results for C2. 


\subsubsection{Demonstration of Extrapolation Capability for Enthalpy in a Power Plant}

\subsubsection{Water Enthalpy}

The water enthalpy data corresponding to the temperature and pressure is obtained from the NIST Chemistry WebBook [46]. The range of temperature is $100^{\circ} \mathrm{F}$ to $400^{\circ} \mathrm{F}$ and the range of pressure is 300 psi to 2300 psi. Although the preferred method of extrapolation in this case would be along the temperature axis, it is now demonstrated that the proposed method can be applied to the pressure-extrapolation of the enthalpy of water for temperature-pressure distributions which typically exist in the power plant at the exit of the boiler (wet stream - point 1 in Fig. 3.1). The water enthalpy is first re-expressed as the vector product: $h(T, P)=C(P) E(T)$ and extrapolation is performed along the pressure axis. Fig. 3.15 displays the error between the given empirical enthalpy and the computed enthalpy; Fig. 3. 16 displays the smoothened coefficient vector and Fig. 3. 17 displays the extrapolated coefficient vector. Based upon an observation of the weight change sequence on the interval from 1500 psi to $1750 \mathrm{psi}$, a semigroup-based rule for weight change is formulated and applied to the interval from $1750 \mathrm{psi}$ to $2000 \mathrm{psi}$, as a test. Extrapolation consists of the autonomous continuation of the rule for weight change, which was derived during the extrapolation test. Extrapolation is performed from $2000 \mathrm{psi}$ to $2300 \mathrm{psi}$ where no data were assumed based on the observation which is previously test region. 


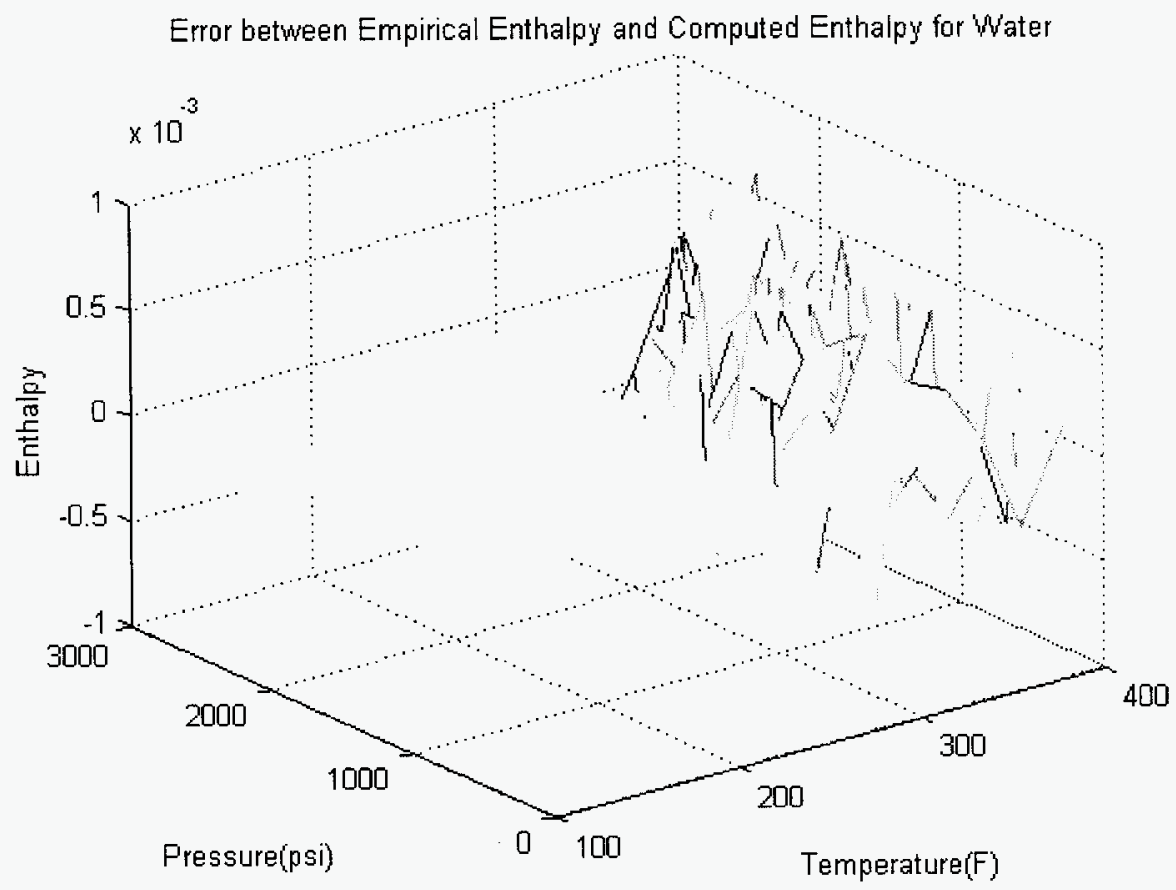

Fig. 3.15. Error between empirical and computed enthalpies.

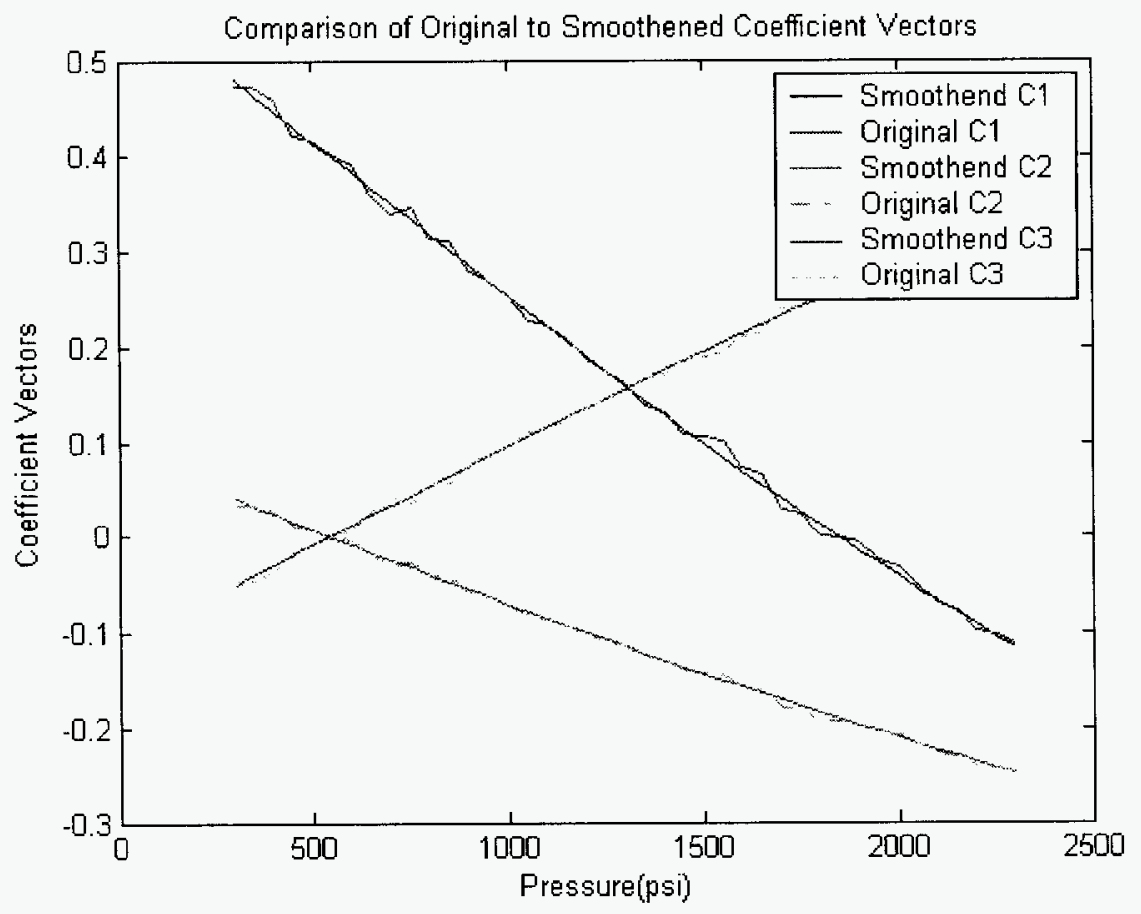

Fig. 3.16. Smoothened coefficient vectors. 


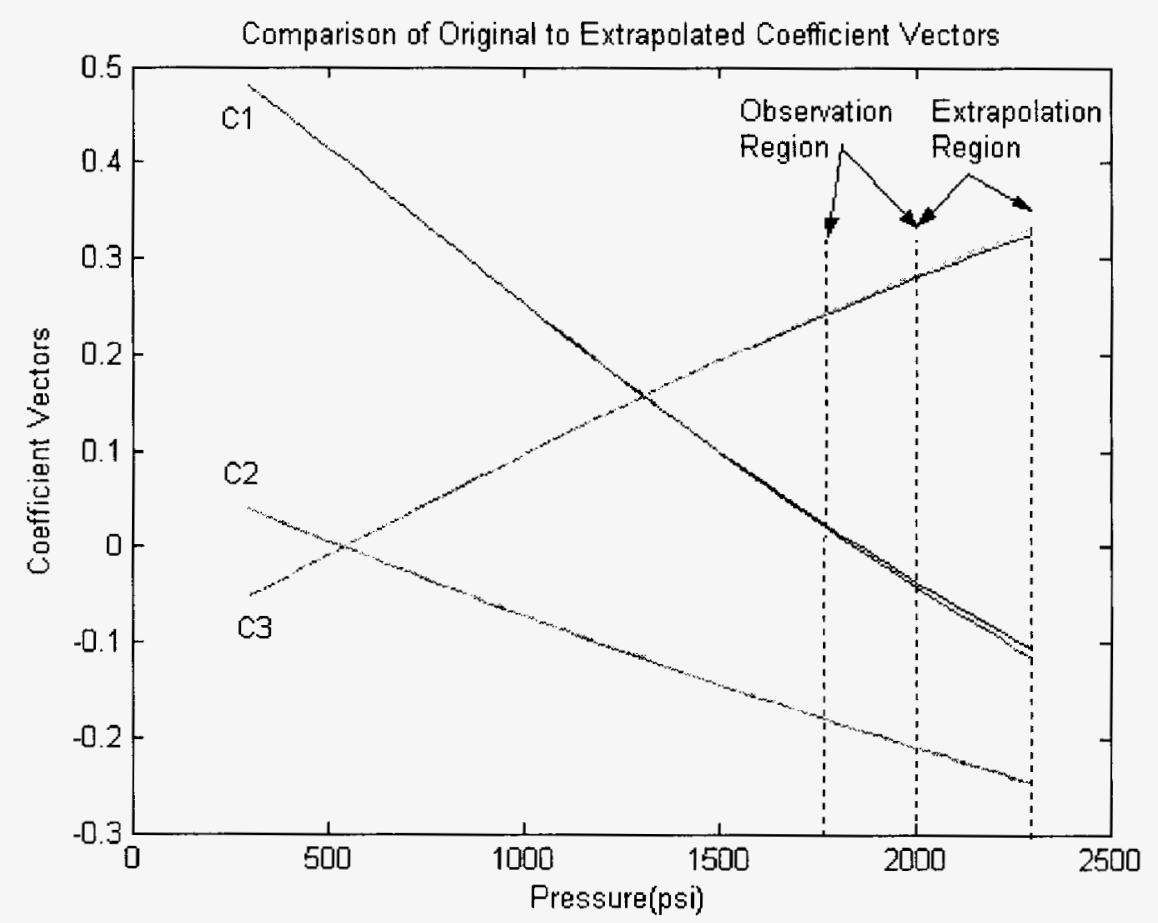

Fig. 3.17. Extrapolated coefficient vectors.

\subsubsection{Steam Enthalpy}

The steam enthalpy corresponding to the temperature and pressure is obtained from the NIST Chemistry WebBook [46]. The range of temperature is $800^{\circ} \mathrm{F}$ to $1200^{\circ} \mathrm{F}$ and the range of pressure is $800 \mathrm{psi}$ to $1500 \mathrm{psi}$. The proposed method will now be applied to the extrapolation of the enthalpy of steam for temperature-pressure distributions which typically exist in the power plant after the water has exited the boiler and the superheater and travels through the delivery section to the turbine (dry stream - points 2 to 3 in Fig. 3. 1). The steam enthalpy is first reexpressed as the vector product: $h(T, P)=C(P) E(T)$ and extrapolation is performed along the temperature axis. (Among other things, this also demonstrates that, in certain cases, extrapolation can be performed in more than one way.) Fig. 3.18 displays the error between the given empirical enthalpy and the computed enthalpy; Fig. 3.19 displays the smoothened coefficient vector and Fig. 3.20 displays the extrapolated coefficient vector. Based upon an observation of the weight change sequence on the interval from $1040^{\circ} \mathrm{F}$ to $1090^{\circ} \mathrm{F}$, a semigroup-based rule for weight change is formulated and applied to the interval from $1090^{\circ} \mathrm{F}$ to $1140^{\circ} \mathrm{F}$, as a test. Extrapolation consists of the autonomous continuation of the rule for weight change, which was derived during the extrapolation test. Extrapolation is performed from $1140^{\circ} \mathrm{F}$ to $1200^{\circ} \mathrm{F}$ where no data were assumed based on the observation which is previously test region. 


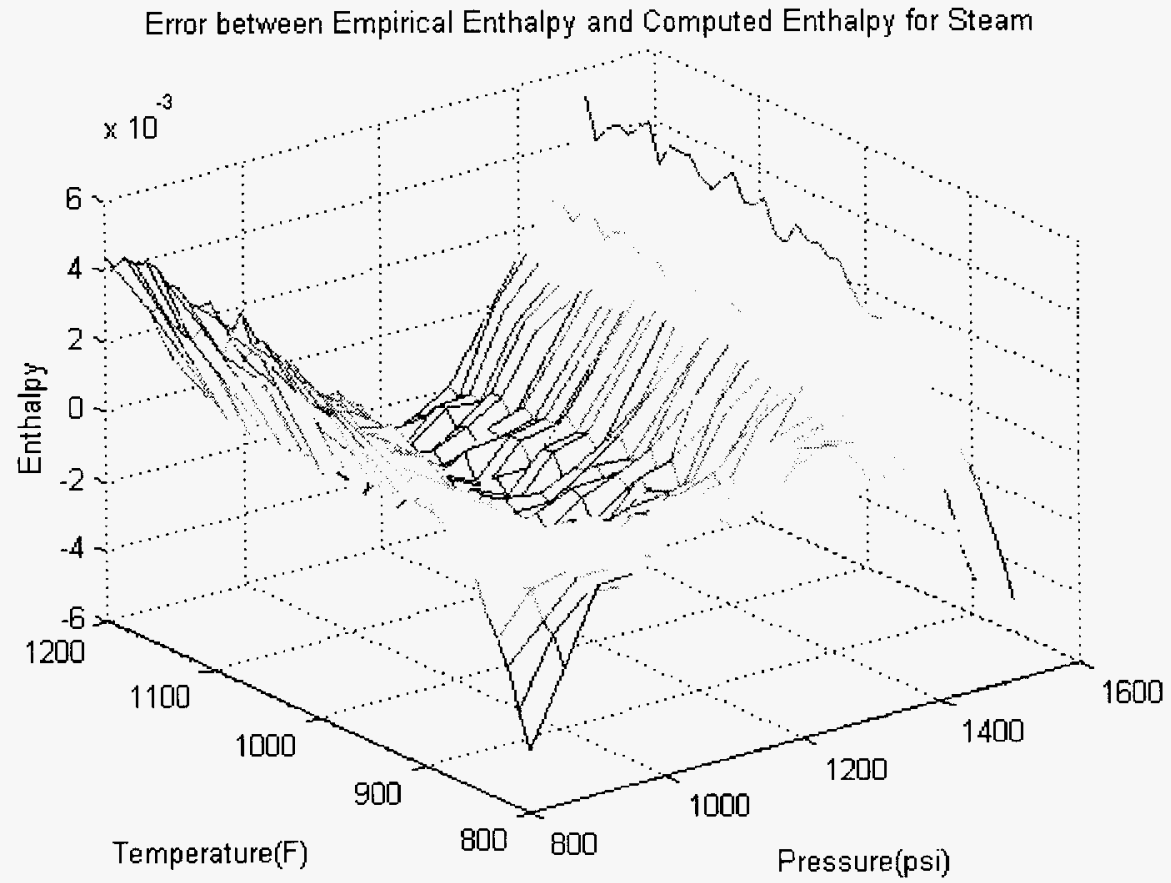

Fig. 3.18. Error between empirical and computed enthalpies.

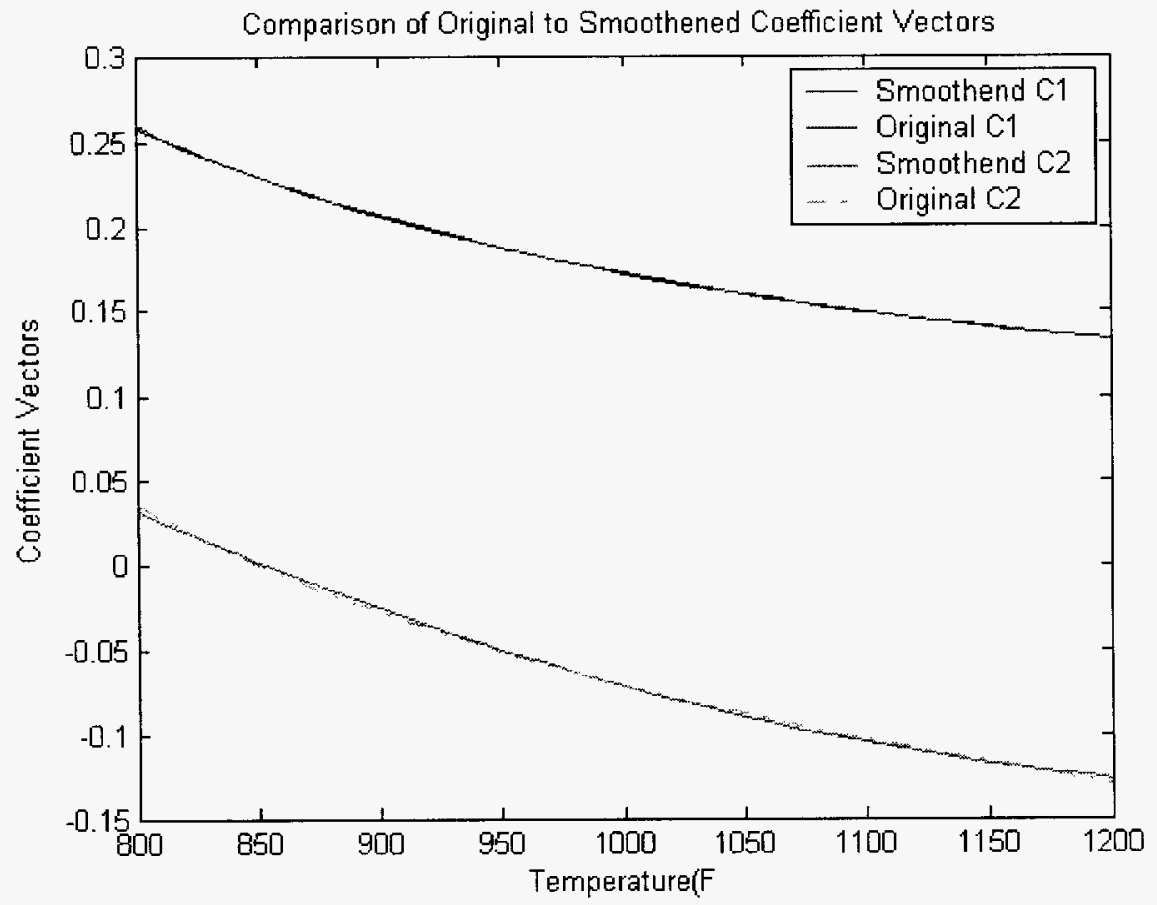

Fig. 3.19. Smoothened coefficient vectors. 


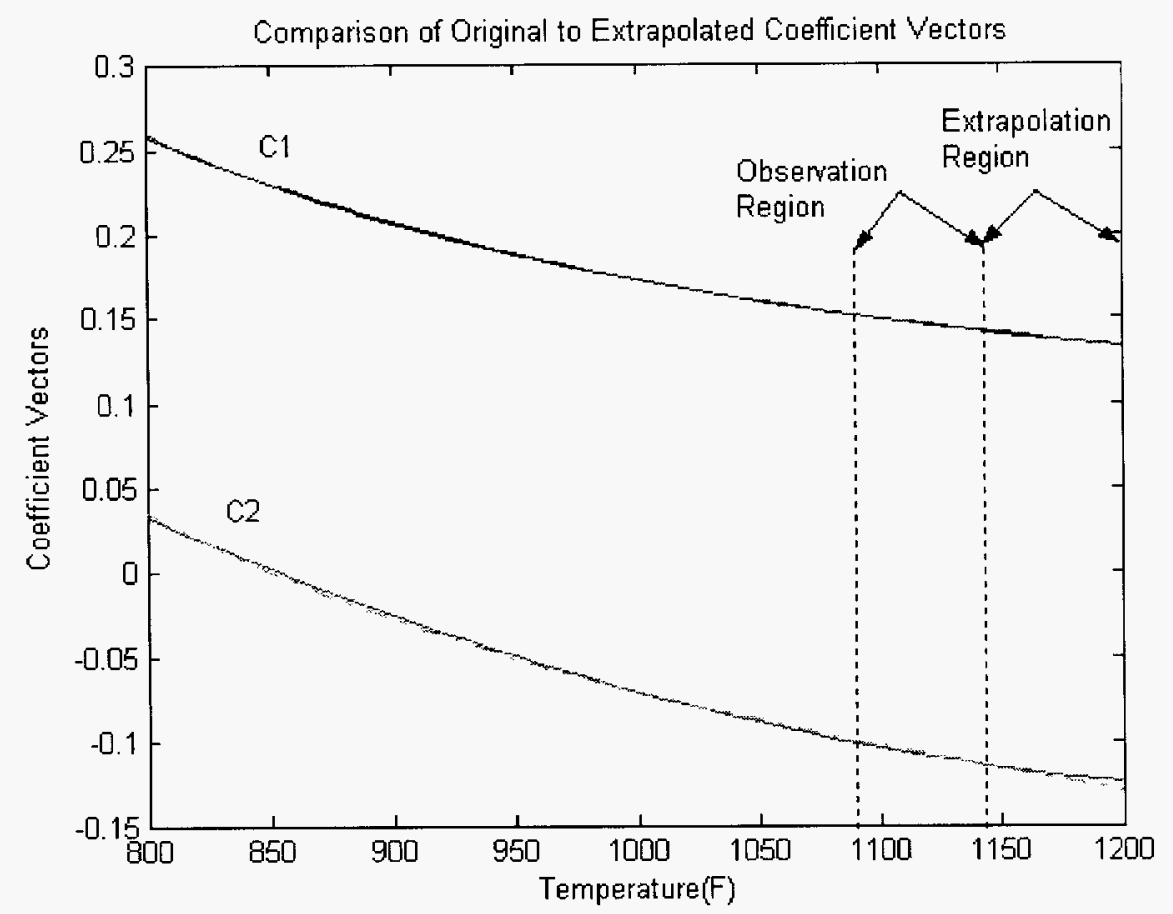

Fig. 3.20. Extrapolated coefficient vectors.

\subsubsection{Demonstration of Extrapolation Capability for Transient Heat Transfer}

Fig. 3.21 and Fig. 3.22 show the contour of the transient heat transfer at each $120 \mathrm{sec}, 130 \mathrm{sec}$, $180 \mathrm{sec}$ and $600 \mathrm{sec}$.
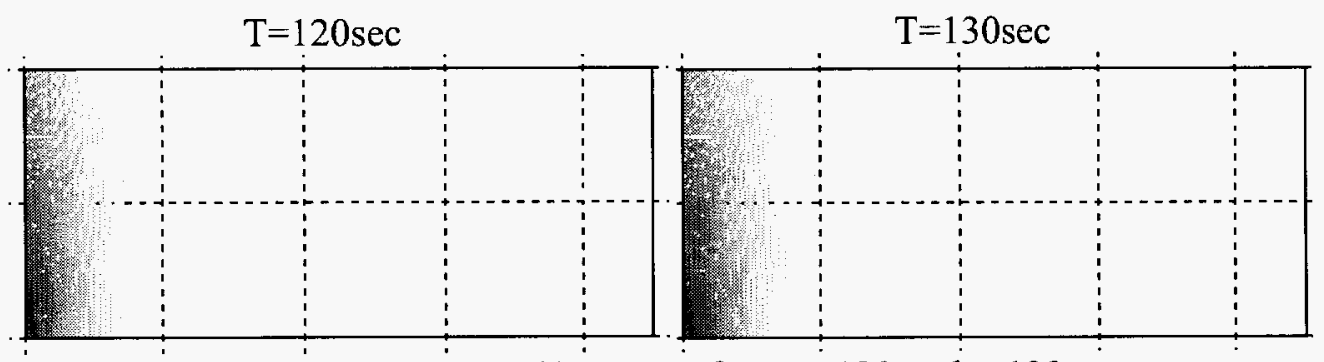

Fig. 3.21. Contour of heat transfer at $t=120$ and $t=130$ sec.

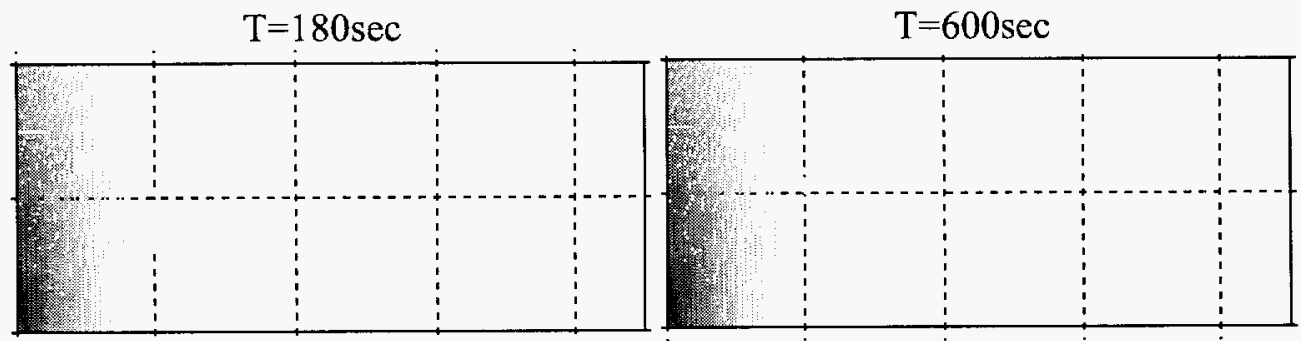

Fig. 3.22. Contour of heat transfer at $\mathrm{t}=180$ and $\mathrm{t}=600 \mathrm{sec}$. 
In this case, the basis set is chosen as $n=3$. Each basis set is arbitrary chosen by $115 \mathrm{sec}, 130 \mathrm{sec}$, and $150 \mathrm{sec}$. Fig. 3.23 shows the three rough coefficient.

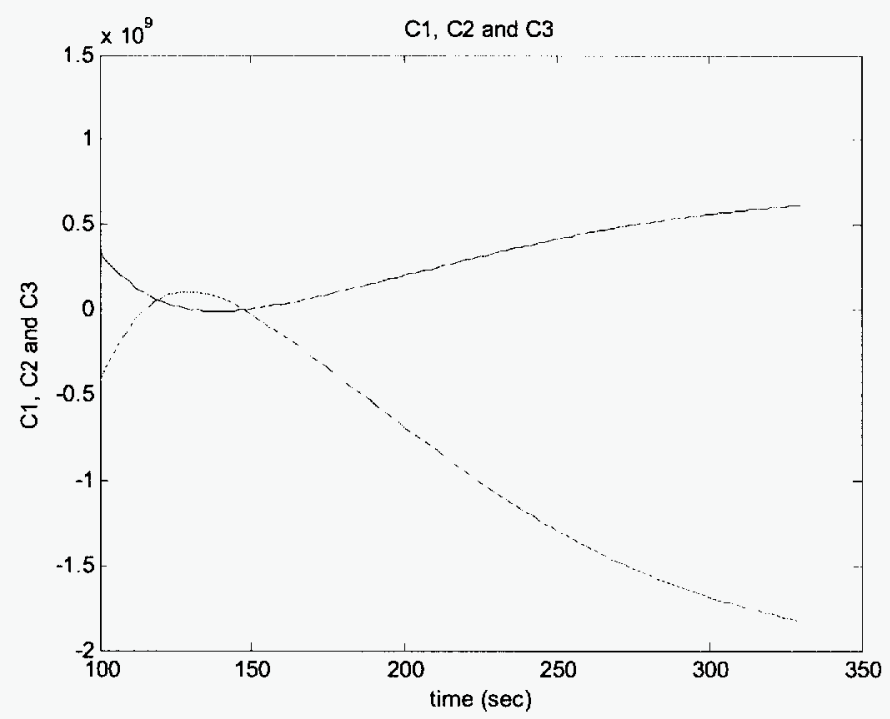

Fig. 3.23. Rough coefficient vectors.

Fig. 3.24 and Fig. 3.25 show the empirical temperature and computed temperature at $\mathrm{t}=248$ sec and Fig. 3. 26 show the error between two temperature profiles at $\mathrm{t}=248 \mathrm{sec}$.

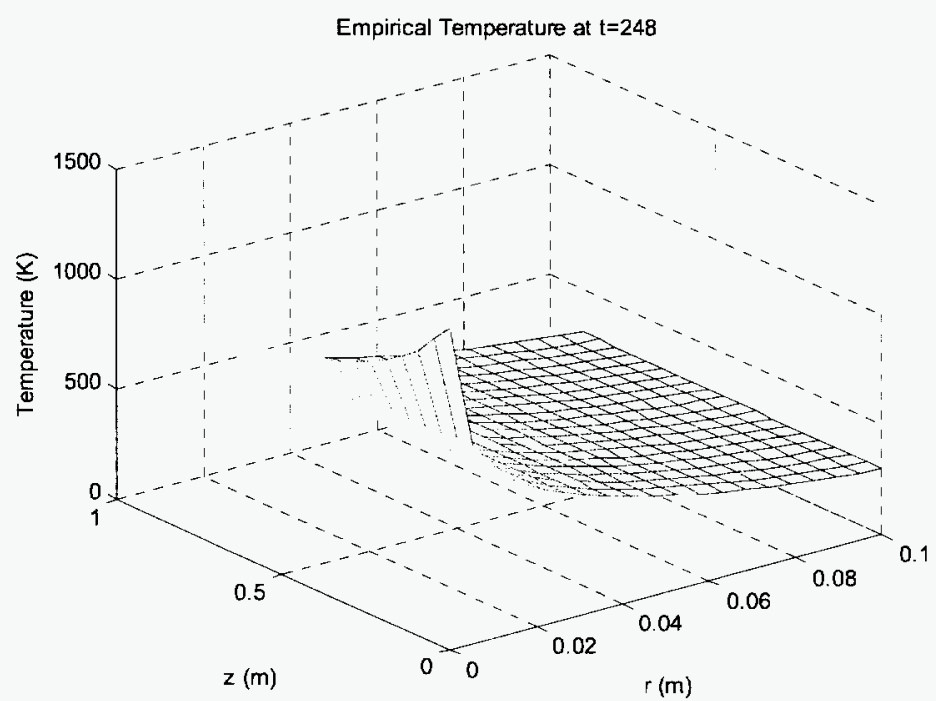

Fig. 3.24. Empirical temperature profile at $\mathrm{t}=248 \mathrm{sec}$. 


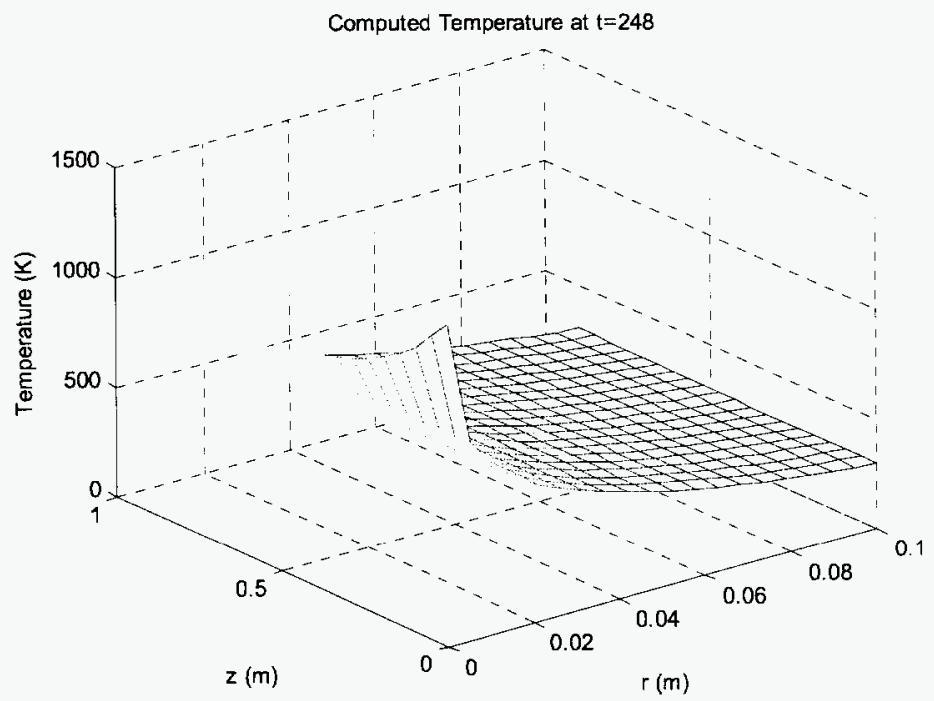

Fig. 3.25. Computed temperture profile at $t=248 \mathrm{sec}$.

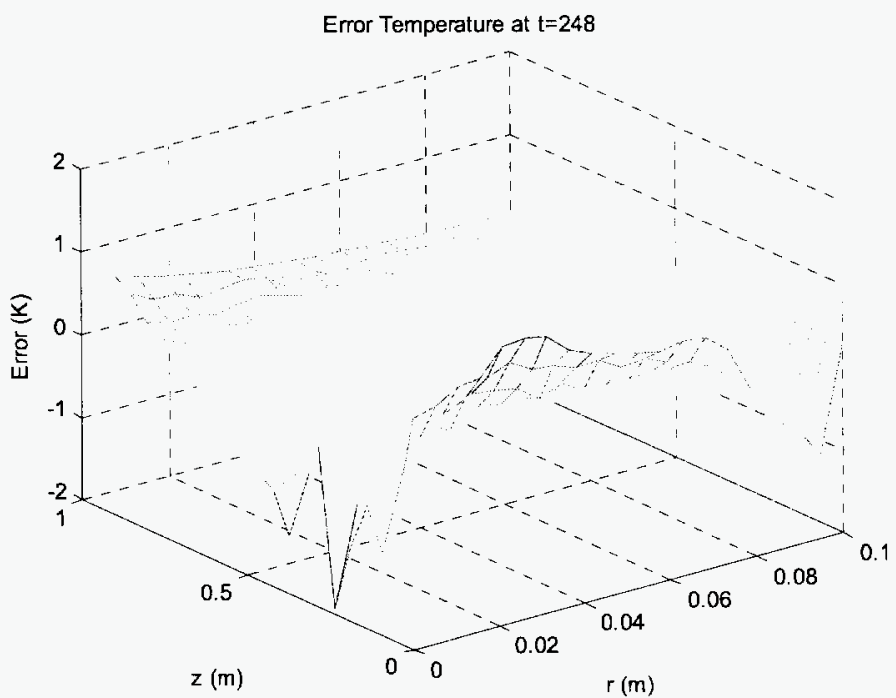

Fig. 3.26. Error between empirical and computed temperature at $t=248 \mathrm{sec}$.

Fig. 3.27 shows the smoothened coefficient vector $\mathrm{C} 1, \mathrm{C} 2$, and $\mathrm{C} 3$. 


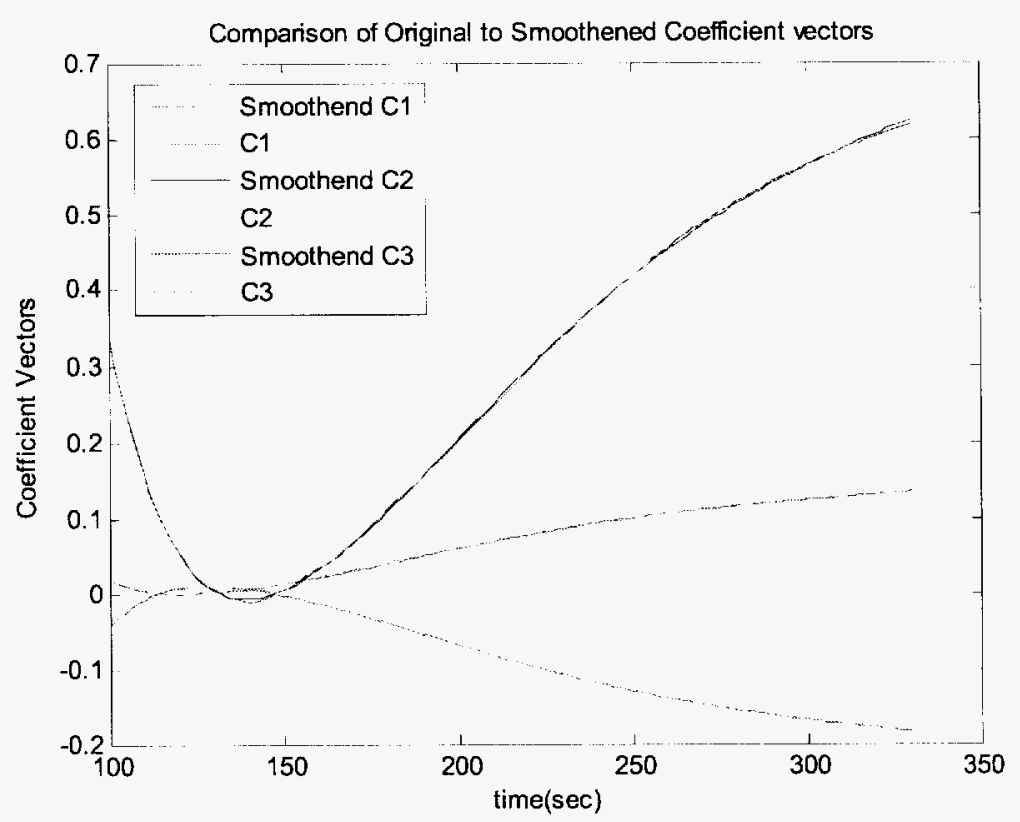

Fig. 3.27. Smoothened coefficient vector $\mathrm{Cl}$.

Fig. 3.28 shows the extrapolation test for each coefficient vector $\mathrm{C} 1, \mathrm{C} 2$, and $\mathrm{C} 3$. Extrapolation test is performed from $280 \mathrm{sec}$ to $300 \mathrm{sec}$ based on $260 \mathrm{sec}$ to $280 \mathrm{sec}$.

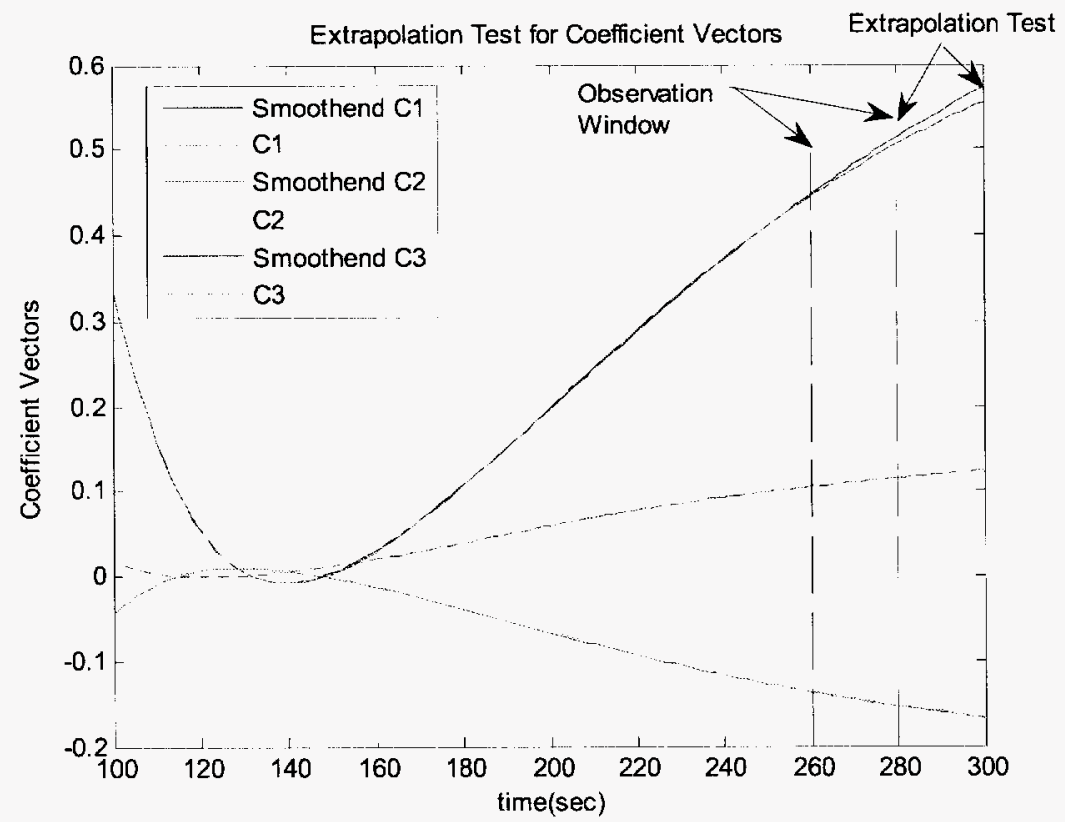

Fig. 3.28. Extrapolation test for $\mathrm{C} 1, \mathrm{C} 2$ and $\mathrm{C} 3$. 
Fig. 3.29 shows the extrapolation of the coefficient vectors from $300 \mathrm{sec}$ to $330 \mathrm{sec}$ based upon an observation window of 280 to $300 \mathrm{sec}$ which was previously used for the extrapolation test.

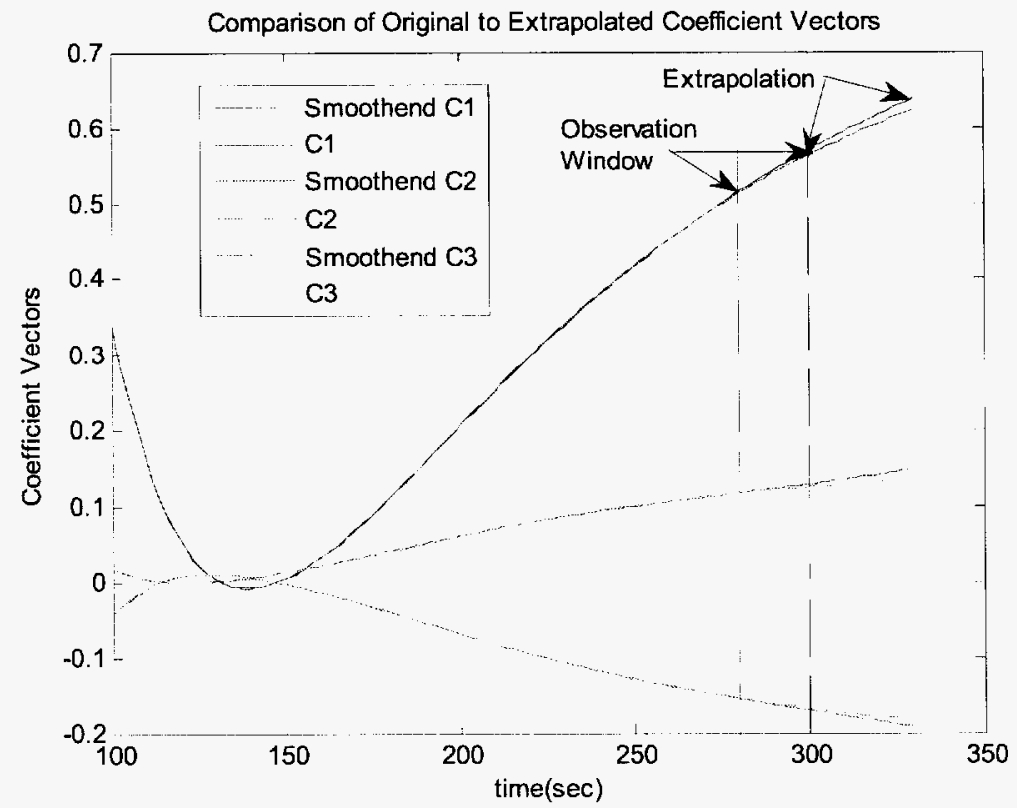

Fig. 3.29. Extrapolation for C1, C2, and C3.

\subsection{Conclusions}

In this research, we investigate a mathematical approach to extrapolation of the temperature distribution within a power plant boiler facility and enthalpy in a delivery section within a power plant, using a combination of a modified neural network architecture and semigroup theory. Given a set of empirical data with no analytic expression, we first develop an analytic description and then extend that model along a single axis. This can be achieved by using the algebraic decomposition to obtain an analytic description of empirical data in a specific form, called the semigroup form, which involves the product of a coefficient vector and a basis set of vectors. If this form can be achieved, the describing aspect is simplified because the description of the coefficient vector is decoupled from the description of the basis vector. Additionally, each component of the coefficient vector and each component of the basis set of vectors can be described individually. From the results, we conclude that the proposed system-type neural network architecture works well in temperature extrapolation, enthalpy extrapolation, and even transient heat transfer case. 


\subsection{References}

[26] K. Li and S. Thompson, "A cascaded neural network and its application to modeling power plant pollutant emission," Proceedings of the 3rd World Congress on Intelligent Control and Automation, pp. 992-997, Vol. 2, 28 June-2 July 2000.

[27] B. H. Kim, J. P. Velas, and K. Y. Lee, "Semigroup based neural network architecture for extrapolation of enthalpy in a power plant," Proceedings of the ISAP, pp. 291-296, 2005.

[28] K. Y. Lee, J. P. Velas, and B. H. Kim, "Development of an intelligent monitoring system with high temperature distributed fiberoptic sensor for fossil-fuel power plants," IEEE Power Engineering Society General Meeting, pp. 1350-1355, Jun 6-10, 2004.

[29] R Lanzafame and M. Messina, "A new method for the calculation of gases enthalpy," Energy Conversion Engineering Conference and Exhibit, vol.1, pp. 318 328, July 2000.

[30] M. A. Tahani and C. Lucas, "Development of expert controller for steam temperature regulation in power plants," IEEE/RSJ International Workshop on Intelligence for Mechanical Systems, vol.3, pp. 1333 - 1337, Nov. 1991.

[31] R. Jacobs and M. Jordan, "A competitive modular connectionist architecture," Advances in Neural Information Processing Systems, Vol. 3, pp. 767-773, Morgan-Kaufmann, Cal., 1991.

[32] A. Atiya, R. Aiyad, and S. Shaheen, "A practical gated expert system neural network," IEEE International Joint Conference on Neural Networks, Vol. 1, pp. 419-424, 1998.

[33] N. U. Ahmed, Semigroup theory with applications to systems and control, Longman Scientific \& Technical, Harlow, 1991.

[34] H. Tanabe, Equations of Evolution, Pitman Publishing Ltd., London, 1979.

[35] R. Padhi and S. N. Balakrishnan, "Proper orthogonal decomposition based feedback optimal control synthesis of distributed parameter systems using neural networks," Proceedings of the 2002 American Control Conference, 2002, pp. 4389-4394, Vol. 6, pp. 8-10, May 2002.

[36] S. Haykin, Neural Networks, $2^{\text {nd }}$ ed., Prentice Hall, N.J., 1999.

[37] M. Q. Phan and J. A. Frueh, "Learning control for trajectory tracking using basis functions," Proceedings of the 35th IEEE Conference on Decision and Control, pp. 24902492, Dec. 1996.

[38] A.N. Tikhonov, "On regularization of ill-posed problems," Doklady Akademii Nauk USSR, vol. 153, 1973.

[39] A.N. Tikhonov, "On solving incorrectly posed problems and method of regularization," Doklady Akademii Nauk vol. 151, USSR, 1973.

[40] C. C. Ku, K.Y. Lee, and R.M. Edwards, "Improved nuclear reactor temperature control using diagonal recurrent neural networks," IEEE Trans. on Nuclear Science, Vol. 39, pp. 2298-2308, December 1992.

[41] C. C. Ku and K.Y. Lee, "Diagonal recurrent neural networks for dynamic systems control," IEEE Trans. on Neural Networks, Vol. 6, pp. 144-156, January 1995.

[42] J. Elman., "Finding structure in time," Journal of Cognitive Science, Vol. 14, pp. 179-211, 1990.

[43] Miyadera, Nonlinear Semigroups, American Mathematical Society, Providence, R.I., 1992.

[44] Z. Altman and R. Mittra, "A technique for extrapolating numerically rigorous solutions of electromagnetic scattering problems to higher frequencies and their scaling properties," IEEE Transactions on Antennas and Propagation, Vol.: 47, No. 4, April 1999. 
[45] S. Richter and R. de Carlo, "Continuation methods: theory and applications," IEEE Transactions on Automatic Control, Vol. 28, pp. 660-665, 1983.

[46] NIST Chemistry WebBook. Available: http://webbook.nist.gov/chemistry/fluid/

[47] Y. A. Cengel, Heat Trasnfer: A Practical Approach, $2^{\text {nd }}$ edition, McGrawHill, N. Y., 2003.

[48] S. Moaveni, Finite Element Analysis: Theory and Application with ANSYS, Prentice Hall, N. J., 1999.

[49] M. Pedersen, Functional Analysis in Applied Mathematics and Engineering, Chapman \& Hall/CRC, N. Y., 1999.

[50] H. Tanabe, Equations of Evolution, Pitman Publishing Ltd., London, 1979. 


\section{CONCLUSIONS}

The objective of the proposed work is to develop an intelligent distributed fiber optical sensor system for real-time monitoring of high temperature in a boiler furnace in power plants. Of particular interest is the estimation of spatial and temporal distributions of high temperatures within a boiler furnace, which will be essential in assessing and controlling the mechanisms that form and remove pollutants at the source, such as NOx.

The basic approach in developing the proposed sensor system is three fold: (1) development of high temperature distributed fiber optical sensor capable of measuring temperatures greater than $2000 \mathrm{C}$ degree with spatial resolution of less than $1 \mathrm{~cm}$; (2) development of distributed parameter system (DPS) models to map the three-dimensional (3D) temperature distribution for the furnace; and (3) development of an intelligent monitoring system for real-time monitoring of the $3 \mathrm{D}$ boiler temperature distribution.

We have set up a dedicated high power, ultrafast laser system for fabricating in-fiber gratings in harsh environment optical fibers, successfully fabricated gratings in single crystal sapphire fibers by the high power laser system, and developed highly sensitive long period gratings (lpg) by electric arc. Relevant mathematical modeling studies of NOx formation in practical combustors have been completed. Studies show that in boiler systems with no swirl, the distributed temperature sensor may provide information sufficient to predict trends of NOx at the boiler exit. We have investigated a mathematical approach to extrapolation of the temperature distribution within a power plant boiler facility, using a combination of a modified neural network architecture and semigroup theory. Given a set of empirical data with no analytic expression, we first developed an analytic description and then extended that model along a single axis.

The developed Intelligent Monitoring System with High Temperature Distributed Fiberoptic Sensor can be effectively used for monitoring, not only the temperature distribution inside of the furnace, but also for controlling the combustion processes real-time in order to improve the fuel efficiency and reduce the pollutant to the environment. 


\section{TECHNICAL PUBLICATIONS}

[1] Chun Zhan, Jon Lee, Zhu Yong, and Shizhuo Yin, "Photo-enhanced polarization mode separated fiber Bragg gratings inscribed by femtosecond laser", Submitted to Jounal of Applied Physics.

[2] Chun Zhan, Yong Zhu and Shizhuo Yin, "Asymmetric Bragg gratings inscribed by IR femtosecond irradiation for harsh environment multi-parameter sensing applications", To be published in Optical Fiber Technology. (Invited paper)

[3] Zhu Yong, Chun Zhan, Jon Lee, and Shizhuo Yin, "Multiple parameter vector bending and high temperature sensors based on asymmetric multimode fiber Bragg gratings inscribed by infrared femtosecond laser" Opt. Lett. 31, 1794-1796 (2006).

[4] Y. Yang, K. Chung, S. Yin, Z. Liu, and Q. Wang, "Analysis of volume holographic long period grating in photonic nanostructured fibers and waveguides," Optical Engineering, Vol. 43, pp.2003-2008 (2004).

[5] D. Komisarek, K. Reichard, D. Merdes, D. Lysak, P.Lam, S. Wu, and S. Yin, "Highperformance nonscanning Fourier-transform spectrometer that uses a Wollaston prism array," Applied Optics, Vol. 43, pp.3983-3988, 2004.

[6] D. Komisarek, K. Reichard, and S. Yin, "Enhancing the performance of non-scanning Fourier transform spectrometer by compensating manufacturing defects inherent to a Wollaston prism array," Optics Communications, Volume 238, pp. 85-90 (2004).

[7] Bo Wang, Ruyan Guo, Shizhuo Yin and Francis Yu, "Chemical sensing with Hetero-Core Fiber Specklegram," Journal of Holography and Speckle 1, 53 (2004).

[8] K. Shi, P. Li, S. Yin, and Z. Liu, "Chromatic confocal microscopy using supercontinuum light," Optical Express, Vol. 12, No. 10, pp. 2096-2101 (2004).

[9] Kun-Wook Chung and Shizhuo Yin, "Analysis of a widely tunable long-period grating by use of an ultra thin cladding layer and higher-order cladding mode coupling," Optics Letters, Vol. 29, pp.812-814 (2004).

[10] Kun-wook Chung and Shizhuo Yin, "A highly nonlinear dispersion shifted fiber with 9.3 $\mathrm{mm} 2$ effective area and low loss for all fiber wavelength converter," Microwave and Optical Technology Letters," pp. 153-156 (2004).

\section{$\underline{\text { Refereed Conference Proceedings }}$}

[11] Chun Zhan, Yong Zhu, and Shizhuo Yin, "Fabricating harsh environment fiber Bragg gratings by ultrafast laser pulses", SPIE 6313-64, San Diego, Aug., 2006

[12] Stuart (Shizhuo) Yin, Sung-Hyun Nam, Yi Yang, Chun Zhan, and Kun-Wook Chung, "Innovative Fiber Optic Gratings: Fabrications and Applications," Proceedings of International Symposium on Advances and Trends in Fiber Optics and Applications, October 11-15, 2004, Chongqing University, Chongqing, China. (Invited Paper)

[13] S. Yin, C. Hahn*, J. Lee*, B. Wang*, and Q. Wang, „Design and implementation of an allfiber ultra-fast widely tunable wavelength filter (invited)," Proceedings of SPIE on Information Optics and Photonics Technology, SPIE, 5643, pp. *_* (2004) (Principal author; second, third, and fourth authors supervised by candidate) (Invited paper) 
[14] S. Nam, C. Zhun, S. Yin, "High temperature distributed fiber optic sensors," Proceeding s of SPIE on Fiber Optic Sensors, SPIE, 5634, pp. *-* (2004) (First and second authors supervised by candidate) (Invited paper)

[15] W. Su, Y. Hsu, C. Kuo, H. Chan, and S. Yin, "Design and fabrication of digital dualfreuqnecy patterns for projected fringe profilometry, SPIE 5606-20, Philadelphia, PA, Oct. 27, 2004.

[16] S. Yin, B. Wang, C. Luo, Q. Wang, K. Reichard, and D. Lysak, "Development of harsh environment electro-optic polymer and their applications to tunable photonic devices," IEEE LEOS Summer Topic meeting on Optical Interconnects \& VLSI Photonics, MB4.2, San Diego, CA, June 28, 2004.

[17] S. Nam, Chun Zhan, and S. Yin, "Recent advances on fabricating in-fiber gratings in single crystal sapphire fiber," SPIE 5560, pp.147-155, Denver, CO, Aug., 2004.

[18] Jon Lee, B. Wang, C. Hahn, K. Reichard, D. Ditto, D. Glista, Q. Wang, and S. Yin, "Harsh environment electro-optic polymer and its application to in-fiber tunable filter," SPIE 5560 , pp. 52-63, Denver, CO, Aug., 2004.

[19] B. Wang, Y. Yang, Y. Liu, S. Yin, R. Guo, P. Ruffin, "Fiber optic sensors using photonic crystal fiber embedded heterofiber structures," SPIE 5560, pp.284-294, Denver, CO, Aug., 2004.

[20] Wei-Hung Su, Cho-Yo Kuo, and Shizhuo Yin, "Design and fabrication of various digital fringe patterns for projected fringe profilometry," SPIE 5560, pp. 193-203, Denver, CO, Aug., 2004.

[21] K. Reichard, S. Yin, C. Hahn, J. Lee, B. Wang and Q. Wang, "An athermal design for an all-fiber, ultra fast, widely tunable wavelength filter," Avionics, Fiber-Optics and Photonics Workshop, Workshop Notes, ThP15, St. Louis Marriott Downtown, St. Louis, MO, 21-23 April 2004.

[22] S. Yin, S. Nam, P. Ruffin, K. Reichard, "An investigation of a photonic bandgap based gyroscope and its application to Avionics, Avionics, Fiber-Optics and Photonics Workshop, Workshop Notes, ThP17, St. Louis Marriott Downtown, St. Louis, MO, 21-23 April 2004.

[23] Sung-Hyun Nam, Jesus Chavez, and Shizhuo Yin, "Fabricating in fiber gratings in single crystal sapphire fibers," SPIE 5350, pp.58-65, San Jose, CA, Jan., 2004.

[24] Shizhuo Yin, Thomas W. Gardner*, Fei Wu and Milind Cholker, "Optical combing to align photoreceptors in detached retinas," SPIE 5314, pp.298-305, San Jose, CA, Jan. 2004.

[25] Yi Yang, KunWook Chung, Shizhuo Yin, Karl Reichard*, Zhiwen Liu, and Qing Wang+, "Highly sensitive sensors using in-fiber gratings fabricated in photonic crystal nanostructures," SPIE 5360, pp.237-246, San Jose, CA, Jan., 2004.

[26] B. H. Kim, J. P. Velas, and K. Y. Lee, "Semigroup based neural network architecture for extrapolation of enthalpy in a power plant," Proceedings of the ISAP, pp. 291-296, 2005.

[27] K. Y. Lee, J. P. Velas, and B. H. Kim, "Development of an intelligent monitoring system with high temperature distributed fiberoptic sensor for fossil-fuel power plants," IEEE Power Engineering Society General Meeting, pp. 1350-1355, Jun 6-10, 2004.

[28] Kim, B.-H., J. P. Velas, and K. Y. Lee, "Development of Intelligent Monitoring System for Fossil-Fuel Power Plants Using System Type Neural Networks and Semigroup Theory", Proc. of the IEEE Power Engineering Society General Meeting, in CD, San Francisco, June $12-17,2005$.

[29] Kim, B.-H., J. P. Velas, and K. Y. Lee, "Semigroup Based Neural Network Architecture for Extrapolation of Enthalpy in a Power Plant," Proc. International Conference on Intelligent 
System Application to Power Systems (ISAP 2005), pp. 291-296, Arlington, VA, USA, November $6-10,2005$.

[30] Kim, B.-H., J. P. Velas, and K. Y. Lee, "Semigroup based Neural Network Architecture for Extrapolation of Mass Unbalance for Rotating Machines in Power Plants," Proc. of the IFAC Symposium on Power Plants and Power Systems Control, in Kananaskis (near Calgary), Canada, June 25-28, 2006.

[31] Kim, B.-H., J. P. Velas, and K. Y. Lee, "Short-Term Load Forecasting Using System-Type Neural Network Architecture," Proc. of the IEEE World Congress on Computational Intelligence, pp. 4926-4933, Vancouver, Canada, July 16-21, 2006.

[32] Kim, B.-H., J. P. Velas, J.-K. Lee, J.-B. Park, J.-R. Shin, and K. Y. Lee, "Short-Term System Marginal Price Forecasting Using System-Type Neural Network Architecture," Proc. of the IEEE Power Systems Conference \& Exposition, Atlanta, GA, October 28November 3, 2006.

\section{ON-SITE VISIT}

We had an on-site visit from DOE on March 23, Wednesday, 2005. The following is the itinerary:

I. $8: 30$ am - 8:40 opening remarks

DOE: Susan Maley, Robert Romanosky, Peter Muchunas Hee Kim)

PSU: Kwang Lee, Stuart Yin, Andre Boehman, and Graduate Students (Mel Fox, Byong-

The Energy Institute, 405 Academic Activities Building

2. $8: 40$ - 9:30 am presentation

3. 9:30 - 10:00 lab tour at Energy Institute

4. 10:00 - 11:00 am travel to EE building and Lab tour at EE

Dr. Lee's Intelligent Distributed Controls Research Lab, 104 EE East

Dr. Yin's optics lab - In particular, harsh environment fiber optic grating fabrication facilities, EE East

5. 11:00 - 11:30 am, 129 EE East

Closing remarks.

\section{REVIEW MEETING}

We attended the annual review meeting held in June 2005 in Pittsburgh, PA. 